Universidade de São Paulo

Instituto de Física

\title{
Espalhamento Inelástico Profundo em Colisões Elétron-Íon
}

\author{
Erike Roberto Cazaroto
}

Orientador: Prof. Dr. Fernando Silveira Navarra

Dissertação de mestrado apresentada ao Instituto de Física para a obtenção do título de Mestre em Ciências

Comissão Examinadora:

Prof. Dr. Fernando Silveira Navarra (orientador) (IFUSP)

Prof. Dr. Alexandre Alarcon do Passo Suaide (IFUSP)

Prof. Dr. Francisco de Oliveira Durães (MACKEnZIE)

São Paulo

2009 


\section{FICHA CATALOGRÁFICA \\ Preparada pelo Serviço de Biblioteca e Informação \\ do Instituto de Física da Universidade de São Paulo}

Cazaroto, Érike Roberto

Espalhamento inelástico profundo em colisões elétroníon - São Paulo, 2009.

Dissertação (Mestrado) - Universidade de São Paulo. Instituto de Física. Departamento de Física Experimental.

Orientador: Prof. Dr. Fernando Silveira Navarra

Área de Concentração: Física

Unitermos: 1. Física de Partículas; 2.

Fenomenologia; 3. Glúons

USP/IF/SBI-079/2009 
À minha família,

À minha tia Creusa Maria Cazaroto, À minha avó Geni Valim Tofoli. 


\section{Agradecimentos}

- Agradeço ao professor Fernando Navarra, pela orientação e amizade.

- Agradeço à minha família pelo apoio ao longo de todos esses anos.

- Agradeço a todos professores, colegas do corredor e amigos do Grhafite: Marina Nielsen, Manoel Robillota e Celso Lima, Dunga, Sérgio, David, Gabriel, Ricardo, Jorgivan, Simone, Carina, Arthur, Pati, Dani, Babi, Celso, Raphael, pela companhia agradável de todos esses anos.

- Agradeço ao pessoal da secretaria, ao Paulo e ao Sérgio pelas ajudas nas dificuldades com o Latex e em vários outros problemas computacionais, e também pelos cafezinhos de toda tarde.

- Agradeço ao pessoal da Universidade Federal de Pelotas pela acolhida durante meu estágio com o professor Victor Gonçalves.

- Agradeço de modo geral a todos professores e colegas que contribuiram para minha formação ao longo de todos esses anos no Instituto de Física.

- Agradeço ao CNPq pelo apoio financeiro durante o mestrado. 


\section{Resumo}

Neste trabalho nós abordamos dois temas da QCD em altas energias. A distribuição nuclear de glúons e a saturação de pártons. As parametrizações da distribuição nuclear de glúons disponíveis atualmente na literatura são bastante diferentes entre si, o que mostra que esta distribuição ainda é bastante incerta. Nós mostramos que é possível vincular a distribuição de glúons no núcleo em altas energias a observáveis nucleares inclusivos, o que facilitará a distinção entre as diferentes parametrizações quando surgirem novos dados experimentais destes observáveis. O segundo tema abordado, a saturação de pártons, é uma questão muito importante atualmente, e a existência ou não deste fenômeno poderá ser definitivamente confirmada no LHC e no futuro eRHIC. No caso da saturação nós mostramos que os observáveis inclusivos não serão úteis para indicar se ocorre ou não a saturação de pártons em altas energias. Nossa conclusão foi a de que para os efeitos da saturação, ao invés destes observáveis devemos nos concentrar nos observáveis difrativos, que são muito mais sensíveis a dipolos de grande tamanho e corresponderão a uma fração significativa dos eventos observados. 


\section{Abstract}

In this work we considered two subjects of QCD at high energies. The nuclear gluon distributions and the parton saturation. The currently available parametrizations of nuclear gluon distribution in the literature are very different among themselves. We show that it is possible to relate the gluon distribution in a nucleus at high energies to nuclear inclusive observables. This will allow us to distinguish between the different parametrizations when new experimental data become available. The second subject considered, parton saturation, is currently very important, and the existence or not of this phenomenon will be confirmed at the LHC and at the future eRHIC. In the case of saturation we show that inclusive observables are not very useful to indicate if parton saturation occurs at high energies. Our conclusion was that to detect saturation effects, instead of these observables we must focus on the diffractive observables, which are much more sensitive to big dipoles and will correspond to a significant fraction of the observed events. 


\section{Sumário}

$\begin{array}{ll}\text { Introdução } & 1\end{array}$

1 Espalhamento inelástico profundo $\quad 3$

1.1 A cinemática do espalhamento inelástico profundo . . . . . . . . . . . . 3

1.2 O modelo a pártons . . . . . . . . . . . . . . . . . . . . . 9

1.3 As equações de DGLAP . . . . . . . . . . . . . . . . . . . 10

1.4 A equação de DGLAP no limite de pequeno $x \ldots \ldots$. . . . . . . . . 14

1.5 O DIS Nuclear . . . . . . . . . . . . . . . . . . . . 17

1.6 Efeito de "Shadowing" Nuclear . . . . . . . . . . . . . . . . . . . . . . . 18

1.7 Parametrizações das distribuições nucleares de partons ....... 19

1.8 Funções de estrutura longitudinal e de charme . . . . . . . . . . . . . . 22

1.9 Espalhamento difrativo . . . . . . . . . . . . . . . . . 23

2 Dipolos de cor $\quad 25$

2.1 DIS na representação de dipolos de cor . . . . . . . . . . . . . . . 25

2.1.1 Fatorização $k_{T}$ e a seção de choque de dipolo . . . . . . . . . . 27

2.2 O espalhamento difrativo no formalismo de dipolo de cor . . . . . . . . 30

3 Saturação de partons e as equações não lineares de evolução 34

3.1 Introdução . . . . . . . . . . . . . . . . . . . . . . . . 34

3.1.1 Evidências experimentais de saturação . . . . . . . . . . . . 39

3.2 A equação de Balitsky-Kovchegov . . . . . . . . . . . . . . . . . . . 40 
3.2.1 A equação de BK no limite de pequeno $x \ldots . . . . . . .44$

3.2.2 A equação de BK no limite de grandes dipolos . . . . . . . . . . 45

3.3 A equação de BFKL no limite de saturação . . . . . . . . . . . . . . . . 46

3.4 Modelos fenomenológicos . . . . . . . . . . . . . . . . 47

3.4 .1 O modelo de GBW . . . . . . . . . . . . . . 48

3.4.2 O modelo b-CGC . . . . . . . . . . . . . . . 49

4 Funções de estrutura e a distribuição nuclear de glúons 51

5 Efeitos de saturação em colisores $e A \quad 56$

5.1 A generalização nuclear da escala de saturação . . . . . . . . . . . . 56

5.2 Observáveis inclusivos nucleares . . . . . . . . . . . . . . 57

5.3 Previsões lineares e não-lineares . . . . . . . . . . . . . . . . . 59

5.3.1 Funções de estrutura inclusivas . . . . . . . . . . . . . . . . . . 59

5.3 .2 Seção de choque difrativa . . . . . . . . . . . . 65

$\begin{array}{llr}6 & \text { Conclusão } & 69\end{array}$

$\begin{array}{ll}\text { Apêndice } & 70\end{array}$

$\begin{array}{ll}\text { Apêndice } & 74\end{array}$

$\begin{array}{ll}\text { Referências Bibliográficas } & 76\end{array}$ 


\section{Lista de Figuras}

1.1 Espalhamento Inelástico Profundo. . . . . . . . . . . . . . . . . . . . 4

1.2 Espalhamento Inelástico Profundo no modelo a partons. . . . . . . . . 10

1.3 Função de estrutura $F_{2}$ (Extraído de [11]). . . . . . . . . . . . . . . . 12

1.4 Função de estrutura $F_{2}$ (Extraído de [11]). . . . . . . . . . . . . . . 13

1.5 Razão $R_{F_{2}}^{A}=F_{2}^{A} /\left(A F_{2}^{N}\right)$ (Extraído de [15]) . . . . . . . . . . . . 19

1.6 Razão $R_{g}=x g_{A} /\left(A x g_{N}\right)$ predita pelas parametrizações EKS, DS, HKN e EPS para $A=208$ e $Q^{2}=2,5 G e V^{2} \ldots \ldots \ldots 21$

3.1 Resultados de ZEUS para a distribuição de glúons. . . . . . . . . . . . . 35

3.2 Mapa da evolução da QCD. (Extraído de [4]). . . . . . . . . . . . . . . 37

3.3 Resolução em termos da virtualidade $Q^{2}$ para um fóton sondando o alvo. 38

3.4 As funções de estrutura $F_{2}$ (Extraído de [38]) . . . . . . . . . . . . . . . 41

3.5 Diagrama para a emissão de um glúon na evolução de um dipolo e seu limite de $N_{c} \rightarrow \infty$. . . . . . . . . . . . . . . . . . . . . 42

4.1 Razões $R_{g}, R_{C}$ e $R_{L}$, com $Q^{2}=2,5 G e V^{2}$ e $\mathrm{A}=208$, para as quatro parametrizações nucleares consideradas. . . . . . . . . . . . 52

4.2 Razões $R_{g}, R_{C}$ e $R_{L}$, com $Q^{2}=10 G e V^{2}$ e $\mathrm{A}=208$, para as quatro parametrizações nucleares consideradas. . . . . . . . . . . . 53

4.3 Razões $R_{g}, R_{C}$ e $R_{L}$, com $Q^{2}=1 G e V^{2}$ e $x=10^{-4}$, para as quatro parametrizações nucleares consideradas. . . . . . . . . . . . . . . . 54 
4.4 Razões $R_{g}, R_{C}$ e $R_{L}$, com $Q^{2}=10 G e V^{2}$ e $x=10^{-3}$, para as quatro parametrizações nucleares consideradas. . . . . . . . . . . . . 55

5.1 Escala de saturação para diferentes valores de $A$ e $x . \quad \ldots . . .57$

5.2 Razão $R_{F_{2}} \equiv 2 F_{2}^{A} / A F_{2}^{D}$ para $\mathrm{A}=\mathrm{Pb}$. Embora unidos por uma linha os resultados dos modelos de saturação foram calculados para $(\langle x\rangle$ ,$<Q^{2}>$ ) dos dados da colaboração E665 [50]. . . . . . . . . . . .

5.3 Razão $R_{F_{2}} \equiv 2 F_{2}^{A} / A F_{2}^{D}$ para $\mathrm{A}=$ Ca. Embora unidos por uma linha os resultados dos modelos de saturação foram calculados para $(<x\rangle$ ,$<Q^{2}>$ ) dos dados da colaboração E665 [50]. . . . . . . . . . . . .

5.4 Comparação entre as previsões para $R_{F_{2}}$ dos modelos de saturação (bCGC e GBW) e dos modelos de fatorização colinear (DS e EPS) para $Q^{2}=2,5 \mathrm{GeV}^{2}$ e $A=P b . \ldots \ldots \ldots 6$

5.5 Comparação entre as previsões para $R_{F_{2}}$ dos modelos de saturação (bCGC e GBW) e dos modelos de fatorização colinear (DS e EPS) para $Q^{2}=10 G_{e V}^{2}$ e $A=P b \ldots \ldots \ldots \ldots$

5.6 Comparação entre as previsões para $R_{L}=2 F_{L}^{A} / A F_{L}^{D}$ dos modelos de saturação (b-CGC e GBW) e dos modelos de fatorização colinear (DS e EPS) para $Q^{2}=2,5 G e V^{2}$ e $A=P b \ldots \ldots \ldots$

5.7 Comparação entre as previsões para $R_{L}=2 F_{L}^{A} / A F_{L}^{D}$ dos modelos de saturação (b-CGC e GBW) e dos modelos de fatorização colinear (DS e EPS) para $Q^{2}=10 \mathrm{GeV}^{2}$ e $A=P b \ldots \ldots \ldots$

5.8 Comparação entre as previsões para $R_{C}=2 F_{2}^{c, A} / A F_{2}^{c, D}$ dos modelos de saturação (b-CGC e GBW) e dos modelos de fatorização colinear (DS e EPS) para $Q^{2}=2,5 G e V^{2}$ e $A=P b \ldots \ldots \ldots$

5.9 Comparação entre as previsões para $R_{C}=2 F_{2}^{c, A} / A F_{2}^{c, D}$ dos modelos de saturação (b-CGC e GBW) e dos modelos de fatorização colinear (DS e EPS) para $Q^{2}=10 G_{e V}^{2}$ e $A=P b \ldots \ldots \ldots$ 
5.10 Razão $R_{\sigma}=\sigma_{d i f f} / \sigma_{t o t}$, como função de $x$, com o modelo b-CGC para $\mathrm{A}=\mathrm{Pb}$ e $\mathrm{A}=$ Ca e para $Q^{2}=5 G e V^{2}$ e $Q^{2}=1 G e V^{2} \ldots \ldots 7$

5.11 Razão $R_{\sigma}=\sigma_{d i f f} / \sigma_{t o t}$, como função de $x$, com o modelo GBW para A $=\mathrm{Pb}$ e $\mathrm{A}=$ Ca e para $Q^{2}=5 G e V^{2}$ e $Q^{2}=1 G e V^{2} \ldots \ldots 68$ 


\section{Introdução}

Um dos principais objetivos da física hadrônica de altas energias atualmente é procurar sinais de saturação de pártons. Várias evidências deste fenômeno já foram apontadas na literatura por diversos autores. Entretanto, existem também trabalhos mostrando que o mesmo conjunto de dados pode ser descrito sem levar em conta estes efeitos. O Large Hadron Collider (LHC) e o futuro eRHIC poderão nos próximos anos confirmar se realmente ocorre saturação.

Nesta dissertação apresentamos dois trabalhos que realizamos ao longo do meu mestrado. Um destes trabalhos está relacionado à física linear, que é a física que não leva em conta efeitos de saturação. Os resultados deste trabalho foram publicados na referência [1] e são apresentados no capítulo 4 desta dissertação. Nele nós comparamos quatro parametrizações disponíveis na literatura para as distribuições nucleares de glúons e verificamos que elas são muito diferentes umas das outras, especialmente na região de altas energias. Tendo em vista a importância da distribuição de glúons nos cálculos das seções de choque em colisões a altas energias, uma determinação precisa de sua distribuição é fundamental. Nós verificamos a possibilidade de estabelecer vínculos para a distribuição de glúons em um núcleo usando observáveis nucleares inclusivos que serão medidos nos futuros colisores $e A$.

Nosso outro trabalho foi relacionado à física de saturação. Os resultados obtidos foram publicados na referência [2] e são apresentados no capítulo 5. Tendo em vista que a questão sobre a existência ou não de efeitos de saturação ainda não foi respondida, nós analisamos a possibilidade de identificar estes efeitos nos futuros experimentos de 
colisões $e A$ olhando para os observáveis inclusivos.

Em resumo, os resultados originais estão todos nos capítulos 4 e 5 . Os primeiros três capítulos apresentam uma descrição dos conceitos envolvidos nestes trabalhos. O material contido nestes três capítulos foi extraído de teses, livros e artigos, sendo as principais fontes as teses de doutorado [3] e [4] e o livro [5].

No capítulo 1 há uma descrição do espalhamento inelástico profundo (DIS) e uma apresentação das funções de estrutura, que são quantidades observáveis num experimento de DIS. Apresentamos também o modelo à pártons, que é equivalente ao diagrama de ordem zero da QCD, e as equações de evolução de DGLAP, que representam as correções a esse diagrama.

No capítulo 2 apresentamos o formalismo de dipolo de cor e fazemos um esboço de como o formalismo de fatorização $k_{T}$ permite escrever a seção de choque fóton virtual - núcleo como uma convolução da seção de choque dipolo - núcleo com a função de onda de transição fóton virtual $\rightarrow$ dipolo. Finalmente, esboçamos uma derivação da seção de choque difrativa no formalismo da mecânica quântica.

No capítulo 3 apresentamos uma discussão do conceito de saturação de pártons, assim como das equações de evolução obtidas nos modelos de saturação. Apresentamos também os modelos fenomenológicos que são utilizados no capítulo 5 .

Nesta dissertação optamos por deixar alguns termos e siglas em inglês, como por exemplo: scaling, splitting, slope, CGC, QCD, etc. 


\section{Capítulo 1}

\section{Espalhamento inelástico profundo}

\subsection{A cinemática do espalhamento inelástico profundo}

O espalhamento inelástico profundo (Deep Inelastic Scattering - DIS) é capaz de fornecer um importante teste para a cromodinâmica quântica perturbativa (pQCD). Além disso ele é o modo mais direto de explorar a estrutura interna dos hádrons.

Vamos descrever à seguir a cinemática do processo de espalhamento lépton-hádron, que é o mais importante para esta dissertação. Neste processo um feixe de léptons bombardeia um alvo hadrônico, que pode ser um nucleon $N$ ou um núcleo $A$, e sofre espalhamento inelástico [3]

$$
l+N \rightarrow l+X
$$

onde $l$ representa o lépton, $N$ o nucleon e $X$ o sistema de hádrons produzidos no processo inelástico. Experimentos nos quais não identificamos os hádrons produzidos $X \mathrm{e}$ medimos apenas o momento do lépton no estado final são chamados de experimentos inclusivos. Quando conseguimos identificar todos os hádrons produzidos no estado final temos os chamados experimentos exclusivos. Na figura 1.1 temos o diagrama de Feynman para o processo descrito em $(1,1.1)$.

O estudo de experimentos inclusivos requer três variáveis cinemáticas indepen- 


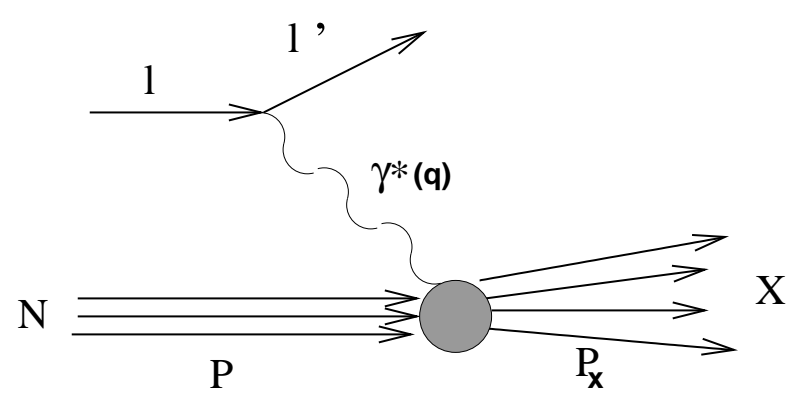

Figura 1.1: Espalhamento Inelástico Profundo.

dentes. Podemos escolher, por exemplo:

$$
s=(l+P)^{2}, \quad q^{2} \equiv-Q^{2}=\left(l-l^{\prime}\right)^{2}, \quad W^{2}=(P+q)^{2},
$$

onde $l$ e $l^{\prime}$ são, respectivamente, os quadri-momentos inicial e final do lépton; $P$ é o quadri-momento do nucleon; $q^{2}$ é o quadri-momento quadrado transferido do lépton através do fóton virtual emitido e $Q^{2}$ é chamada de "virtualidade do fóton". A variável $s$ corresponde ao quadrado da energia total do sistema lépton-nucleon no referencial do centro de massa e $W^{2}$ é o quadrado da massa invariante do estado hadrônico final $X$.

No referencial de repouso do alvo (referencial do laboratório) temos $P=\left(m_{N}, 0,0,0\right)$, onde $m_{N}$ é a massa do nucleon, e as variáveis $s, q^{2}$ e $W^{2}$ podem ser reescritas da seguinte forma:

$$
\begin{aligned}
s & =m_{N}\left(2 E+m_{N}\right), \\
q^{2} & =-4 E^{\prime} \operatorname{sen}^{2}(\theta / 2), \\
W^{2} & =m_{N}^{2}+2 m_{N}\left(E-E^{\prime}\right)+q^{2},
\end{aligned}
$$

onde a massa do lépton foi desprezada, $\theta$ é o ângulo de espalhamento do lépton no referencial do laboratório e $\nu=\left(E-E^{\prime}\right)$ é a energia transferida. Frequentemente 
define-se a variável $\nu$ como: $\nu=\frac{P . q}{m_{N}}$. Outras variáveis que surgem no DIS são:

$$
\begin{aligned}
& y=\frac{P . q}{P . l}=\frac{W^{2}+Q^{2}-m_{N}^{2}}{s-m_{N}^{2}} \\
& x=\frac{Q^{2}}{2 P . q}=\frac{Q^{2}}{2 m_{N} \nu}=\frac{Q^{2}}{W^{2}+Q^{2}-m_{N}^{2}}
\end{aligned}
$$

onde $y$ representa a fração da energia inicial do lépton carregada pelo fóton trocado, $y=\nu / E$; e $x$ é a variável de Bjorken, que será bastante utilizada no decorrer dessa dissertação, especialmente quando considerarmos a região em que essa variável é muito pequena. Uma relação útil entre $x, y$ e $Q^{2}$ é dada por:

$$
x y=\frac{Q^{2}}{s-m_{N}^{2}} \approx \frac{Q^{2}}{s} .
$$

Como $W^{2} \geq m_{N}^{2}$, a variável de Bjorken $(x)$ e $y$ assumem valores entre $0<x, y<1$. A expressão "deep inelastic" vem do regime cinemático onde ambos $m_{N} \nu$ e $Q^{2}$ são muito maiores do que $m_{N}^{2}$, $\operatorname{com} x$ fixo e finito.

A seção de choque do processo descrito acima (considerando-o não polarizado) é $[5]:$

$$
d \sigma=\frac{1}{4(l . P)} \frac{1}{2} \sum_{s_{l}, s_{l^{\prime}}} \frac{1}{2} \sum_{S} \sum_{X} \int \frac{d^{3} P_{X}}{(2 \pi)^{3} 2 P_{X}^{0}}(2 \pi)^{4} \delta^{4}\left(P+l-P_{X}-l^{\prime}\right)|M|^{2} \frac{d^{3} l^{\prime}}{(2 \pi)^{3} 2 E^{\prime}},
$$

onde o quadrado da amplitude é dado por:

$$
|M|^{2}=\frac{e^{4}}{q^{4}}\left[\bar{u}_{l^{\prime}}\left(l^{\prime}, s_{l^{\prime}}\right) \gamma_{\mu} u_{l}\left(l, s_{l}\right)\right]^{*}\left[\bar{u}_{l^{\prime}}\left(l^{\prime}, s_{l^{\prime}}\right) \gamma_{\nu} u_{l}\left(l, s_{l}\right)\right]\left\langle X\left|J^{\mu}(0)\right| P, S\right\rangle^{*}\left\langle X\left|J^{\nu}(0)\right| P, S\right\rangle
$$

Aqui podemos introduzir o tensor hadrônico $W^{\mu \nu}$ :

$$
\begin{aligned}
W^{\mu \nu} & \equiv \frac{1}{(2 \pi)} \frac{1}{2} \sum_{S} \sum_{X} \int \frac{d^{3} P_{X}}{(2 \pi)^{3} 2 P_{X}^{0}}(2 \pi)^{4} \delta^{4}\left(P+q-P_{X}\right)\left\langle P, S\left|J^{\mu}(0)\right| X\right\rangle\left\langle X\left|J^{\nu}(0)\right| P, S\right\rangle \\
& =\frac{1}{2 \pi} \int d^{4} z e^{i q . z}\left\langle N\left|J^{\mu}(z) J^{\nu}(0)\right| N\right\rangle
\end{aligned}
$$


onde $J_{\mu}$ é a corrente de transição hadrônica; e desprezando as massas dos léptons, o tensor leptônico $L_{\mu \nu}$ é definido como:

$$
\begin{aligned}
L_{\mu \nu} & \equiv \frac{1}{2} \sum_{s_{l}, s_{l^{\prime}}}\left[\bar{u}_{l^{\prime}}\left(l^{\prime}, s_{l^{\prime}}\right) \gamma_{\mu} u_{l}\left(l, s_{l}\right)\right]^{*}\left[\bar{u}_{l^{\prime}}\left(l^{\prime}, s_{l^{\prime}}\right) \gamma_{\nu} u_{l}\left(l, s_{l}\right)\right] \\
& =\frac{1}{2} \operatorname{Tr}\left[\gamma_{\mu} \gamma^{\prime} \gamma_{\nu}\right] \\
& =2\left(l_{\mu} l_{\nu}^{\prime}+l_{\nu} l_{\mu}^{\prime}-g_{\mu \nu} l . l^{\prime}\right)
\end{aligned}
$$

Substituindo $(1,1.8)$ e $(1,1.9)$ em $(1,1.6)$ obtemos:

$$
\frac{d^{2} \sigma}{d E^{\prime} d \Omega}=\frac{\alpha_{e m}^{2}}{2 m_{N} Q^{4}} \frac{E^{\prime}}{E} L_{\mu \nu} W^{\mu \nu}
$$

onde $d \Omega$ é o ângulo sólido que identifica a direção do lépton que sai (l'):

$$
d \Omega=d \cos \theta d \phi
$$

e

$$
\alpha_{e m}=\frac{e^{2}}{4 \pi} .
$$

O tensor hadrônico $W^{\mu \nu}$ pode ser parametrizado em termos de $g^{\mu \nu}, P^{\mu}$ e $q^{\mu}$ presentes no vértice hadrônico, e com isto podemos escrever $W^{\mu \nu}$ da seguinte maneira [5]:

$$
\begin{aligned}
\frac{1}{2 m_{N}} W^{\mu \nu}=\left[-g^{\mu \nu}+\right. & \left.\frac{q^{\mu} q^{\nu}}{q^{2}}\right] W_{1}\left(P . q, q^{2}\right) \\
& +\frac{1}{m_{N}^{2}}\left[\left(P^{\mu}-\frac{P . q}{q^{2}} q^{\mu}\right)\left(P^{\nu}-\frac{P . q}{q^{2}} q^{\nu}\right)\right] W_{2}\left(P . q, q^{2}\right) .
\end{aligned}
$$

onde $W_{1}$ e $W_{2}$ são funções de estrutura inelásticas. Assim escrevemos o produto $L_{\mu \nu} W^{\mu \nu}$, que aparece na equação $(1,1.10)$, no referencial do laboratório como:

$$
L_{\mu \nu} W^{\mu \nu}=4 E E^{\prime}\left[2 W_{1} \operatorname{sen}^{2} \frac{\theta}{2}+W_{2} \cos ^{2} \frac{\theta}{2}\right] 2 m_{N}
$$


Substituindo $(1,1.12)$ na expressão $(1,1.10)$ chegamos em:

$$
\frac{d^{2} \sigma}{d E^{\prime} d \Omega}=\frac{4 \alpha_{e m}^{2} E^{\prime 2}}{Q^{4}}\left[2 W_{1} \operatorname{sen}^{2} \frac{\theta}{2}+W_{2} \cos ^{2} \frac{\theta}{2}\right]
$$

A seção de choque diferencial pode ser reescrita em termos das variáveis do DIS

$$
\begin{aligned}
\left.\frac{d \sigma}{d y d x}\right|_{l a b}= & \frac{2 \pi m_{N} E y}{E^{\prime}} \frac{\alpha_{e m}^{2}}{4 E^{2}\left(\frac{x y m_{N}}{2 E^{\prime}}\right)^{2}} \\
& \times\left[2 W_{1} \frac{x y m_{N}}{2 E^{\prime}}+W_{2} \frac{E}{E^{\prime}}\left(1-y-\frac{m_{N} x y}{2 E}\right)\right] \\
= & \frac{2 \pi \alpha_{e m}^{2}}{E m_{N} x^{2} y}\left[x y m_{N} W_{1}+E\left(1-y-\frac{m_{N} x y}{2 E}\right) W_{2}\right] \\
= & \frac{2 \pi \alpha_{e m}^{2}}{E m_{N} x^{2} y^{2}}\left[x y^{2} F_{1}+\left(1-y-\frac{m_{N} x y}{2 E}\right) F_{2}\right]
\end{aligned}
$$

onde definimos as funções de estrutura adimensionais:

$$
F_{1}\left(x, Q^{2}\right) \equiv m_{N} W_{1}\left(\nu, Q^{2}\right)
$$

e

$$
F_{2}\left(x, Q^{2}\right) \equiv \nu W_{2}\left(\nu, Q^{2}\right)
$$

Além disso, como $y=\frac{Q^{2}}{2 m_{N} x E}$, podemos escrever

$$
d y=\frac{d Q^{2}}{2 m_{N} x E}
$$

e também

$$
x^{2}=\frac{Q^{4}}{4 m_{N}^{2} E^{2} y^{2}} .
$$

Portanto,

$$
\left.\frac{d \sigma}{d x d Q^{2}}\right|_{l a b}=\frac{4 \pi \alpha_{e m}^{2}}{x Q^{4}}\left[x y^{2} F_{1}+\left(1-y-\frac{x y m_{N}}{2 E}\right) F_{2}\right] .
$$


Quando $\sqrt{s} \gg m_{N}$ :

$$
\left.\frac{d \sigma}{d x d Q^{2}}\right|_{l a b} \approx \frac{4 \pi \alpha_{e m}^{2}}{x Q^{4}}\left[x y^{2} F_{1}+(1-y) F_{2}\right]
$$

As funções de estrutura descrevem a estrutura do nucleon medida no espalhamento inelástico profundo inclusivo. As funções de estrutura do DIS podem ser relacionadas, via teorema ótico, com a seção de choque total de absorção do fóton virtual $[5,6]$ :

$$
\sigma_{\lambda}^{\gamma^{*} N}\left(x, Q^{2}\right)=\frac{2 \pi^{2} \alpha_{e m}}{m_{N} \sqrt{\nu^{2}+Q^{2}}} \varepsilon_{\mu}^{(\lambda)} \varepsilon_{\nu}^{(\lambda)^{*}} W^{\mu \nu},
$$

onde $\varepsilon_{\mu}^{(\lambda)}$ é a polarização quadri-vetorial de um fóton virtual de helicidade $\lambda$. Em termos de $F_{1}$ e $F_{2}$ as seções de choque transversal e longitudinal são dadas respectivamente por:

$$
\sigma_{T}^{\gamma^{*} N}=\frac{4 \pi^{2} \alpha_{e m}}{Q^{2}} 2 x F_{1}
$$

$\mathrm{e}$

$$
\sigma_{L}^{\gamma^{*} N}=\frac{4 \pi^{2} \alpha_{e m}}{Q^{2}}\left(F_{2}-2 x F_{1}\right)
$$

E agora definindo:

$$
F_{T}=2 x F_{1}
$$

e

$$
F_{L}=F_{2}-2 x F_{1}
$$

temos:

$$
\sigma_{L, T}^{\gamma^{*} N}=\frac{4 \pi^{2} \alpha_{e m}}{Q^{2}} F_{L, T}\left(x, Q^{2}\right)
$$

Observe que: $F_{L}+F_{T}=F_{2}$. Assim obtemos a relação da seção de choque total com $F_{2}$ :

$$
\sigma^{\gamma^{*} N}=\sigma_{L}^{\gamma^{*} N}+\sigma_{T}^{\gamma^{*} N}=\frac{4 \pi^{2} \alpha_{e m}}{Q^{2}} F_{2}\left(x, Q^{2}\right)
$$




\subsection{O modelo a pártons}

Em 1969 Bjorken mostrou que se o nucleon fosse feito de férmions puntiformes e não interagentes, quando: $\nu, Q^{2} \rightarrow \infty, F_{1}$ e $F_{2}$ deveriam ser funções apenas de uma variável $x=\frac{Q^{2}}{2 m_{N} \nu}$, e não mais de $\nu$ e $Q^{2}$ separadamente. Assim as funções de estrutura deveriam exibir o chamado scaling de Bjorken [3, 7]:

$$
F_{i}\left(x, Q^{2}\right) \approx F_{i}(x), \quad i=1,2
$$

Como será discutido mais adiante, essa relação é válida apenas no modelo a pártons não interagentes. Em QCD existe uma dependência suave em $Q^{2}$, de tipo logarítmica, e por isto essa relação é violada. É a chamada violação do scaling de Bjorken.

Ainda em 1969 dados experimentais do SLAC confirmaram as previsões de Bjorken. Esse resultado foi interpretado [8,9] como uma evidência de que o próton é composto por partículas elementares (puntiformes): os pártons. Nesta interpretação o fóton virtual $\left(\gamma^{*}\right)$ é espalhado incoerentemente com os constituintes internos do nucleon.

A hipótese de espalhamento incoerente implica que a seção de choque total $\gamma^{*}$ próton é igual à soma das seções de choque $\gamma^{*}$-párton, pesada pela probabilidade $f_{i}(\xi)$ de encontrar um párton $q_{i}$ dentro do próton carregando uma fração $\xi$ de seu quadri-momento:

$$
\frac{d^{2} \sigma}{d x d Q^{2}}=\sum_{i} \int_{0}^{1} d \xi f_{i}(\xi) \frac{d^{2} \hat{\sigma}}{d x d Q^{2}}
$$

Deste modo introduzimos a "distribuição de partons" do tipo $i, f_{i}(\xi)$, dentro do nucleon. A expressão $(1,2.23)$ reflete a fatorização da seção de choque do DIS em uma interação de curta distância, descrita pela seção de choque partônica $d \hat{\sigma}(\xi)$, e uma função que contém os efeitos de longa distância, a distribuição de partons $f(\xi)$.

Assumindo que os partons sejam férmions de Dirac com spin 1/2, carregando uma fração $\xi$ do momento do próton, o seguinte resultado é encontrado [10]:

$$
F_{2}(x)=2 x F_{1}(x)=\sum_{i} e_{i}^{2} \int_{0}^{1} d \xi \delta(x-\xi) \xi f_{i}(\xi)=\sum_{i} e_{i}^{2} x f_{i}(x)
$$




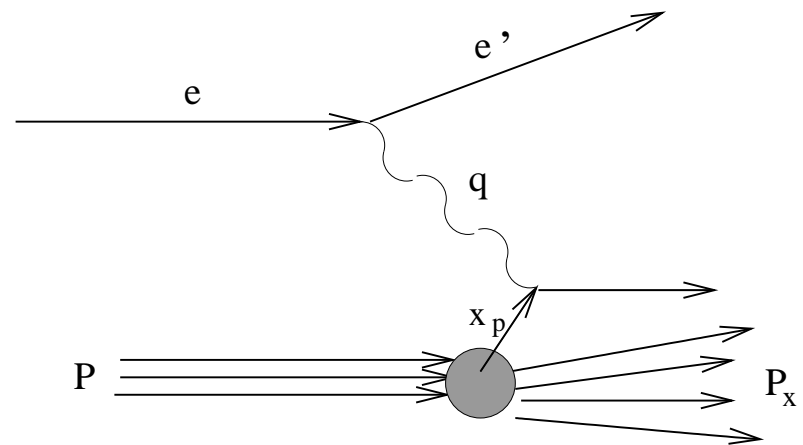

Figura 1.2: Espalhamento Inelástico Profundo no modelo a partons.

onde $e_{i}$ é a carga elétrica do quark de sabor $i$. A relação de Callan-Gross, $F_{2}(x)=$ $2 x F_{1}(x)$, que implica que a seção de choque longitudinal é zero, vem da hipótese de spin igual a 1/2. Além disso, o $x$ de Bjorken é igual à fração de momento do nucleon carregada pelo parton que colide. Vemos assim que a função de estrutura $F_{2}(x)$ mede a distribuição de partons dentro do próton.

\subsection{As equações de DGLAP}

As equações de Dokshitzer-Gribov-Lipatov-Altarelli-Parisi (DGLAP) descrevem a influência das correções perturbativas da QCD nas funções de distribuição que entram no modelo de partons dos processos de espalhamento inelástico profundo [3]. Elas descrevem a evolução, na variável $Q^{2}$, das funções de distribuição dos partons, e dão uma medida da violação de scaling (de Bjorken) da função de estrutura $F_{2}$.

A equação de DGLAP para a distribuição de um quark $q_{i}$, de sabor $i$, é $[6,4]$ :

$$
\frac{\partial q_{i}\left(x, Q^{2}\right)}{\partial \ln Q^{2}}=\frac{\alpha_{s}}{2 \pi}\left[\int_{x}^{1} \frac{d x_{1}}{x_{1}}\left(P_{q q}\left(\frac{x}{x_{1}}\right) q_{i}\left(x_{1}, Q^{2}\right)+P_{q g}\left(\frac{x}{x_{1}}\right) g\left(x_{1}, Q^{2}\right)\right)\right],
$$

e para a distribuição de glúons temos:

$$
\frac{\partial g\left(x, Q^{2}\right)}{\partial \ln Q^{2}}=\frac{\alpha_{s}}{2 \pi}\left[\int_{x}^{1} \frac{d x_{1}}{x_{1}}\left(P_{g q}\left(\frac{x}{x_{1}}\right) q_{S}\left(x_{1}, Q^{2}\right)+P_{g g}\left(\frac{x}{x_{1}}\right) g\left(x_{1}, Q^{2}\right)\right)\right] .
$$


onde $q_{S}\left(x, Q^{2}\right)$ é a distribuição de quarks singleto:

$$
q_{S}\left(x, Q^{2}\right)=\sum_{i}\left[q_{i}\left(x, Q^{2}\right)+\bar{q}_{i}\left(x, Q^{2}\right)\right]
$$

As funções $P_{i j}\left(P_{q q}, P_{q g}, P_{g q}, P_{g g}\right)$ são denominadas funções de splitting. Em ordem dominante de $\alpha_{s}$ estas funções são dadas por:

$$
\begin{aligned}
& P_{q q}^{(0)}(z)=C_{F}\left[\frac{1+z^{2}}{(1-z)_{+}}+\frac{3}{2} \delta(1-z)\right] \\
& P_{q g}^{(0)}(z)=\frac{1}{2}\left[z^{2}+(1-z)^{2}\right] \\
& P_{g q}^{(0)}(z)=C_{F}\left[\frac{1+(1-z)^{2}}{z}\right] \\
& P_{g g}^{(0)}(z)=2 C_{A}\left[\frac{z}{(1-z)_{+}}+\frac{1-z}{z}+z(1-z)\right]+\frac{11 C_{A}-2 n_{f}}{6} \delta(1-z)
\end{aligned}
$$

onde $C_{F}=\left(N_{C}^{2}-1\right) / 2 N_{C}, C_{A}=N_{C}$ e $n_{f}$ é o número de sabores; aqui identificamos $z=x / x_{1}$. As distribuições "+" são dadas por:

$$
\int_{0}^{1} d x \frac{f(x)}{(1-x)_{+}}=\int_{0}^{1} d x \frac{f(x)-f(1)}{1-x} .
$$

As funções de splitting $P_{i j}$ podem ser interpretadas como a probabilidade de um parton $i$ emitir um parton $j$.

A QCD perturbativa permite determinar somente a evolução das distribuições partônicas (através das equações DGLAP). As condições iniciais para a evolução são de natureza não-perturbativa, devendo ser extraídas do experimento para uma dada virtualidade do fóton. Como as distribuições partônicas são universais, independentes do processo considerado, após determiná-las em um certo valor de $Q^{2}$ podemos, com o auxílio das equações DGLAP, determinar seus valores para outra virtualidade e assim utilizá-las no cálculo do processo de interesse.

A violação do scaling de Bjorken, onde $F_{2}$ se torna uma função de duas variáveis $\left(x\right.$ e $\left.Q^{2}\right)$, foi verificada experimentalmente. Esse comportamento é muito bem descrito 


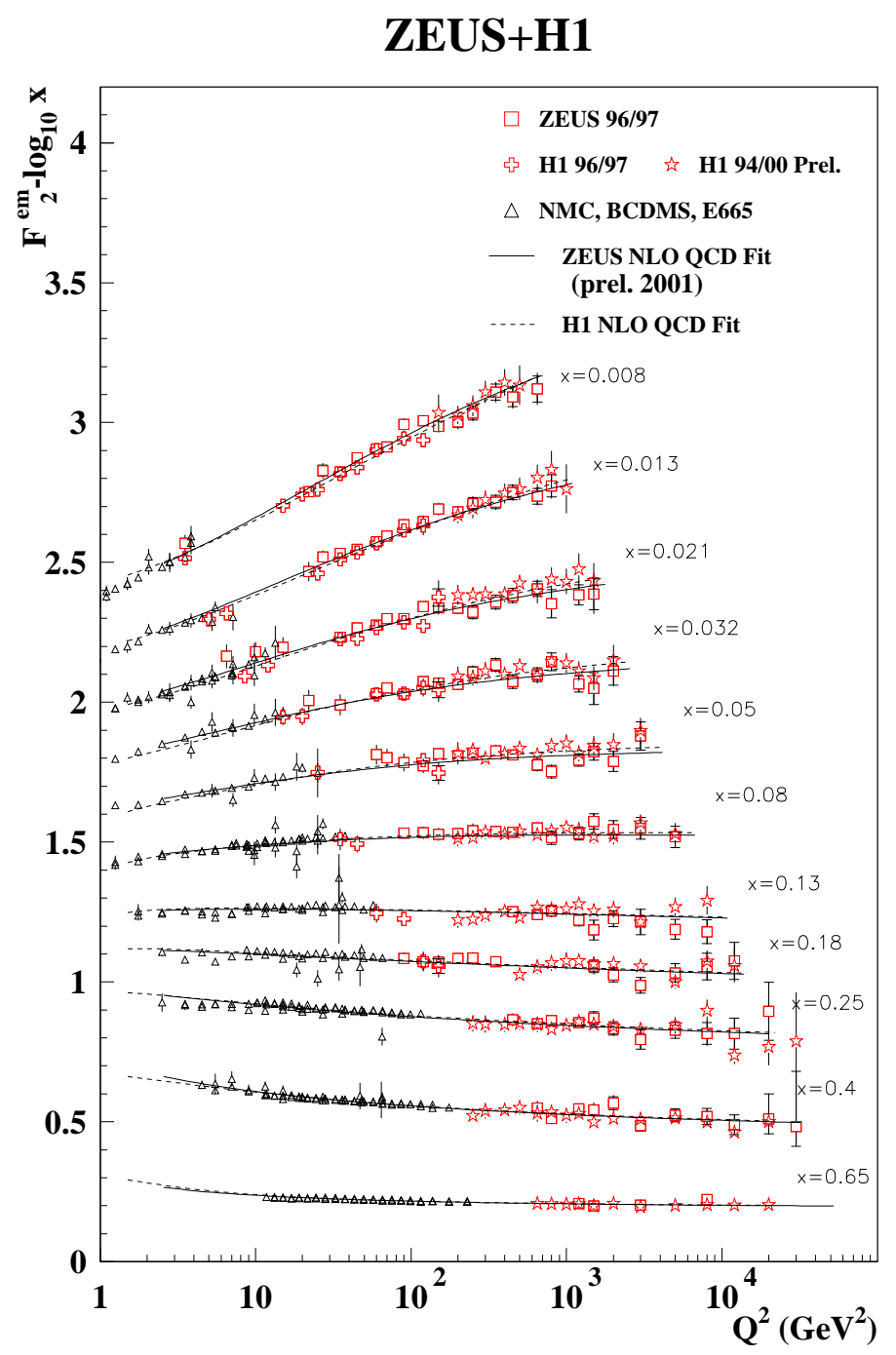

Figura 1.3: Função de estrutura $F_{2}$ (Extraído de [11]).

pela equação de DGLAP mesmo em ordem dominante (da expansão na constante de acoplamento), como pode ser visto nas figuras 1.3 e 1.4 . Para $x>0,5 \quad F_{2}$ diminui com o aumento de $Q^{2}$, enquanto que para $x<0,1 \quad F_{2}$ aumenta com $Q^{2}$.

Atualmente, existem na literatura diversos grupos que se dedicam a obter parametrizações para as distribuições partônicas, i.e., soluções das equações de DGLAP para todos os tipos de partons. Nesta dissertação utilizaremos as parametrizações de GRV-98 [12] para as distribuições partônicas do nucleon. Em [12] os parâmetros de entrada foram escolhidos apropriadamente para descrever os dados experimentais de $F_{2}^{p}\left(x, Q^{2}\right)$ 


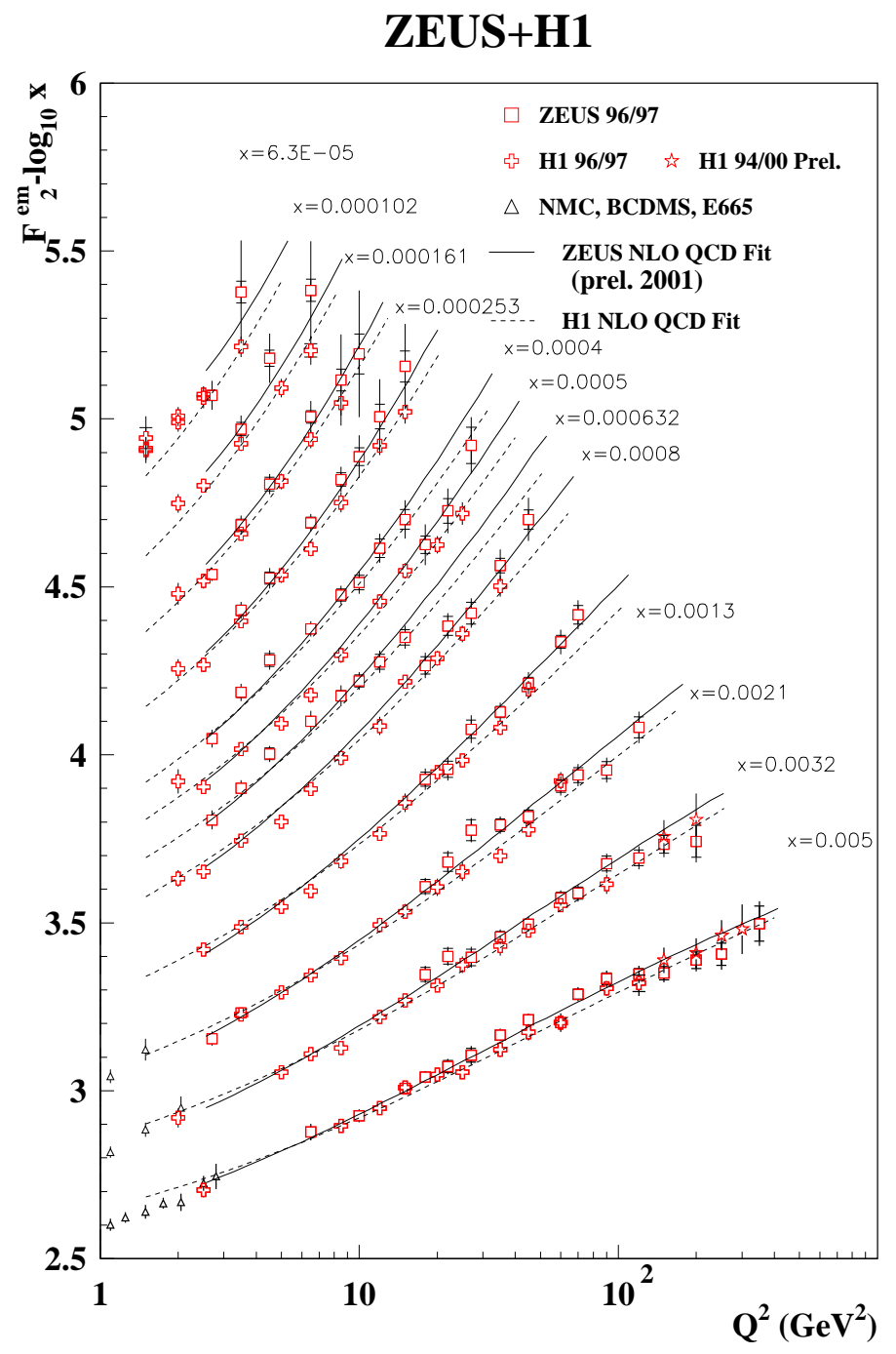

Figura 1.4: Função de estrutura $F_{2}$ (Extraído de [11]).

(função de estrutura do próton) mais recentes da época (1998) obtidos em HERA. Nós utilizamos estas distribuições partônicas em nossos cálculos através de um pacote, em linguagem FORTRAN, que os autores disponibilizaram [12].

Conforme veremos no capítulo 3, existe uma outra equação de evolução, chamada Equação de BFKL, em que o momento transferido $Q^{2}$ é fixado e a distribuição de glúons é evoluída em energia, ou seja, é evoluída em termos de $\ln (1 / x)$. 


\subsection{A equação de DGLAP no limite de pequeno $x$}

Consideremos as equações DGLAP apresentadas na seção anterior. A partir da análise das funções de splitting $P_{i j}$ verifica-se que as funções associadas ao setor de glúons $P_{g q}(z)$ e $P_{g g}(z)$ são singulares para $z \rightarrow 0$, e/ou $x \rightarrow 0$, pois como definido anteriormente $z=\frac{x}{x_{1}}$. As demais funções de splitting, associadas ao setor de quarks, são regulares neste limite. Consequentemente, o comportamento das distribuições partônicas em pequeno $x$ é determinado predominantemente pela dinâmica gluônica.

Em vista disso, podemos aproximar a equação de DGLAP para $g\left(x, Q^{2}\right)$ na região de pequeno $x$ por [3]:

$$
\frac{\partial g\left(x, Q^{2}\right)}{\partial \ln Q^{2}}=\frac{\alpha_{s}}{2 \pi} \int_{x}^{1} \frac{d x_{1}}{x_{1}} \mathcal{P}_{g g}\left(\frac{x}{x_{1}}\right) g\left(x_{1}, Q^{2}\right)
$$

onde $\mathcal{P}_{g g}(z)=P_{g g}^{z \rightarrow 0}(z)=\frac{2 C_{A}}{z}$ representa o termo singular na função de splitting. Substituindo $\mathcal{P}_{g g}(z)$ na equação $(1,4.33)$ temos

$$
\frac{\partial g\left(x, Q^{2}\right)}{\partial \ln Q^{2}}=\frac{\alpha_{s}}{2 \pi} \int_{x}^{1} \frac{d x_{1}}{x_{1}} \frac{2 C_{A}}{z} g\left(x_{1}, Q^{2}\right)
$$

ou ainda:

$$
\begin{aligned}
g\left(x, Q^{2}\right) & =\frac{\alpha_{s} C_{A}}{\pi} \int_{x}^{1} \frac{d x_{1}}{x_{1}} \frac{1}{z} \int_{Q_{0}^{2}}^{Q^{2}} \frac{d k_{T}^{2}}{k_{T}^{2}} g\left(x_{1}, k_{T}^{2}\right) \\
& =\frac{\alpha_{s} C_{A}}{\pi} \int_{x}^{1} \frac{d x_{1}}{x_{1}} \frac{x_{1}}{x} \int \frac{d k_{T}^{2}}{k_{T}^{2}} g\left(x_{1}, k_{T}^{2}\right)
\end{aligned}
$$

Portanto,

$$
x g\left(x, Q^{2}\right)=\int_{x}^{1} \frac{d x_{1}}{x_{1}} \int_{Q_{0}^{2}}^{Q^{2}} \frac{d k_{T}^{2}}{k_{T}^{2}} \frac{\alpha_{s} N_{c}}{\pi} x_{1} g\left(x_{1}, k_{T}^{2}\right)
$$

onde introduzimos um corte $Q_{0}$ na integração sobre os momentos transversais para separar a contribuição não perturbativa, e usamos que $C_{A}=N_{c}$. Definindo $y=$ 
$\ln (1 / x)$ e $\Gamma=\ln \left(Q^{2} / Q_{0}^{2}\right)$ temos:

$$
x g\left(x, Q^{2}\right)=\int_{y} d y^{\prime} \int_{\Gamma} d \Gamma^{\prime} \frac{\alpha_{s} N_{c}}{\pi} x^{\prime} g\left(x^{\prime}, k^{2}\right) .
$$

A solução da equação $(1,4.37)$ pode ser obtida facilmente quando consideramos que a constante de acoplamento está fixa. Introduzimos a transformada de Mellin de $x g\left(x, Q^{2}\right) \equiv G(y, \Gamma)$, definida por

$$
G(\omega, \Gamma)=\int d y e^{\omega y} G(y, \Gamma)
$$

e sua inversa por

$$
G(y, \Gamma)=\frac{1}{2 \pi i} \int_{-i \infty}^{+i \infty} d \omega e^{\omega y} G(\omega, \Gamma)
$$

onde o contorno de integração localiza-se à direita de todas as singularidades de $G(\omega, \Gamma)$, sendo $\omega$ a variável conjugada a $y[4]$. Assim,

$$
\begin{aligned}
G(\omega, \Gamma) & =\int d y e^{\omega y} \int_{y} d y^{\prime} \int_{\Gamma} d \Gamma^{\prime} \frac{\alpha_{s} N_{c}}{\pi} G\left(y^{\prime}, \Gamma^{\prime}\right) \\
& =\frac{\alpha_{s} N_{c}}{\pi} \int d y^{\prime} \int_{\Gamma} d \Gamma^{\prime} \int d y e^{\omega y} \Theta\left(y^{\prime}-y\right) G\left(y^{\prime}, \Gamma^{\prime}\right) \\
& =\frac{\alpha_{s} N_{c}}{\omega \pi} \int d y^{\prime} \int_{\Gamma} d \Gamma^{\prime} e^{\omega y^{\prime}} G\left(y^{\prime}, \Gamma^{\prime}\right) \\
& =\frac{\alpha_{s} N_{c}}{\omega \pi} \int_{\Gamma} d \Gamma^{\prime} \int d y^{\prime} e^{\omega y^{\prime}} G\left(y^{\prime}, \Gamma^{\prime}\right) \\
& =\frac{\alpha_{s} N_{c}}{\omega \pi} \int_{\Gamma} d \Gamma^{\prime} G\left(\omega, \Gamma^{\prime}\right) .
\end{aligned}
$$

Derivando a expressão acima com respeito a $\Gamma^{\prime}$, obtemos:

$$
\begin{aligned}
\frac{d G}{d \Gamma^{\prime}} & =\frac{\alpha_{s} N_{c}}{\omega \pi} G\left(\omega, \Gamma^{\prime}\right) \\
\frac{d G}{G} & =\frac{\alpha_{s} N_{c}}{\omega \pi} d \Gamma^{\prime} .
\end{aligned}
$$


A solução desta equação é

$$
G(\omega, \Gamma)=G\left(\omega, \Gamma_{0}\right) e^{\frac{\alpha_{s} N_{c}}{\pi \omega} \Gamma}
$$

O termo $\frac{\alpha_{s} N_{c}}{\pi \omega}$ é denominado dimensão anômala. O crescimento da distribuição de glúons na região de pequeno $x$ (ou pequeno $\omega$ ) está associado ao fato de que a dimensão anômala torna-se muito grande para $\omega \rightarrow 0$.

Agora, para obter a distribuição de glúons fazemos a transformada inversa de Mellin

$$
G(y, \Gamma)=\frac{1}{2 \pi i} \int d \omega e^{\omega y} G\left(\omega, \Gamma_{0}\right) e^{\frac{\alpha_{s} N_{c}}{\pi \omega} \Gamma}
$$

Na região de pequeno $x$ (grande $y$ ) e grande $\Gamma$ podemos utilizar o método de ponto de sela no cálculo da integral (1,4.43). Escrevendo por extenso o expoente de $(1,4.43)$ temos:

$$
u=\omega y+\frac{\alpha_{s} N_{c}}{\pi \omega} \Gamma
$$

O ponto de sela é determinado a partir da condição de mínimo, ou seja,

$$
\frac{\partial u}{\partial \omega}=0
$$

e assim o ponto de sela $\omega_{s}$ é:

$$
\omega_{s}=\sqrt{\frac{\alpha_{s} N_{c} \Gamma}{\pi y}} .
$$

Expandindo $u$ até segunda ordem em torno do ponto de sela $\omega_{s}$ :

$$
u=u\left(\omega_{s}\right)+\left.\frac{\partial u}{\partial \omega}\right|_{\omega_{s}}\left(\omega-\omega_{s}\right)+\left.\frac{1}{2} \frac{\partial^{2} u}{\partial \omega^{2}}\right|_{\omega_{s}}\left(\omega-\omega_{s}\right)^{2}+\ldots
$$

A segunda derivada calculada em $\omega_{s}$ é:

$$
\left.\frac{\partial^{2} u}{\partial \omega^{2}}\right|_{\omega_{s}}=\left.\frac{2 \alpha_{s} N_{C}}{\pi \omega^{3}} \Gamma\right|_{\omega_{s}}=\frac{2 \alpha_{s} N_{C}}{\pi \omega_{s}^{3}} \Gamma
$$


Substituindo a expansão acima em $(1,4.43)$, obtemos

$$
G(y, \Gamma)=\frac{1}{2 \pi i} \int_{-i \infty}^{+i \infty} d \omega G\left(\omega, \Gamma_{0}\right) \exp \left[u\left(\omega_{s}\right)+\frac{1}{2} u^{\prime \prime}\left(\omega-\omega_{s}\right)^{2}\right]
$$

fazendo a seguinte substituição: $\omega-\omega_{s}=i \nu \Rightarrow d \omega=i d \nu$,

$$
G(y, \Gamma)=\frac{1}{2 \pi} \int_{-\infty}^{+\infty} d \nu G\left(\omega_{0}, \Gamma_{0}\right) \exp \left[u\left(\omega_{s}\right)+\frac{1}{2} u^{\prime \prime}\left(-\nu^{2}\right)\right]
$$

onde identificamos uma integral gaussiana. Resolvendo-a obtemos

$$
\begin{aligned}
G(y, \Gamma) & =\frac{G\left(\omega_{0}, \Gamma_{0}\right)}{2 \pi} \sqrt{\frac{\pi}{\frac{1}{2} u^{\prime \prime}}} \exp \left(u\left(\omega_{s}\right)\right) \\
& =\frac{G\left(\omega_{0}, \Gamma_{0}\right)}{2 \pi} \sqrt{\frac{\pi^{2} \omega_{s}^{3}}{\alpha_{s} N_{C} \Gamma}} \exp \left[\omega_{s} y+\frac{\alpha_{s} N_{c}}{\pi \omega_{s}} \Gamma\right]
\end{aligned}
$$

Substituindo o valor de $\omega_{s}$ obtemos finalmente

$$
G(y, \Gamma)=\frac{G\left(\omega_{0}, \Gamma_{0}\right)}{2}\left(\frac{\alpha_{s} N_{c} \Gamma}{\pi^{3} y^{3}}\right)^{\frac{1}{4}} e^{2 \sqrt{\frac{\alpha_{s} N_{c}}{\pi} \Gamma y}}
$$

Esta é a solução da equação de DGLAP para a distribuição de glúons na aproximação de duplo logarítmo dominante [3], DLLA, que é justamente a aproximação na qual retemos apenas os termos singulares nas funções de splitting em LO no limite $x \rightarrow 0$. Toda a dependência na condição inicial está concentrada na função $G\left(\omega_{0}, \Gamma_{0}\right)$. Observamos que a equação DGLAP prevê um forte crescimento da distribuição de glúons na região de pequeno $x$.

\subsection{O DIS Nuclear}

Na primeira seção deste capítulo nós discutimos a cinemática do espalhamento inelástico profundo, DIS, com um nucleon $N$ no estado inicial. Este processo é representado por $(1,1.1)$. Nos 2 trabalhos que desenvolvemos nós estudamos os processos de espal- 
hamento $e A(e+A \rightarrow e+X)$, onde $A$ representa um núcleo com número de massa $A$. Conforme veremos, os processos $e A$ poderão ser usados no estudo de duas questões muito importantes atualmente, a saturação de partons e a distribuição nuclear de glúons.

Os dados experimentais existentes atualmente sobre colisões $e A$ são bastante escassos e de pouca precisão. Há alguns anos vêm sendo discutidos projetos de construção de um Colisor Eletron-Íon (eletron-ion collider - EIC) [13], o qual nos fornecerá uma quantidade grande de dados de alta precisão deste tipo de processo. O design original do EIC envolve duas propostas [13]: a construção do eRHIC no lugar do atual RHIC, onde as energias envolvidas serão maiores do que as atuais, e a construção do ELIC no atual Jefferson Lab. O eRHIC permitirá experimentos com energias do centro de massa $\sqrt{s}=60-90 \mathrm{GeV}$ e menor luminosidade $\mathcal{L} \approx 10^{33} \mathrm{~cm}^{-2} \mathrm{~s}^{-1}$, enquanto o ELIC terá energias $\sqrt{s} \leq 60 \mathrm{GeV}$ e maior luminosidade $\mathcal{L} \approx 10^{35} \mathrm{~cm}^{-2} \mathrm{~s}^{-1}$.

Como nossos trabalhos envolvem processos $e A$ com pequenos valores de $x$ (maiores energias de centro de massa), o projeto eRHIC é o mais interessante para nós.

\subsection{Efeito de "Shadowing" Nuclear}

Os dados obtidos em experimentos de espalhamento inelástico profundo (Deep Inelastic Scattering - DIS) [14], analisados em uma grande região cinemática

$$
10^{-5} \leq x \leq 0,1 \quad, \quad 0,05 \mathrm{GeV}^{2} \leq Q^{2} \leq 100 \mathrm{GeV}^{2}
$$

mostram uma redução sistemática da função de estrutura nuclear $F_{2}^{A}\left(x, Q^{2}\right) / A$ com relação à função de estrutura do nucleon livre $F_{2}^{N}\left(x, Q^{2}\right)$, onde $A$ é o número de massa do núcleo.

Em outras palavras, a função de estrutura medida no nucleon ligado ao núcleo é menor do que a função de estrutura deste mesmo nucleon livre no espaço (veja figura 1.5). Este efeito é conhecido como "Nuclear Shadowing Effect", ou efeito de shadowing 


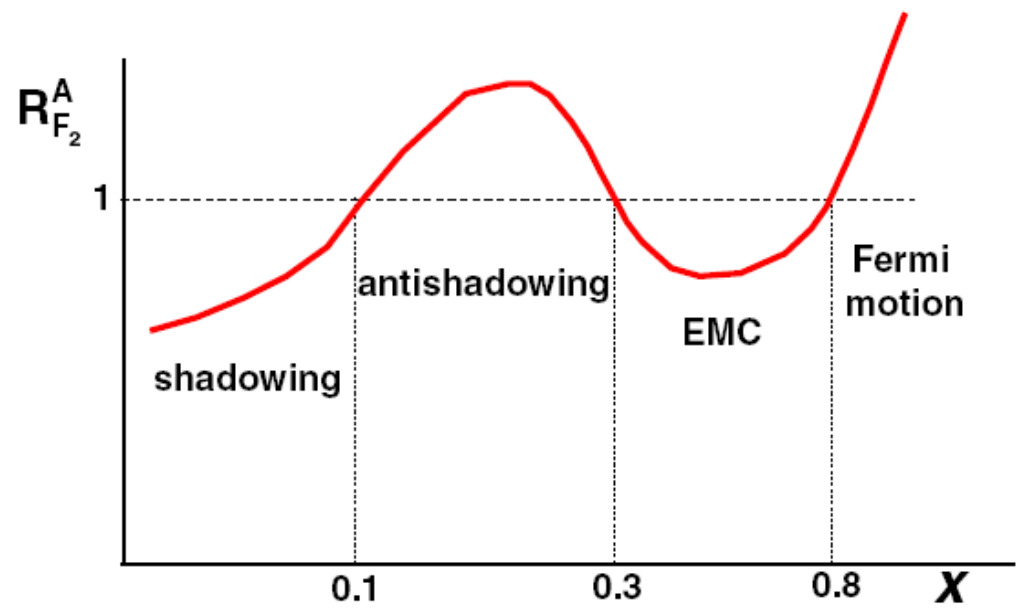

Figura 1.5: Razão $R_{F_{2}}^{A}=F_{2}^{A} /\left(A F_{2}^{N}\right)$ (Extraído de [15]) .

nuclear. A modificação na função de estrutura depende da fração $x$ de momento dos partons. O efeito de shadowing acontece para

$$
x<0,1 \quad, \quad 0,3<x<0,7
$$

Enquanto que um efeito contrário (antishadowing) acontece na região

$$
0,1<x<0,3
$$

Ou seja, nesta região há um efeito de aumento na função de estrutura do nucleon ligado ao núcleo com relação ao nucleon livre, contrário ao efeito de shadowing.

\subsection{Parametrizações das distribuições nucleares de partons}

Dada a distribuição $f_{i}\left(x, Q^{2}\right)$ de um determinado parton $f_{i}$ em um nucleon, para obter a distribuição $f_{i}^{A}$ deste parton em um núcleo de número de massa $A$ devemos 
multiplicar $f_{i}$ por um fator $R_{i}^{A}$, i.e.

$$
\frac{f_{i}^{A}\left(x, Q^{2}\right)}{A} \equiv R_{i}^{A}\left(x, Q^{2}\right) f_{i}\left(x, Q^{2}\right)
$$

O fator $R_{i}^{A}$ seria igual a 1 se as funções de estrutura dos partons de um núcleo fossem uma mera superposição das funções de estrutura dos partons de um nucleon. Porém, como discutido na seção anterior, existem os fenômenos de shadowing e antishadowing, dependendo do valor de $x$, que implicam que $R_{i}^{A}$ é menor ou maior que 1 respectivamente.

Os dados das funções de estrutura do próton são muito bem descritos, por exemplo pelas parametrizações de GRV para as distribuições partônicas. As distribuições de partons dos núcleos, em particular a distribuição de glúons (que se torna a mais importante em altas energias), ainda são bastante incertas.

Para podermos fazer um estudo quantitativo detalhado da distribuição nuclear de glúons nós utilizamos quatro diferentes parametrizações para os fatores $R_{i}^{A}$ partônicos, propostas por Eskola, Kolhinen e Salgado [16] (1999), de Florian e Sassot [17] (2004), Hirai, S. Kumano e T.H. Nagai [18] (2007) e K.J. Eskola, H.Paukkunen and C.A. Salgado [19] (2008). Vamos chamá-las respectivamente de EKS, DS, HKN e EPS.

Diferentes parametrizações diferem [20], por exemplo, na forma das parametrizações na escala inicial, no uso de diferentes conjuntos de dados experimentais, na ordem da evolução DGLAP, no tratamento de efeitos de isospin, entre outras coisas. Os grupos DS e HKN fornecem parametrizações em leading order (LO) e next-to-leading order (NLO), enquanto EKS e EPS fazem uma análise global apenas em LO. Para compará-los nós usamos LO para DS e HKN.

Os dados experimentais para a função de estrutura nuclear determinam o comportamento das distribuições nucleares dos quarks. O comportamento da distribuição nuclear de glúons é determinado indiretamente usando regra de soma de momentum e/ou estudando a inclinação em $\log Q^{2}$ da razão $F_{2}^{S n} / F_{2}^{C}[21]$. 


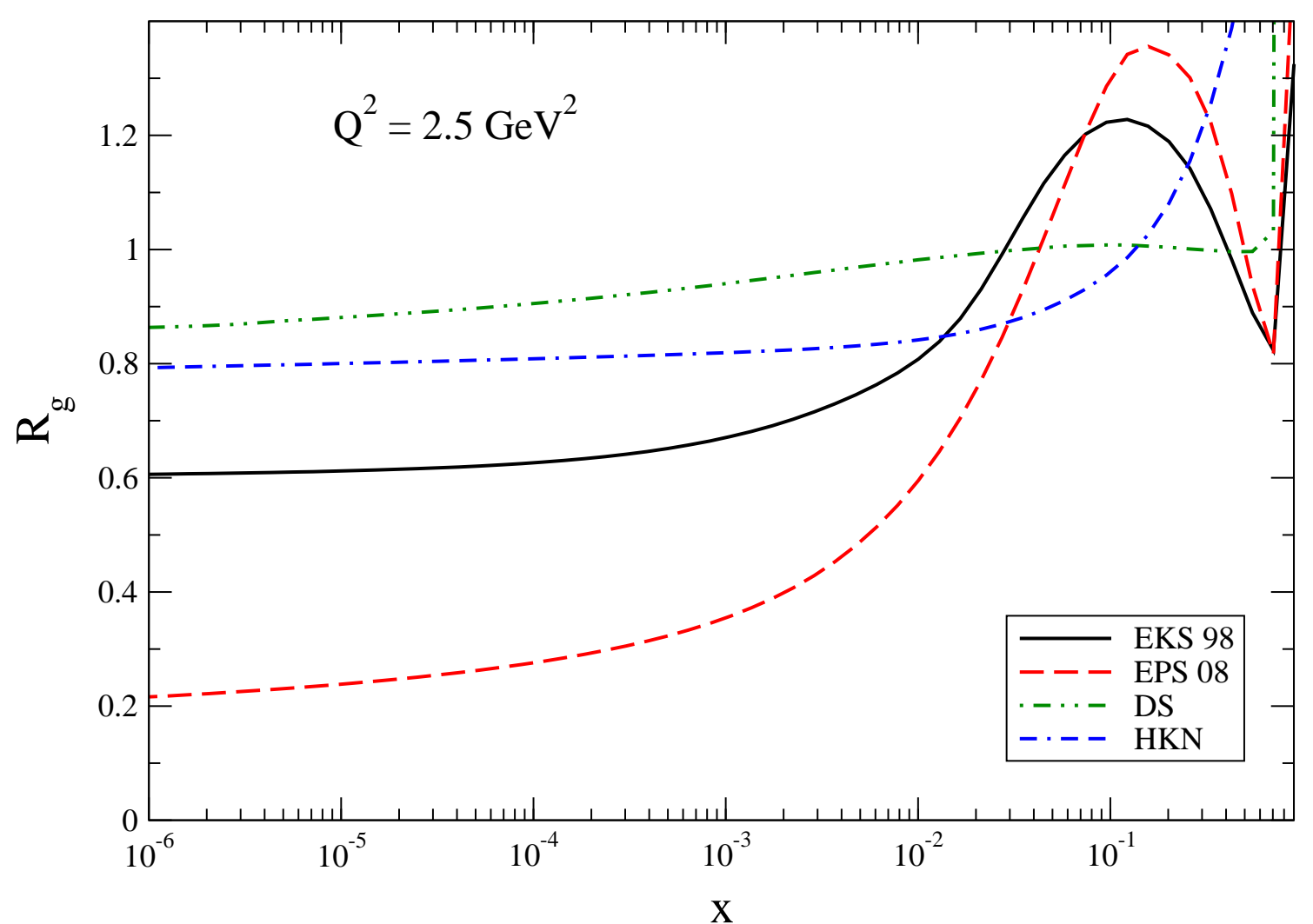

Figura 1.6: Razão $R_{g}=x g_{A} /\left(A x g_{N}\right)$ predita pelas parametrizações EKS, DS, HKN e EPS para $A=208$ e $Q^{2}=2,5 \mathrm{GeV}^{2}$.

Na figura 1.6 nós plotamos a razão

$$
R_{g}=\frac{x g_{A}}{A x g_{N}}
$$

para o chumbo, $A=208$, predita pelas quatro parametrizações, onde $x g_{A}\left(x g_{N}\right)$ é a função de distribuição de glúons no núcleo (nucleon).

Como se vê, o comportamento de $x g_{A}\left(x, Q^{2}\right)$ em pequeno $x$ é bastante incerto. Em particular, a magnitude do efeito de shadowing e a presença ou não do efeito de antishadowing é indefinida. 


\subsection{Funções de estrutura longitudinal e de charme}

A função de estrutura longitudinal $F_{L}$, em leading order (LO) na QCD, é dada pela equação de Altarelli-Martinelli [22, 23]

$$
F_{L}\left(x, Q^{2}\right)=\frac{\alpha_{s}\left(Q^{2}\right)}{2 \pi} x^{2} \int_{x}^{1} \frac{d y}{y^{3}}\left[\frac{8}{3} F_{2}\left(y, Q^{2}\right)+4 \sum_{q} e_{q}^{2}\left(1-\frac{x}{y}\right) y g\left(y, Q^{2}\right)\right]
$$

onde a soma é feita sobre três sabores.

Em pequeno $x$ o segundo termo (com a distribuição de glúons) é o termo dominante. Na Ref. [24] os autores sugeriram que, para pequeno $x$, esta expressão pode ser razoavelmente aproximada por

$$
F_{L}\left(x, Q^{2}\right) \approx 0,3 \frac{4 \alpha_{s}}{3 \pi} x g\left(2,5 x, Q^{2}\right)
$$

o que demonstra a íntima relação entre a função de estrutura longitudinal e a distribuição de glúons. Quando o alvo é um núcleo $g \rightarrow g_{A}$ e $F_{L} \rightarrow F_{L}^{A}$ e podemos definir a razão (ou fator de modificação)

$$
R_{L}=\frac{F_{L}^{A}}{A F_{L}^{N}}
$$

Um par $c \bar{c}$ pode ser criado pela fusão bóson-glúon [25] quando a massa invariante ao quadrado do estado final hadrônico é

$$
W^{2} \geq 4 m_{c}^{2}
$$

Como $W^{2}=Q^{2}(1-x) / x+M_{N}^{2}$, onde $M_{N}$ é a massa do nucleon, a produção de charm pode ocorrer bem abaixo do limiar $Q^{2} \approx 4 m_{c}^{2}$, para $x$ pequeno.

A contribuição $F_{2}^{c}$ do charm para a função de estrutura $F_{2}$ do próton (ou núcleo), 
em leading order (LO), é dada por [26]

$$
\frac{1}{x} F_{2}^{c}\left(x, Q^{2}, m_{c}^{2}\right)=2 e_{c}^{2} \frac{\alpha_{s}\left(\mu^{\prime 2}\right)}{2 \pi} \int_{a x}^{1} \frac{d y}{y} C_{g, 2}^{c}\left(\frac{x}{y}, \frac{m_{c}^{2}}{Q^{2}}\right) g\left(y, \mu^{\prime 2}\right)
$$

onde $a=1+4 m_{c}^{2} / Q^{2}$ e a escala de fatorização $\mu^{\prime}$ é assumida $\mu^{\prime 2}=4 m_{c}^{2}$. $C_{g, 2}^{c}$ é a função coeficiente dada por

$$
\begin{array}{r}
C_{g, 2}^{c}\left(z, \frac{m_{c}^{2}}{Q^{2}}\right)=\frac{1}{2} \ln \frac{1+\beta}{1-\beta}\left[z^{2}+(1-z)^{2}+z(1-3 z) \frac{4 m_{c}^{2}}{Q^{2}}-z^{2} \frac{8 m_{c}^{4}}{Q^{4}}\right]+ \\
\frac{\beta}{2}\left[-1+8 z(1-z)-z(1-z) \frac{4 m_{c}^{2}}{Q^{2}}\right]
\end{array}
$$

onde $\beta=1-4 m_{c}^{2} z / Q^{2}(1-z)$ é a velocidade de um dos quarks charm no referencial do centro de massa bóson-glúon.

Portanto, em LO, $\mathcal{O}\left(\alpha_{s}\right), F_{2}^{c}$ é diretamente sensível apenas à densidade de glúons, via o processo $\gamma^{*} g \rightarrow c \bar{c}$.

Assim como já foi feito antes, quando o alvo é um núcleo podemos definir a razão

$$
R_{C}=\frac{F_{2}^{c, A}}{A F_{2}^{c, N}}
$$

\subsection{Espalhamento difrativo}

Nos experimentos de espalhamento inelástico profundo (DIS) lépton-hádron e hádronhádron é observado, um certo número de vezes, o chamado Espalhamento Difrativo. Este tipo de processo é caracterizado pela existência de uma região do espaço de fase (no estado final da colisão) na qual não é observada nenhuma partícula. Esta região é chamada de "gap" de rapidez. O termo "difrativo" é justificado pelo fato de que o alvo (hádron) permanece intacto após a colisão, e assim podemos afirmar que nenhum número quântico é trocado entre o fóton virtual e o hádron no processo de espalhamento.

Na teoria de Regge um processo é difrativo quando, entre o projétil e o alvo, ocorre 
a troca de um objeto com os números quânticos do vácuo. Este objeto é chamado de Pomeron. Desde o início dos anos oitenta surgiram várias tentativas de entender o Pomeron no contexto da QCD. Uma destas tentativas foi apresentada por Low [27] e Nussinov [28]. Nela o Pomeron é formado por dois glúons num estado singleto de cor. Este modelo de troca de dois glúons continua a ser utilizado.

Uma fração significativa (em torno de 10\%) dos eventos de espalhamento inelástico profundo observados pelas colaborações ZEUS e H1 no HERA, são de natureza difrativa. Nestes eventos o próton que entra permanece intacto apesar da inelasticidade da reação, perdendo apenas uma pequena fração $x_{\mathbb{P}}$ de seu momento inicial. $\mathrm{O}$ estado final do próton está bem separado em rapidez do resto do sistema, o qual parece resultado de um evento de DIS típico. Em linguagem partônica, um aglomerado de partons neutro de cor fragmenta-se independentemente do próton espalhado. 


\section{Capítulo 2}

\section{Dipolos de cor}

\subsection{DIS na representação de dipolos de cor}

O formalismo de dipolo de cor é útil para o estudo do DIS no regime de $x$ pequeno, especialmente no referencial de repouso do alvo hadrônico. Neste referencial, quando $x \rightarrow 0$ o fóton virtual transforma-se em um par quark-antiquark e, depois de um tempo longo, ele colide com o alvo. Desde que o tempo de interação seja muito mais curto do que o tempo de vida do par, o tamanho transversal do par $q \bar{q}$ pode ser considerado fixo durante o processo de espalhamento [3, 5].

O momento do fóton virtual é:

$$
q=\left(\nu, 0,0, \sqrt{\nu^{2}+Q^{2}}\right)
$$

onde $Q^{2}=-q^{2}, \nu=\left(k-k^{\prime}\right)$ e $k$ e $k^{\prime}$ são os momentos do quark e do antiquark, respectivamente. Usando as variáveis do cone de luz escrevemos os momentos do fóton virtual $\left(\gamma^{*}\right)$, do quark $(q)$ e do anti-quark $(\bar{q})$ como:

$$
\begin{aligned}
& \gamma^{*}: \quad=\left(q^{+},-\frac{Q^{2}}{2 q^{+}}, \overrightarrow{0}\right) ; \\
& q: \quad k=\left(z q^{+}, \frac{\vec{k}^{2}}{2 z q^{+}}, \vec{k}\right) ;
\end{aligned}
$$




$$
\bar{q}: \quad k^{\prime}=\left((1-z) q^{+}, \frac{\vec{k}^{2}}{2(1-z) q^{+}},-\vec{k}\right),
$$

onde $z$ é a fração de momento do fóton carregada pelo quark ((1-z) pelo anti-quark) e $q^{+} \simeq \sqrt{2} \nu$ no limite de Bjorken. A massa invariante quadrada do par $q \bar{q}$ é:

$$
M^{2}=\left(k+k^{\prime}\right)^{2}=\frac{\vec{k}^{2}}{z(1-z)} .
$$

Usando o princípio da incerteza podemos estimar o tempo de vida $\tau_{f}$ do par:

$$
\tau_{f} \sim \frac{1}{\Delta E}
$$

onde $\Delta E=E_{\text {par }}-E_{\gamma^{*}}$, sendo

$$
E_{p a r}=\frac{1}{\sqrt{2}}\left(q^{+}+\frac{\vec{k}^{2}}{2 z(1-z) q^{+}}\right)
$$

e

$$
E_{\gamma^{*}}=\frac{1}{\sqrt{2}}\left(q^{+}-\frac{Q^{2}}{2 q^{+}}\right)
$$

Assim

$$
\Delta E=\frac{1}{2 \sqrt{2} q^{+}}\left(Q^{2}+\frac{k^{2}}{z(1-z)}\right),
$$

e considerando que $Q^{2} \gg M^{2}$ temos:

$$
\Delta E \simeq \frac{Q^{2}}{\sqrt{2} q^{+}}=m_{N} x
$$

Finalmente concluímos que:

$$
\tau_{f} \sim \frac{1}{\Delta E} \sim \frac{1}{m_{N} x}
$$

Vemos então que quando $x \rightarrow 0 \quad \tau_{f}$ é muito maior que o tempo típico de interação $\tau_{\text {int }}$, pois $\tau_{\text {int }} \sim R_{A}$, onde $R_{A}$ é o raio do alvo. Consequentemente, em $x$ pequeno podemos interpretar o DIS como o espalhamento de um dipolo de cor $q \bar{q}$ com o hádron e considerar o tamanho transverso do par fixo durante a interação. Isto nos leva a 
pensar que a descrição da seção de choque $\gamma^{*} p$ pode ser fatorizada da seguinte maneira [3]

$$
\sigma^{\gamma^{*} p}=\int d z \int d r^{2}|\Psi|^{2} \sigma_{d i p}
$$

onde $r$ é o raio transversal do par $q \bar{q}, \Psi$ é a sua função de onda, que descreve a transição fóton virtual-dipolo, e $\sigma_{d i p}$ é a seção de choque de interação entre o par e o alvo. Nikolaev e Zakharov e posteriormente Mueller [29] demonstraram em ordem dominante ("leading order", LO) que esta expressão de fato pode ser obtida no formalismo da fatorização $k_{T}$, o que será discutido na próxima seção.

\subsubsection{Fatorização $k_{T}$ e a seção de choque de dipolo}

Em altas energias $(s \rightarrow \infty$, ou seja, $x \rightarrow 0$ ) a fatorização colinear (que dá origem às equações de DGLAP) deve ser generalizada de forma a permitir que o parton incidente possua momento transversal não-nulo [3]. É possível deduzir uma nova e mais geral fórmula de fatorização, chamada de "fatorização $k_{T}$ ". Com esta nova fórmula podemos escrever a contribuição do processo $\gamma^{*} g \rightarrow q \bar{q}$ para a seção de choque total $\gamma^{*} p$ da seguinte forma [5]:

$$
\sigma_{\lambda}^{\gamma^{*} p}\left(x, Q^{2}\right)=\int \frac{d \vec{K}^{2}}{\vec{K}^{2}} \int_{x}^{1} \frac{d x^{\prime}}{x^{\prime}} f\left(\frac{x}{x^{\prime}}, \vec{K}^{2}\right) \hat{\sigma}_{\lambda}^{\gamma^{*} g}\left(x^{\prime}, \vec{K}^{2}, Q^{2}\right)
$$

onde $\lambda$ denota a polarização do fóton virtual, $\hat{\sigma}_{\lambda}^{\gamma^{*} g}$ é a seção de choque do processo elementar $\gamma^{*} g \rightarrow q \bar{q}$ e $f\left(\frac{x}{x^{\prime}}, \vec{K}^{2}\right)$ é a distribuição de glúons não integrada, que está relacionada com a distribuição usual de glúons da seguinte forma:

$$
x g\left(x, Q^{2}\right)=\int^{Q^{2}} \frac{d \vec{K}^{2}}{\vec{K}^{2}} f\left(x, \vec{K}^{2}\right)
$$

A seção de choque do processo elementar

$$
\gamma^{*}(q)+g(K) \rightarrow q\left(k_{1}\right)+\bar{q}\left(k_{2}\right)
$$


é dada por [5]:

$$
\sigma_{\lambda}^{\gamma^{*} g}=\frac{\beta}{8 \pi^{2} W^{4} \vec{K}^{2}} \int \frac{d z}{z(1-z)} \int d^{2} \vec{k} \cdot \delta\left(\beta-\frac{z \vec{k}^{2}+(1-z)(\vec{k}+\vec{K})^{2}+\varepsilon^{2}}{z(1-z) W^{2}}\right) H_{\lambda}
$$

onde

$$
\varepsilon^{2} \equiv Q^{2} z(1-z)
$$

$\beta=x / x^{\prime}$ é a fração do momento longitudinal do próton carregada pelo glúon, $W$ é a energia de centro de massa do sistema fóton virtual - próton, e

$$
\begin{aligned}
H_{\lambda}= & 8 \pi^{2} \alpha_{s} \alpha_{e m} e_{q}^{2} \frac{z(1-z)}{Q^{2}} W^{4} \\
& \cdot\left\{\frac{N_{\lambda}(\vec{k}, \vec{k})}{\left(\vec{k}^{2}+\varepsilon^{2}\right)^{2}}+\frac{N_{\lambda}(\vec{k}+\vec{K}, \vec{k}+\vec{K})}{\left.\left[(\vec{k}+\vec{K})^{2}+\varepsilon^{2}\right)\right]^{2}}-\frac{2 N_{\lambda}(\vec{k}, \vec{k}+\vec{K})}{\left.\left(\vec{k}^{2}+\varepsilon^{2}\right)\left[(\vec{k}+\vec{K})^{2}+\varepsilon^{2}\right)\right]}\right\}
\end{aligned}
$$

com

$$
\begin{aligned}
& N_{L}\left(\vec{K}_{1}, \vec{K}_{2}\right)=4 z^{2}(1-z)^{2} Q^{4}, \\
& N_{T}\left(\vec{K}_{1}, \vec{K}_{2}\right)=Q^{2}\left[z^{2}+(1-z)^{2}\right] K_{1} \cdot K_{2} .
\end{aligned}
$$

Com a expressão acima a seção de choque $\gamma^{*} p$ pode ser reescrita como:

$$
\begin{aligned}
\sigma_{L, T}^{\gamma^{*} p}\left(x, Q^{2}\right) & =\frac{\alpha_{e m}}{Q^{2}} \sum_{q} e_{q}^{2} \int \frac{d \vec{K}^{2}}{\vec{K}^{4}} \int_{0}^{1} d z \int d^{2} \vec{k} \alpha_{s}\left(\mu^{2}\right) f\left(\beta, \vec{K}^{2}\right) \\
& \times\left\{\frac{N_{\lambda}(\vec{k}, \vec{k})}{\left(\vec{k}^{2}+\varepsilon^{2}\right)^{2}}+\frac{N_{\lambda}(\vec{k}+\vec{K}, \vec{k}+\vec{K})}{\left.\left[(\vec{k}+\vec{K})^{2}+\varepsilon^{2}\right)\right]^{2}}-\frac{2 N_{\lambda}(\vec{k}, \vec{k}+\vec{K})}{\left.\left(\vec{k}^{2}+\varepsilon^{2}\right)\left[(\vec{k}+\vec{K})^{2}+\varepsilon^{2}\right)\right]}\right\}
\end{aligned}
$$


Usando as seguintes identidades:

$$
\begin{aligned}
\int \frac{d^{2} \vec{k}}{\left(\vec{k}^{2}+\varepsilon^{2}\right)\left[(\vec{k}+\vec{K})^{2}+\varepsilon^{2}\right]}= & \frac{1}{(2 \pi)^{2}} \int d^{2} \overrightarrow{k_{1}} \int d^{2} \overrightarrow{k_{2}} \int d^{2} \vec{r} \\
& \times \frac{e^{i \vec{r} \cdot\left(\overrightarrow{k_{1}}+\vec{K}\right)-i \vec{r} \cdot \overrightarrow{k_{2}}}}{\left({\overrightarrow{k_{1}}}^{2}+\varepsilon^{2}\right)\left(\vec{k}_{2}^{2}+\varepsilon^{2}\right)} \\
= & \int d^{2} \vec{r} e^{i \vec{r} \cdot \vec{k}}\left|\frac{1}{2 \pi} \int d^{2} \vec{k} \frac{e^{i \vec{k} \cdot \vec{r}}}{\vec{k}^{2}+\varepsilon^{2}}\right|^{2}
\end{aligned}
$$

e

$$
\begin{aligned}
\int d^{2} \vec{k} \frac{\vec{k}^{2}+\vec{k} \cdot \vec{K}}{\left.\left(\vec{k}^{2}+\varepsilon^{2}\right)\left[(\vec{k}+\vec{K})^{2}+\varepsilon^{2}\right)\right]}= & \frac{1}{(2 \pi)^{2}} \int d^{2} \overrightarrow{k_{1}} \int d^{2} \overrightarrow{k_{2}} \int d^{2} \vec{r} \\
& \times \frac{\left(\vec{\nabla} e^{i \vec{r} \cdot\left(\overrightarrow{k_{1}}+\vec{K}\right)}\right) \cdot\left(\vec{\nabla} e^{-i \vec{r} \cdot \overrightarrow{k_{2}}}\right)}{\left(\vec{k}_{1}^{2}+\varepsilon^{2}\right)\left(\vec{k}_{2}^{2}+\varepsilon^{2}\right)}
\end{aligned}
$$

onde $\vec{\nabla} \equiv \partial / \partial \vec{r}$, é possível [5] reescrever $(2,1.7)$, com $\alpha_{s}$ fixo, na representação do parâmetro de impacto. Assim passamos do espaço dos momentos transversais para o espaço das coordenadas transversais, onde a variável $r$ será identificada com a separação transversal do dipolo $q \bar{q}$. Após integrações angulares (que fazem aparecer as funções de Bessel) e um reagrupamento de termos, chega-se a:

$$
\sigma_{L, T}^{\gamma^{*} p}\left(x, Q^{2}\right)=\int_{0}^{1} d z \int d^{2} \vec{r}\left|\Psi_{L, T}(z, r)\right|^{2} \sigma_{d i p}(x, r)
$$

onde

$$
\begin{aligned}
\left|\Psi_{L}(z, r)\right|^{2} & =\frac{6 \alpha_{e m}}{(2 \pi)^{2}} \sum_{q} 4 e_{q}^{2} Q^{2} z^{2}(1-z)^{2} K_{0}^{2}(\varepsilon r) \\
\left|\Psi_{T}(z, r)\right|^{2} & =\frac{6 \alpha_{e m}}{(2 \pi)^{2}} \sum_{q} e_{q}^{2}\left[z^{2}+(1-z)^{2}\right] \varepsilon^{2} K_{1}^{2}(\varepsilon r)
\end{aligned}
$$

$\mathrm{e}$

$$
\sigma_{d i p}(x, r)=\frac{4 \pi}{3} \int \frac{d^{2} \vec{K}}{\vec{K}^{4}} \alpha_{s} f\left(x, \vec{K}^{2}\right)\left(1-e^{i \vec{K} \cdot \vec{r}}\right)
$$


onde $L$ e $T$ significam respectivamente polarizações longitudinal e transversal do fóton.

A relação $(2,1.10)$ é independente de referencial. Podemos interpretar $\left|\Psi_{L, T}(z, r)\right|^{2}$ como sendo a probabilidade do fóton virtual flutuar em um par quark-antiquark. A interação do dipolo $q \bar{q}$ com o alvo está representada pela quantidade $\sigma_{\text {dip }}(x, r)$. Por sua vez $\sigma_{\text {dip }}(x, r)$ está relacionada com a distribuição de glúons não integrada.

\subsection{O espalhamento difrativo no formalismo de dipolo de cor}

A difração é essencialmente um efeito da mecânica quântica, e a expressão para a seção de choque difrativa pode ser derivada com base em seu formalismo [3, 5], como veremos à seguir.

Seja $T$ a matriz de transição que descreve os espalhamentos elástico e difrativo de um hádron $N$. Supondo que a amplitude de espalhamento seja puramente imaginária: $T=i \mathcal{D}$, onde $\mathcal{D}$ é real, vamos considerar uma base de estados hadrônicos físicos $|i\rangle$. O espalhamento difrativo leva um desses estados em outro e o espalhamento elástico leva cada estado nele mesmo. Assim $\mathcal{D}_{i k} \equiv\langle k|\mathcal{D}| i\rangle$ é a amplitude para a transição difrativa, $|k\rangle \rightarrow|i\rangle$. Os elementos da diagonal $\mathcal{D}_{i i} \equiv\langle i|\mathcal{D}| i\rangle$ são as amplitudes elásticas.

Introduzimos um conjunto completo de auto-estados de $\mathcal{D}$ :

$$
\mathcal{D}|\alpha\rangle=d_{\alpha}|\alpha\rangle
$$

O auto-valor $d_{\alpha}$ é proporcional à seção de choque total para o espalhamento $\alpha N$, que chamamos $\sigma_{\alpha}$ :

$$
\begin{aligned}
\sigma_{\alpha} & \equiv \sigma_{\text {tot }}^{\alpha N}=\frac{1}{s} \operatorname{Im}\langle\alpha|i \mathcal{D}| \alpha\rangle \\
& =\frac{1}{s} \operatorname{Im}\left(i\langle\alpha \mid \alpha\rangle d_{\alpha}\right)=\frac{1}{s} d_{\alpha} .
\end{aligned}
$$


Podemos expandir o estado físico $|i\rangle$ em termos de $|\alpha\rangle$ :

$$
|i\rangle=\sum_{\alpha} c_{i \alpha}|\alpha\rangle
$$

Os elementos de matriz do operador $\mathcal{D}$ são:

$$
\begin{aligned}
\mathcal{D}_{i k} & =\langle k|\mathcal{D}| i\rangle \\
& =\sum_{\alpha} \sum_{\beta} c_{k \beta}^{*} c_{i \alpha}\langle\beta|\mathcal{D}| \alpha\rangle \\
& =\sum_{\alpha} \sum_{\beta} c_{k \beta}^{*} c_{i \alpha}\langle\beta \mid \alpha\rangle d_{\alpha} \\
& =\sum_{\alpha} c_{k \alpha}^{*} c_{i \alpha} d_{\alpha} .
\end{aligned}
$$

A amplitude de espalhamento elástico fica:

$$
\begin{aligned}
\mathcal{D}_{i i} & =\langle i|\mathcal{D}| i\rangle \\
& =\sum_{\alpha} c_{i \alpha}^{*} c_{i \alpha} d_{\alpha} \\
& =\sum_{\alpha}\left|c_{i \alpha}\right|^{2} d_{\alpha}
\end{aligned}
$$

e a seção de choque total do espalhamento iN é obtida imediatamente, via teorema ótico:

$$
\begin{aligned}
\sigma_{\text {tot }}^{i N} & =\frac{1}{s} \mathcal{D}_{i i} \\
& =\sum_{\alpha}\left|c_{i \alpha}\right|^{2} \frac{d_{\alpha}}{s} \\
& =\sum_{\alpha}\left|c_{i \alpha}\right|^{2} \sigma_{\alpha} .
\end{aligned}
$$


O valor esperado de um operador $\mathcal{O}$ no estado $|i\rangle$ é:

$$
\begin{aligned}
\langle\mathcal{O}\rangle & \equiv\langle i|\mathcal{O}| i\rangle \\
& =\sum_{\alpha \beta}\langle i \mid \alpha\rangle\langle\alpha|\mathcal{O}| \beta\rangle\langle\beta \mid i\rangle \\
& =\sum_{\alpha \beta} c_{i \alpha} c_{i \beta}^{*}\langle\alpha|\mathcal{O}| \beta\rangle .
\end{aligned}
$$

Se $\mathcal{O}$ é diagonal na base $|\alpha\rangle$, então:

$$
\langle\mathcal{O}\rangle=\sum_{\alpha}\left|c_{i \alpha}\right|^{2} \mathcal{O}_{\alpha}
$$

onde $\mathcal{O}_{\alpha} \equiv\langle\alpha|\mathcal{O}| \alpha\rangle$. Assim temos que:

$$
\sigma_{\text {tot }}^{i N}=\left\langle\sigma_{\alpha}\right\rangle
$$

Pela definição, a seção de choque difrativa em $t=0$ é:

$$
\begin{aligned}
\left.\frac{d \sigma_{i N}^{D}}{d t}\right|_{t=0} & =\frac{1}{16 \pi s^{2}} \sum_{k \neq i} \mathcal{D}_{i k}^{2} \\
& =\frac{1}{16 \pi s^{2}}\left(\sum_{k} \mathcal{D}_{i k}^{2}-\mathcal{D}_{i i}^{2}\right)
\end{aligned}
$$

Observando que:

$$
\begin{aligned}
D_{i k} & =\langle k|D| i\rangle \\
\sum_{k} D_{i k}^{2} & =\sum_{k}\langle k|D| i\rangle^{*}\langle k|D| i\rangle=\sum_{k}\langle i|D| k\rangle\langle k|D| i\rangle=\left\langle i\left|D^{2}\right| i\right\rangle
\end{aligned}
$$

onde usamos a completeza do estado $|k\rangle$, temos:

$$
\left.\frac{d \sigma_{i N}^{D}}{d t}\right|_{t=0}=\frac{1}{16 \pi s^{2}}\left(\left\langle i\left|\mathcal{D}^{2}\right| i\right\rangle-\langle i|\mathcal{D}| i\rangle^{2}\right)
$$


Expandindo $|i\rangle$ como: $|i\rangle=\sum_{\alpha} c_{i \alpha}|\alpha\rangle$, teremos:

$$
\left.\frac{d \sigma_{i N}^{D}}{d t}\right|_{t=0}=\frac{1}{16 \pi}\left(\left\langle\sigma_{\alpha}^{2}\right\rangle-\left\langle\sigma_{\alpha}\right\rangle^{2}\right)
$$

Agora aplicaremos o formalismo descrito acima ao caso do espalhamento inelástico profundo difrativo (DDIS) [3, 5].

No DDIS, os auto-estados difrativos são os dipolos de cor $q \bar{q}$. Assim o estado $|\alpha\rangle$ definido acima será identificado com um estado de Fock $q \bar{q}$ (o estado no qual o fóton virtual, $\gamma^{*}$, flutua antes de encontrar o alvo). A seção de choque $\sigma_{\alpha}$ será substituída pela seção de choque de espalhamento dipolo-próton $\sigma_{\text {dip }}(x, r)$. Assim podemos escrever:

$$
\left.\frac{d \sigma_{L, T}^{D}}{d t}\right|_{t=0}=\frac{1}{16 \pi}\left(\left\langle\sigma_{d i p}^{2}(x, r)\right\rangle_{L, T}-\left\langle\sigma_{d i p}(x, r)\right\rangle_{L, T}^{2}\right)
$$

onde

$$
\left\langle\sigma_{d i p}(x, r)\right\rangle_{L, T} \equiv \int_{0}^{1} d z \int d^{2} \vec{r}\left|\Psi_{L, T}(z, r)\right|^{2} \sigma_{d i p}(x, r)
$$

Uma vez que $\left\langle\sigma_{d i p}(x, r)\right\rangle_{L, T} \equiv \sigma_{L, T}^{\gamma^{*} p}=\mathcal{O}\left(\alpha_{e m}\right)$, podemos desprezar o termo $\left\langle\sigma_{d i p}(x, r)\right\rangle^{2}$, e assim obtemos

$$
\left.\frac{d \sigma_{L, T}^{D}}{d t}\right|_{t=0}=\frac{1}{16 \pi}\left\langle\sigma_{d i p}^{2}(x, r)\right\rangle_{L, T}=\frac{1}{16 \pi} \int_{0}^{1} d z \int d^{2} \vec{r}\left|\Psi_{L, T}(z, r)\right|^{2} \sigma_{d i p}^{2}(x, r)
$$

A seção de choque difrativa é finalmente dada por

$$
\begin{aligned}
\sigma_{d i f f} & =\int_{-\infty}^{0} d t \frac{d \sigma}{d t}=\left.\int_{-\infty}^{0} d t e^{-B_{D} t} \frac{d \sigma}{d t}\right|_{t=0} \\
& =\frac{1}{16 \pi B_{D}} \int d z \int d^{2} r\left|\Psi_{L, T}(z, r)\right|^{2} \sigma_{d i p}^{2}(x, r)
\end{aligned}
$$




\section{Capítulo 3}

\section{Saturação de partons e as equações não lineares de evolução}

\subsection{Introdução}

Conforme discutido no capítulo 1, em espalhamento elétron-próton a altas energias e altos momentos transferidos (espalhamento inelástico profundo - DIS) podemos sondar o interior do próton e determinar as propriedades de seus constituintes. Um resultado surpreendente deste estudo é que a função de distribuição dos glúons $x g\left(x, Q^{2}\right)$, ou seja, o número de glúons observados no interior do próton cresce à medida em que aumentamos a energia do projétil e/ou a virtualidade $Q^{2}$ do fóton emitido pelo elétron. A figura 3.1 mostra a distribuição de glúons como função de $x$ para alguns valores fixos de $Q^{2}$ extraída pela colaboração ZEUS a partir de seus resultados experimentais. O crescimento do número de glúons pode ser entendido como um efeito cascata, onde os próprios glúons se dividem em dois ou três, além de serem também emitidos pelos quarks [3].

Em 1983, Gribov, Levin e Ryskin [30] observaram que em energias muito altas o número de glúons seria tão grande que o processo de recombinação $g+g \rightarrow g$ passaria a ser importante nas equações de evolução. Este processo reduz o crescimento da 


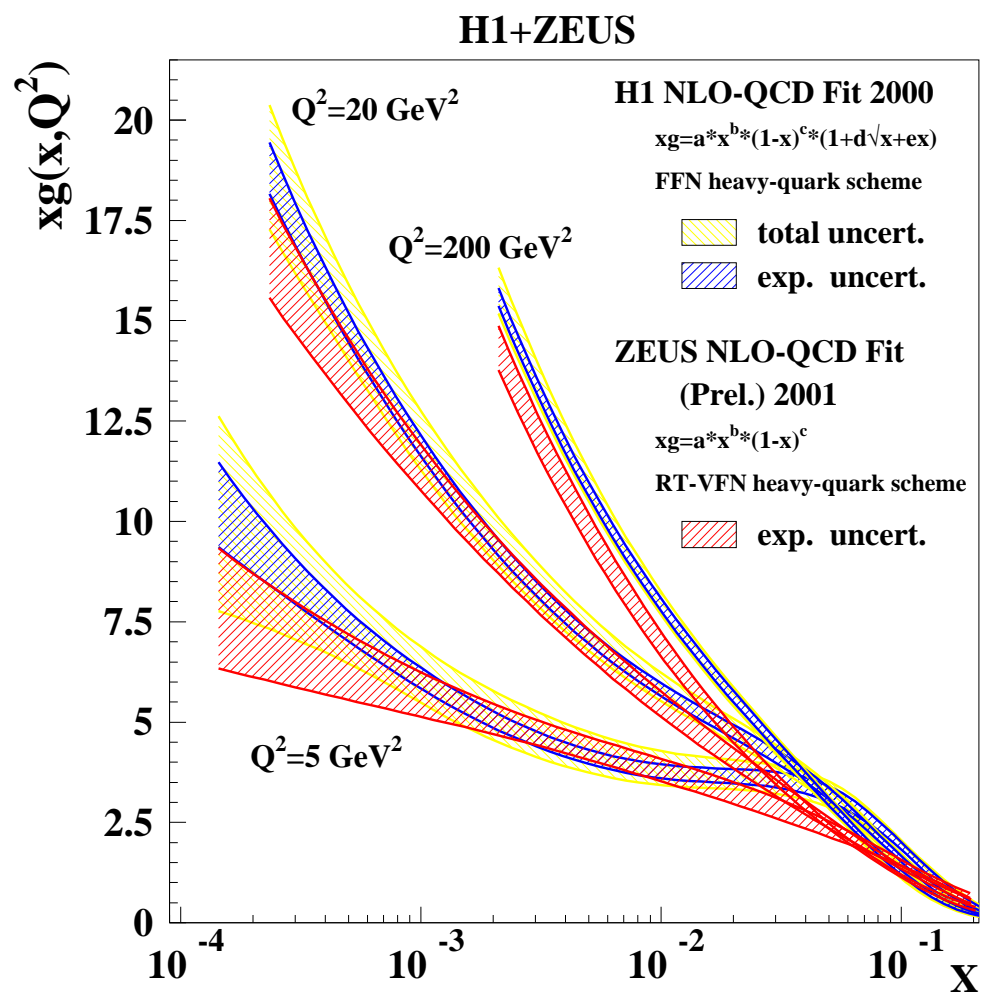

Figura 3.1: Resultados de ZEUS para a distribuição de glúons.

densidade de glúons produzindo um efeito que foi chamado de "saturação perturbativa de partons". Em [30] foi proposta uma equação de evolução não-linear, na dupla aproximação logarítmica (DLLA), na qual $\ln (1 / x)>>1$ e $\ln \left(Q^{2}\right)>>1$, que leva em conta o efeito da recombinação de glúons:

$$
Q^{2} \frac{\partial^{2} x g\left(x, Q^{2}\right)}{\partial \ln (1 / x) \partial Q^{2}}=\frac{\alpha_{S} N_{c}}{\pi} x g\left(x, Q^{2}\right)-\frac{4 \alpha_{S}^{2} N_{c}}{3 C_{F} R^{2}} \frac{1}{Q^{2}}\left[x g\left(x, Q^{2}\right)\right]^{2} .
$$

Observando o sinal negativo do termo não-linear, vemos que o crescimento acentuado de $x g\left(x, Q^{2}\right)$ é atenuado por este termo. Esta equação também implica que em um determinado momento a quantidade de glúons vai parar de crescer. Isto ocorrerá quando os termos não-linear e linear se tornarem idênticos, ou seja, quando $Q^{2} \equiv Q_{s}^{2}$ :

$$
Q_{s}^{2}=\frac{4 \pi \alpha_{s}}{3 C_{F} R^{2}} x g\left(x, Q^{2}\right)
$$


Esta escala é denominada "escala de saturação". O regime caracterizado por $Q^{2}>Q_{s}^{2}$ é o regime linear, enquanto para $Q^{2}<Q_{s}^{2}$ temos o regime não-linear ou de saturação.

O trabalho pioneiro de Gribov, Levin e Ryskin gerou uma longa série de trabalhos cujo objetivo era desenvolver uma teoria da saturação. Uma teoria efetiva da QCD a altas energias e pequenos $x$ é o chamado "Color Glass Condensate" (CGC) [11], que leva às equações de evolução JIMWLK [31]. Uma teoria semelhante, mas com aproximações diferentes, foi desenvolvida por Balitsky e depois por Kovchegov. Dela é possível chegar à equação de evolução chamada de Balitsky-Kovchegov (BK). Estas equações mencionadas são muito complicadas e mesmo a mais simples delas (BK) só pode ser resolvida numericamente.

O Condensado de Vidro de Cor (Color Glass Condensate - CGC) é formado por um sistema de alta densidade hadrônica, que controla as interações em QCD a altas energias (pequenos valores da variável $x$ de Bjorken) [3]. O CGC é colorido por ser composto de glúons, os quais carregam carga de "cor"; ele é um vidro, pois sua dinâmica interna está congelada. Sua escala de tempo natural é muito maior do que a escala de tempo do espalhamento em altas energias. E ele é um condensado por ser caracterizado por um grande número de ocupação e fortes campos coloridos clássicos. A alta densidade e a liberdade assintótica implicam que o CGC é fracamente acoplado, o que permitiu o desenvolvimento de uma teoria efetiva.

Quando comparamos a equação $(1,3.26)$, que é a equação de DGLAP para a distribuição de glúons, com a equação $(3,1.1)$, vemos claramente que a equação $(1,3.26)$ não incorpora a recombinação de glúons e dessa forma o número de glúons tende a crescer continuamente. Como a equação $(1,3.26)$ não tem a dependência quadrática em $g(x)$ (que observamos em $(3,1.1)$ ) ela é chamada de equação linear de evolução.

A figura 3.2 mostra um mapa simbólico das equações de evolução da QCD. Nele podemos ver uma representação pictórica de como ocorre a evolução em $Q^{2}$ da distribuição de partons, dada pela equação de DGLAP. Nesta equação fixamos $x$ e evoluímos em $Q^{2}$. Assim, conforme aumentamos a nossa resolução $Q^{2}$ o sistema se torna mais diluído, pois apesar de o número de glúons crescer a área de cada um deles se 


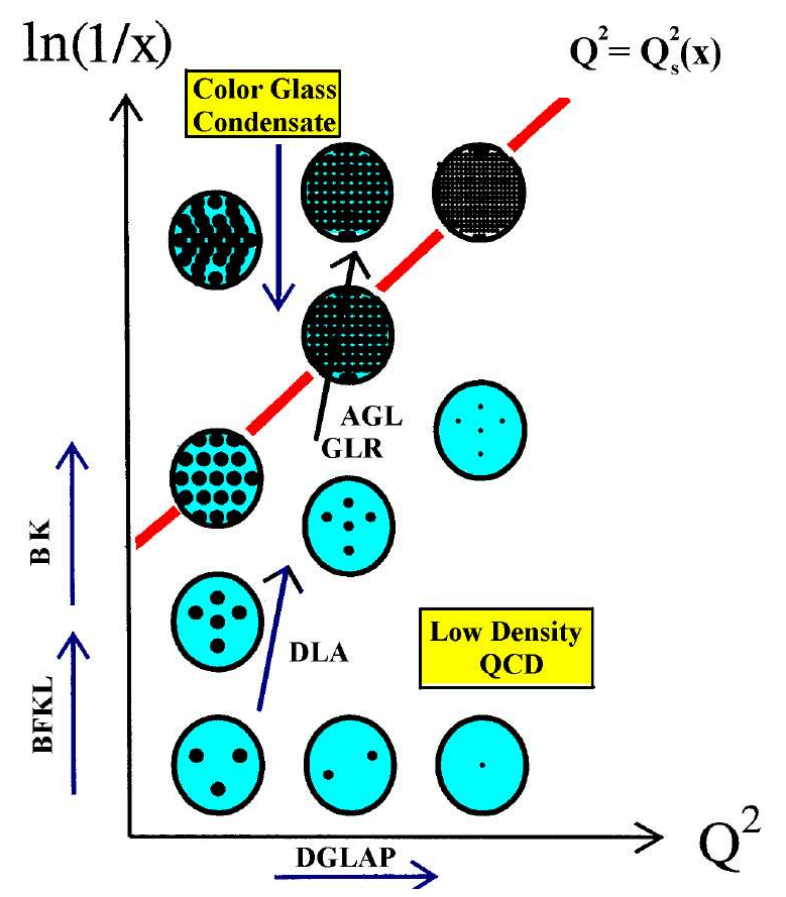

Figura 3.2: Mapa da evolução da QCD. (Extraído de [4]).

torna menor. Nesta figura vemos também uma imagem pictórica de como a equação de BFKL (que conforme veremos mais adiante é uma aproximação da equação de BK) descreve a evolução do sistema com a energia, ou seja, com $1 / x$. Na BFKL o momento transferido $\left(Q^{2}\right)$ é que é fixado e as funções de distribuição são evoluídas em $x$. Desse modo, conforme aumentamos a energia, o número de partons dentro do hádron aumenta, mas a área de cada um deles continua sempre a mesma, já que a resolução (que depende de $Q^{2}$ ) continua a mesma. Nesta visão é fácil perceber que a saturação ocorre quando toda a área do hádron for ocupada pelo crescente número de glúons. Nesta figura também é mostrada uma representação da evolução resultante da equação de Ayala, Gay e Levin (AGL), que é uma equação de evolução não-linear [32, 33].

A linha " $Q s$ " que aparece na figura 3.2 separa o regime linear do não linear. A escala de saturação $Q_{s}$ pode ser entendida usando argumentos geométricos [3, 34]. Um fóton de virtualidade $Q^{2}$ consegue resolver uma distância $r$ dentro do alvo da ordem de (veja a figura 3.3)

$$
r \propto \frac{1}{\sqrt{Q^{2}}} .
$$




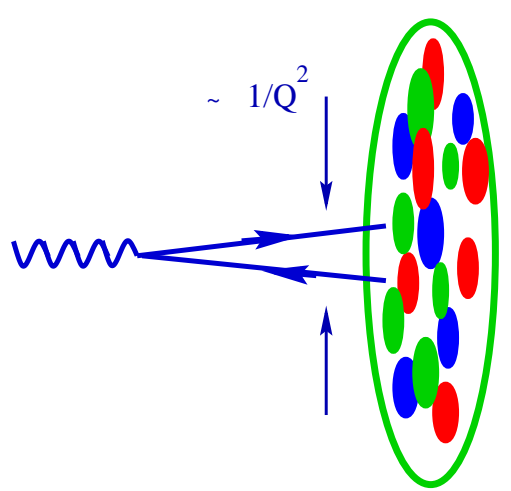

Figura 3.3: Resolução em termos da virtualidade $Q^{2}$ para um fóton sondando o alvo.

A partir dessa relação vemos que, quando aumentamos $Q^{2}$ o dipolo de cor torna-se mais compacto (a distância $r$ entre o quark e o antiquark torna-se menor) e assim consegue resolver objetos menores presentes no alvo (hádron), por exemplo, consegue "enxergar" os glúons. Consequentemente esse dipolo interage com um glúon de área transversal igual à sua. Assim, cada glúon irá ocupar uma área transversal da ordem de $\pi / Q^{2}$, e a seção de choque dipolo-glúon será aproximadamente

$$
\sigma \sim \alpha_{s}\left(Q^{2}\right) \frac{\pi}{Q^{2}}
$$

A área transversal do alvo (hádron) é igual a

$$
S_{A} \sim \pi R_{A}^{2}
$$

sendo $R_{A}$ o raio do alvo nuclear. Quando a soma das áreas de todos os glúons for igual à área do alvo, ou seja

$$
S_{A}=N_{A} \sigma \rightarrow N_{A} \sim \frac{S_{A}}{\sigma} \sim \frac{R_{A}^{2}}{\alpha_{s}} Q^{2}
$$

os glúons começam a sobrepor-se uns aos outros e o processo de recombinação torna-se relevante, inibindo o rápido crescimento da densidade de glúons. A partir da condição 
$(3,1.5)$ a escala de saturação fica definida como:

$$
Q_{s}^{2} \sim \alpha_{s} \frac{N_{A}}{R_{A}^{2}} \sim \frac{\alpha_{s}}{R_{A}^{2}} A x g\left(x, Q^{2}\right) \sim \alpha_{s} A^{1 / 3} x g\left(x, Q^{2}\right)
$$

onde $A$ é o número de massa.

\subsubsection{Evidências experimentais de saturação}

Em 1999 alguns autores [35] passaram a afirmar que, no HERA, já estariam sendo observados alguns efeitos da saturação. À partir de 2000, com a entrada em operação do Relativistic Heavy Ion Collider (RHIC) no Brookhaven National Laboratory (BNL), a procura do CGC tornou-se ainda mais intensa [3]. Surgiram inúmeros trabalhos propondo novos observáveis que pudessem revelar esta componente de alta densidade da função de onda dos hádrons. O CGC é, às vezes, chamado de "um novo estado da matéria". Em 2004, os resultados do RHIC obtidos em colisões dêuteron-ouro levaram muitas pessoas a acreditar que o CGC tinha sido inequivocamente observado. Porém mais tarde a certeza desta descoberta foi questionada.

No artigo de revisão de Larry McLerran [36], de 2004, as seguintes evidências experimentais do CGC são mencionadas:

1. "Scaling"geométrico da seção de choque fóton-próton medida no HERA, caracterizado pela dependência da seção de choque na variável $\tau=r Q_{s}$.

2. Comportamento da função de estrutura $F_{2}$ medida em DIS no HERA.

3. Comportamento da razão entre as seções de choque difrativa e total medidas no HERA.

4. Comportamento da seção de choque de fotoprodução do méson $\rho$ medida no HERA.

5. Comportamento da multiplicidade de partículas carregadas medidas na região central no RHIC. 
6. Comportamento da distribuição de momento transversal de partículas com grande $p_{T}$ e grande rapidez em colisões d-Au no RHIC.

Mais tarde surgiram trabalhos mostrando que estes fenômenos também poderiam ser descritos sem saturação [37]. No entanto, como foi argumentado em [36], o CGC é uma teoria robusta, baseada em primeiros princípios da QCD e fornece uma explicação global para fenômenos muito diferentes entre si.

A conclusão é que ainda não há uma resposta definitiva sobre a existência ou não do CGC, e a busca por sua "assinatura" deve continuar. Conclusões exatas não podem ser tiradas atualmente devido principalmente ao pequeno valor da escala de saturação no alcance cinemático do HERA. Há uma expectativa de que no LHC (Large Hadron Collider) e nos futuros colisores $e A$ será mais fácil observar os efeitos da saturação. Na figura 3.4 podemos ver um exemplo de como as previsões do CGC diferem daquelas feitas com o uso da solução da equação de BFKL na região de pequeno $x$ e pequeno $Q^{2}$, fazendo com que $F_{2}$ cresça muito mais suavemente do que a previsão da dinâmica linear. Vemos que os efeitos associados ao CGC funcionam como um atenuador de tendências que já existem, ou seja, ele afeta os observáveis apenas quantitativamente.

\subsection{A equação de Balitsky-Kovchegov}

A equação BK $[3,39]$ descreve a evolução em rapidez $Y=\ln \left(s / s_{0}\right)=\ln \left(x_{0} / x\right)$ da amplitude de probabilidade de espalhamento $N(\vec{x}, \vec{y}, Y)$ de um dipolo $q \bar{q}$ com o alvo hadrônico, onde $\vec{x}(\vec{y})$ é a posição do quark $q$ (anti-quark $\bar{q}$ ) no espaço transversal com respeito ao centro do alvo. Definimos (Veja figura 3.5.)

$$
\begin{aligned}
\vec{r} & =\vec{x}-\vec{y}, \\
\overrightarrow{r_{1}} & =\vec{x}-\vec{z}, \\
\overrightarrow{r_{2}} & =\vec{y}-\vec{z} \\
\vec{b} & =\frac{\vec{x}+\vec{y}}{2} .
\end{aligned}
$$




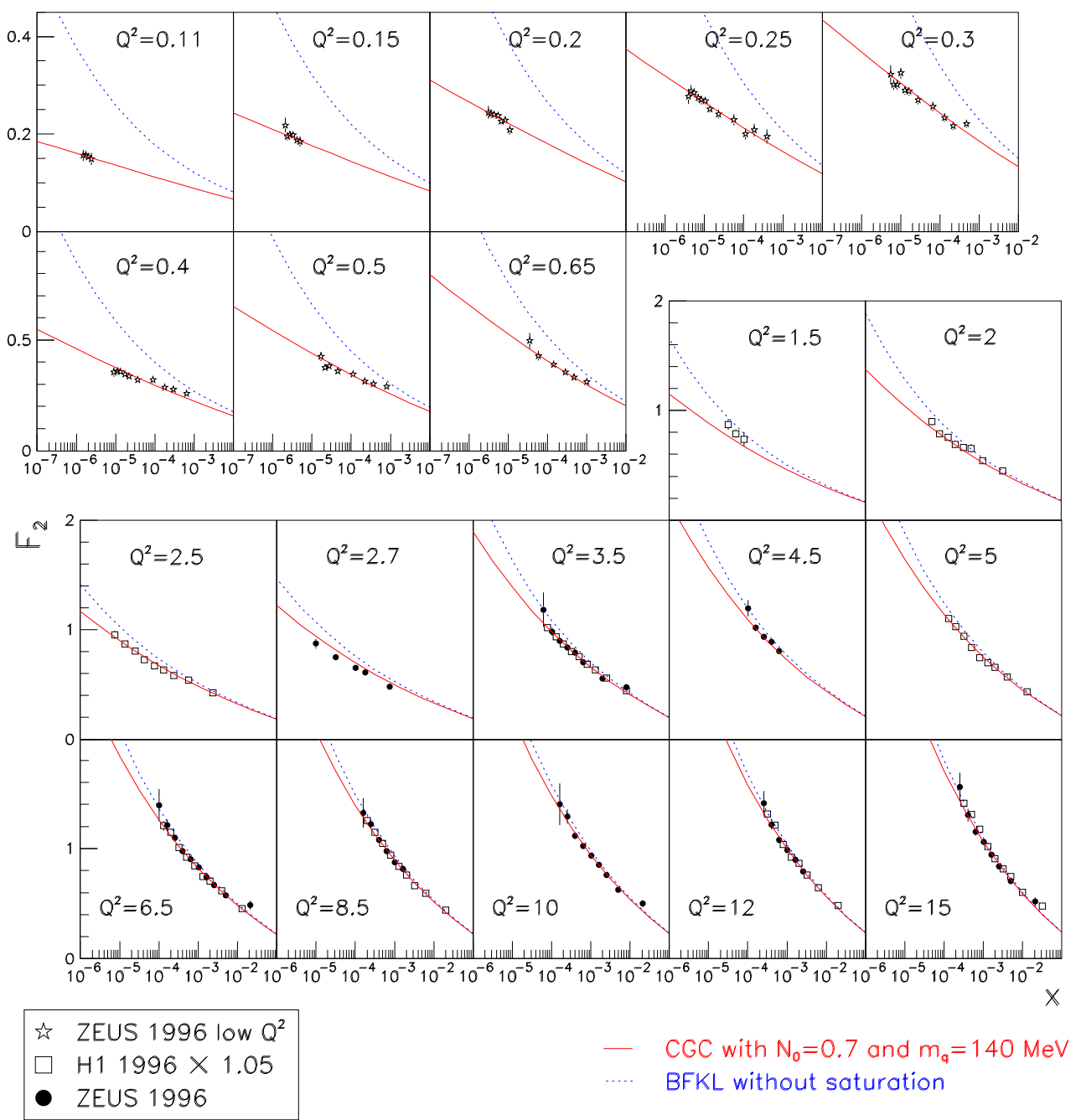

Figura 3.4: As funções de estrutura $F_{2}$ (Extraído de [38]).

Se desprezarmos a dependência no parâmetro de impacto (justificada quando o alvo homogêneo tem raio muito maior do que qualquer tamanho de dipolo considerado), a equação BK fica [3] $(r \equiv|\vec{r}|)$

$$
\begin{aligned}
\frac{\partial N(r, Y)}{\partial Y}= & \int \frac{d^{2} z}{2 \pi} K\left(\vec{r}, \overrightarrow{r_{1}}, \overrightarrow{r_{2}}\right)\left[N\left(r_{1}, Y\right)+N\left(r_{2}, Y\right)\right. \\
& \left.-N(r, Y)-N\left(r_{1}, Y\right) N\left(r_{2}, Y\right)\right]
\end{aligned}
$$




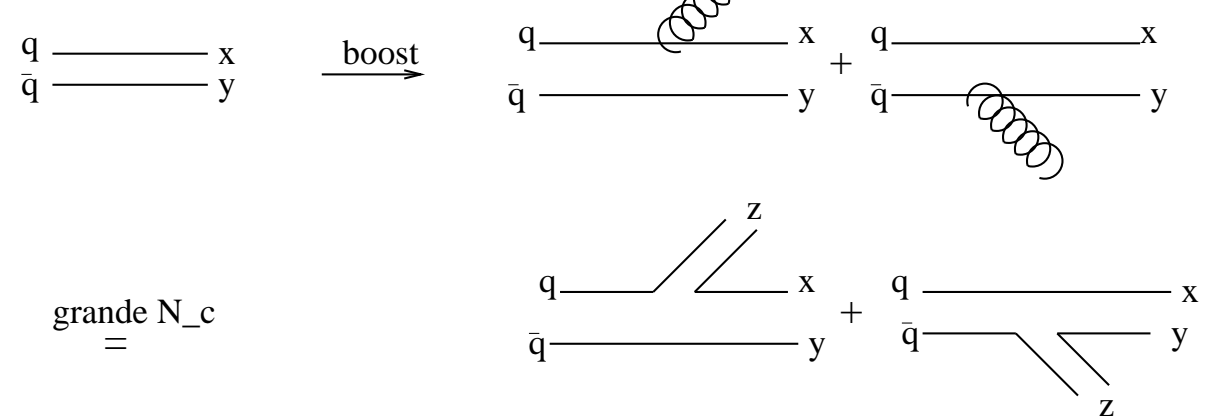

Figura 3.5: Diagrama para a emissão de um glúon na evolução de um dipolo e seu limite de $N_{c} \rightarrow \infty$.

onde o "kernel" BFKL é

$$
\begin{aligned}
K\left(\vec{r}, \overrightarrow{r_{1}}, \overrightarrow{r_{2}}\right) & =\bar{\alpha}_{s} \frac{r^{2}}{r_{1}^{2} r_{2}^{2}} \\
\bar{\alpha}_{s} & =\frac{\alpha_{s} N_{c}}{\pi} .
\end{aligned}
$$

A constante de acoplamento é fixa.

A equação BK tem a seguinte interpretação probabilística (figura 3.5): quando evoluído em rapidez (ou, em outras palavras, quando observado com uma energia maior) o dipolo pai com finais localizados em $\vec{x}$ e $\vec{y}$ emite um glúon, o que corresponde, no limite de grande $N_{c}$, a dois dipolos com finais $(\vec{x}, \vec{z})$ e $(\vec{z}, \vec{y})$. A probabilidade da emissão é dada pelo kernel BFKL $(3,2.8)$ e é multiplicada pela probabilidade de espalhamento dos novos dipolos menos a probabilidade de espalhamento do dipolo pai. O termo não linear é subtraído para evitar a dupla contagem. É este termo não linear que contrasta com a equação BFKL, sendo responsável pelo crescimento limitado da amplitude em rapidez. A equação BK assegura unitariedade, $|N(r, Y)| \leq 1$, localmente na configuração de espaço transversal. Isto é garantido, pois para $N(r, Y)=1$ a derivada com respeito a $Y$ em $(3,2.7)$ não pode ser positiva. 


\subsubsection{A equação de BK no limite de pequeno $x$}

No limite de $x \rightarrow 0, Y \rightarrow \infty$, as soluções da equação BK exibem o fenômeno de scaling geométrico [3, 40], ou seja, as soluções não são mais funções das variáveis $r$ e $Y$ separadamente, mas dependem de uma única variável:

$$
\tau \equiv r Q_{s}(Y)
$$

Nesta região $Q_{s}(Y) \gg \Lambda_{Q C D}$ a equação BK $(3,2.7)$, para constante de acoplamento fixa, pode ser escrita em termos das variáveis $\vec{\tau}=Q_{s} \vec{r}, \vec{\tau}_{1}=Q_{s} \vec{r}_{1}$ e $\vec{\tau}_{2}=Q_{s} \vec{r}_{2}$. A dependência em $Y$ de $N(r, Y)$ está contida em $Q_{s}(Y), N(r, Y) \equiv N(\tau)$. Reescrevendo a derivada do lado esquerdo de $(3,2.7)$, temos

$$
\frac{\partial N(\tau)}{\partial Y}=\frac{\partial N}{\partial \tau} \frac{\partial \tau}{\partial Y}=\frac{\partial Q_{s}(Y)}{\partial Y} r \frac{\partial N}{\partial \tau}=\frac{\partial \ln \left[Q_{s}^{2}(Y) / \Lambda_{Q C D}^{2}\right]}{\partial Y} r^{2} \frac{\partial N}{\partial r^{2}}
$$

Integrando a equação acima em $d^{2} r / r^{2}$, temos

$$
\int \frac{d^{2} r}{r^{2}} \frac{\partial N(\tau)}{\partial Y}=\pi \frac{\partial \ln \left[Q_{s}^{2}(Y) / \Lambda_{Q C D}^{2}\right]}{\partial Y}[N(\infty)-N(0)]=\pi \frac{\partial \ln \left[Q_{s}^{2}(Y) / \Lambda_{Q C D}^{2}\right]}{\partial Y}
$$

onde usamos as condições de contorno $N(\infty)=1$ e $N(0)=0$. Fazendo a mesma integração do lado direito de $(3,2.7)$ e lembrando que

$$
\frac{d^{2} r}{r^{2}}=\frac{d^{2} \tau}{\tau^{2}}
$$

temos que o lado direito de $(3,2.7)$ será:

$$
\int \frac{d^{2} \tau d^{2} \tau_{1}}{2 \pi} \bar{\alpha}_{s} \frac{1}{\tau_{1}^{2} \tau_{2}^{2}}\left[N\left(\tau_{1}\right)+N\left(\tau_{2}\right)-N(\tau)-N\left(\tau_{1}\right) N\left(\tau_{2}\right)\right]
$$


Igualando $(3,2.11)$ com $(3,2.13)$, temos:

$$
\frac{\partial \ln \left[Q_{s}^{2}(Y) / \Lambda_{Q C D}^{2}\right]}{\partial Y}=\bar{\alpha}_{s} \int \frac{d^{2} \tau d^{2} \tau_{1}}{2 \pi^{2}} \frac{1}{\tau_{1}^{2} \tau_{2}^{2}}\left[N\left(\tau_{1}\right)+N\left(\tau_{2}\right)-N(\tau)-N\left(\tau_{1}\right) N\left(\tau_{2}\right)\right]
$$

O integrando é independente de $Y$, então

$$
\frac{\partial \ln \left[Q_{s}^{2}(Y) / \Lambda_{Q C D}^{2}\right]}{\partial Y}=d \bar{\alpha}_{s}
$$

onde $d$ é uma constante. Resolvendo para $Q_{s}$ temos

$$
Q_{s}^{2}=\Lambda_{Q C D}^{2} \exp \left(d \bar{\alpha}_{s} Y\right)
$$

A equação acima é a forma da escala de saturação dependente da rapidez para o caso da constante de acoplamento fixa.

Lembrando que $Y=\ln \left(s / s_{0}\right)$ a expressão acima se transforma em:

$$
\begin{aligned}
& Q_{s}^{2}=\Lambda_{Q C D}^{2} \exp \left(d \bar{\alpha}_{s} \ln \left(s / s_{0}\right)\right) \\
&=\Lambda_{Q C D}^{2}\left(s / s_{0}\right)^{d \bar{\alpha}_{s}}
\end{aligned}
$$

Vemos que a escala de saturação cresce com a energia. Além disso, escrevendo $Y=$ $\ln \left(x_{0} / x\right)$ obtemos uma relação entre a escala de saturação e a variável $x$ de Bjorken:

$$
Q_{s}^{2}=\Lambda_{Q C D}^{2}\left(\frac{x_{0}}{x}\right)^{d \bar{\alpha}_{s}}
$$

A equação acima mostra que a escala de saturação aumenta para valores menores de $x$. A dedução da equação $(3,2.18)$ é uma justificativa teórica da expressão empírica $(3,4.35)$ que será vista adiante. Esta última é conhecida como parametrização de GBW. 


\subsubsection{A equação de BK no limite de grandes dipolos}

Agora voltamos para a equação BK $(3,2.7)$ e a escrevemos em termos de $S$, onde $S$ é o elemento da matriz do espalhamento dipolo-hádron, que está relacionado com a amplitude $N$ de espalhamento dipolo-hádron por:

$$
S=1-N
$$

Assim a equação BK escrita em termos de $S$ fica [3]:

$$
\frac{\partial S(\vec{x}-\vec{y})}{\partial Y}=-\int \frac{d^{2} z}{2 \pi} K(\vec{x}-\vec{y}, \vec{x}-\vec{z}, \vec{y}-\vec{z})[S(\vec{x}-\vec{y})-S(\vec{x}-\vec{z}) S(\vec{y}-\vec{z})]
$$

onde

$$
K(\vec{x}-\vec{y}, \vec{x}-\vec{z}, \vec{y}-\vec{z})=\frac{(\vec{x}-\vec{y})^{2}}{(\vec{x}-\vec{z})^{2}(\vec{y}-\vec{z})^{2}} .
$$

No regime de grandes dipolos (regime de saturação), $r_{\perp} \gg 1 / Q_{s}(Y)$, ou seja, $S\left(r_{\perp}\right) \ll$ 1, a equação acima pode ser linearizada, desprezando o termo quadrático em $S$; isso é válido porque a contribuição dominante vem de $z_{\perp}$ satisfazendo: $1 / Q_{s}(Y) \ll \mid z_{\perp}-$ $x_{\perp} \mid \ll r_{\perp}$. Assim a equação $(3,2.20)$ fica:

$$
\frac{\partial S}{\partial Y}=-\bar{\alpha}_{s} S\left(r_{\perp}\right) \int_{1 / Q_{s}}^{r_{\perp}} \frac{d^{2} z}{2 \pi} \frac{r_{\perp}^{2}}{z_{\perp}^{2}\left(r_{\perp}-z_{\perp}\right)^{2}}
$$

Integrando em $z_{\perp}$ resulta:

$$
\frac{\partial S}{\partial Y} \sim \bar{\alpha}_{s} S\left(r_{\perp}\right) \ln \left(r_{\perp}^{2} Q_{s}^{2}\right)
$$

Definindo a variável de scaling: $\xi \equiv \ln \left[r_{\perp}^{2} Q_{s}^{2}(Y)\right]$ e fazendo

$$
\frac{\partial \xi}{\partial Y}=\frac{\partial \ln \left[r_{\perp}^{2} Q_{s}^{2}(Y)\right]}{\partial Y}=c \bar{\alpha}_{s}
$$

temos

$$
\frac{\partial S}{\partial Y}=\frac{\partial S}{\partial \xi} \frac{\partial \xi}{\partial Y}
$$


Logo,

$$
\frac{\partial S}{\partial \xi} c \bar{\alpha}_{s}=-\bar{\alpha}_{s} \ln \left[r_{\perp}^{2} Q_{s}^{2}(Y)\right] S\left(r_{\perp}\right)
$$

e portanto

$$
\frac{\partial S}{\partial \xi}=-\frac{1}{c} \xi S
$$

Resolvendo a equação acima para $S$ obtemos:

$$
S(\xi)=S_{0} e^{-\xi^{2} / 2 c}
$$

Agora, voltando para a amplitude $N$ de espalhamento dipolo-hádron, temos que

$$
N\left(r_{\perp}, Y\right)=1-S_{0} e^{-l n^{2}\left[r_{\perp}^{2} Q_{s}^{2}(Y)\right] / 2 c},
$$

que é conhecida como lei de Levin-Tuchin [41] e que apresenta scaling geométrico.

\subsection{A equação de BFKL no limite de saturação}

No regime linear a equação BK $(3,2.7)$, depois de algumas manipulações, se reduz a $[3,42]:$

$$
\frac{\partial N\left(r_{\perp}\right)}{\partial Y}=-\bar{\alpha}_{s} \int \frac{d^{2} z}{\pi} \frac{r_{\perp}^{2}}{z_{\perp}^{2}\left(r_{\perp}-z_{\perp}\right)^{2}}\left(N\left(z_{\perp}\right)-\frac{1}{2} N\left(r_{\perp}\right)\right)
$$

que é a equação de BFKL. A solução desta equação para dipolos de tamanho $r_{\perp}$ bem menor do que $1 / Q_{s}$, mas próximo deste valor em escala logarítmica é [42]:

$$
N\left(r_{\perp}\right) \simeq \kappa\left(r_{\perp}^{2} Q_{s}^{2}(Y)\right)^{\gamma_{s}} \exp \left[-\frac{\gamma_{s}^{\prime}}{2 \bar{\alpha}_{s} Y}\left(\ln \frac{1}{r_{\perp}^{2} Q_{s}^{2}(Y)}\right)^{2}\right]
$$

onde definimos $\gamma_{s}^{\prime} \equiv \frac{d \gamma_{0}(\mathcal{R})}{d \mathcal{R}} \mathcal{R}_{s}$ (veja no Apêndice A), e também foi usada a condição de contorno $N\left(r_{\perp}=1 / Q_{s}\right)=1$, ou seja, a existência de um regime de saturação. Alguns detalhes da obtenção desta solução podem ser encontrados nos Apêndices $A$ e $B$. 


\subsection{Modelos fenomenológicos}

A seção de choque de dipolo $\sigma_{\text {dip }}$ contém todas as informações sobre o alvo e sobre a física das interações fortes. No formalismo do condensado de vidro de cor (CGC) [43, 44, 45], $\sigma_{\text {dip }}$ pode ser calculado na aproximação eikonal e é dado por:

$$
\sigma_{d i p}(x, \boldsymbol{r})=2 \int d^{2} \boldsymbol{b} \mathcal{N}(x, \boldsymbol{r}, \boldsymbol{b})
$$

onde $\mathcal{N}$ é a amplitude de espalhamento dipolo-alvo para um dado parâmetro de impacto $\boldsymbol{b}$.

Devido às grandes dificuldades envolvidas em obter uma solução exata da equação de BK (ou da equação de JIMWLK) na rapidez $Y=\ln (1 / x)$, tem sido necessário usar modelos fenomenológicos para $\mathcal{N}$ que apresentem suas principais propriedades [3]. As principais propriedades de $\mathcal{N}$ são:

1. para pequenos dipolos $\left(\boldsymbol{r} \ll 1 / Q_{\mathrm{s}}\right)$ temos que $\mathcal{N}(\boldsymbol{r}) \propto \boldsymbol{r}^{2}$, o que implica que o sistema é fracamente interagente;

2. para grandes dipolos $\left(\boldsymbol{r} \gg 1 / Q_{\mathrm{s}}\right)$ devemos ter $\mathcal{N}(\boldsymbol{r}) \approx 1$, e portanto o sistema é fortemente absorvedor. Esta propriedade está associada à grande densidade de glúons na função de onda hadrônica.

Alguns modelos fenomenológicos assumem também que a dependência de $\mathcal{N}$ no parâmetro de impacto pode ser fatorizada como

$$
\mathcal{N}(x, \boldsymbol{r}, \boldsymbol{b})=\mathcal{N}(x, \boldsymbol{r}) S(\boldsymbol{b})
$$

tal que

$$
\sigma_{d i p}(x, \boldsymbol{r})=\sigma_{0} \mathcal{N}(x, \boldsymbol{r})
$$

com $\sigma_{0}$ sendo um parâmetro livre relacionado à QCD não-perturbativa.

Vários modelos para a seção de choque de dipolo têm sido usados na literatura para 
descrever os dados de HERA. Nesta dissertação, no entanto, estamos interessados em apenas dois deles, que descreveremos à seguir.

\subsubsection{O modelo de GBW}

Em [35] Golec-Biernart e Wüsthoff (GBW) propuseram o seguinte modelo fenomenológico de saturação:

$$
\mathcal{N}(x, \boldsymbol{r})=\left[1-\exp \left(-\frac{\left(Q_{\mathrm{s}}(x) \boldsymbol{r}\right)^{2}}{4}\right)\right]
$$

com

$$
Q_{s}^{2}(x)=Q_{0}^{2}\left(\frac{x_{0}}{\tilde{x}}\right)^{\lambda} \quad ; \quad \tilde{x}=x\left(1+\frac{4 m_{f}^{2}}{Q^{2}}\right)
$$

onde alguns parâmetros foram fixados:

$$
Q_{0}^{2}=1 \mathrm{GeV}^{2} \quad ; \quad m_{u} \approx m_{d} \approx m_{s} \approx 0,14 \mathrm{GeV}
$$

e $\sigma_{0}, \lambda$ e $x_{0}$ foram determinados à partir de um fit aos dados experimentais de HERA. No caso de três sabores de quarks $(\mathrm{u}, \mathrm{d}, \mathrm{s})$ obteve-se:

$$
\sigma_{0}=23,03 m b \quad ; \quad \lambda=0,288 \quad ; \quad x_{0}=3,04 \times 10^{-4}
$$

Olhando para a equação $(3,4.34)$ podemos notar que quando $Q_{s}^{2}(x) \boldsymbol{r}^{2} \ll 1$ o modelo satisfaz a primeira propriedade requerida para $\mathcal{N}$, i.e., a transparência de $\operatorname{cor}(\mathcal{N}(\boldsymbol{r}) \propto$ $\boldsymbol{r}^{2}$ ); e na região $Q_{s}^{2}(x) \boldsymbol{r}^{2}>1$ a exponencial assume valores muito menores do que um, o que implica que para dipolos muito grandes temos $\mathcal{N} \approx 1$.

Embora a parametrização GBW dê uma boa descrição dos dados antigos de HERA isso não ocorre para os novos dados, que têm uma precisão maior. Esta limitação vem do fato de que este modelo falha ao descrever a violação de scaling de Bjorken, e sua forma funcional vem apenas de uma aproximação da QCD não-linear. 


\subsubsection{O modelo b-CGC}

Na referência [38] foi proposto um outro modelo inspirado no CGC para descrever os dados de HERA. Este modelo está baseado na aproximação da equação de BFKL na fronteira do regime de saturação. Assim, o ponto de partida é a equação $(0,0.12)$

$$
\mathcal{N}(x, \boldsymbol{r})=\left[\boldsymbol{r}^{2} Q_{s}^{2}(x)\right]^{\gamma_{s}} \exp \left[-\frac{\ln ^{2}\left(\boldsymbol{r}^{2} Q_{s}^{2}\right)}{2 \beta \bar{\alpha}_{s} Y(x)}\right]
$$

onde $\gamma_{s}$ é o ponto de sela (BFKL) na vizinhança da linha de saturação $Q^{2}=Q_{s}^{2}(x)$ e $\beta=1 / \gamma_{s}^{\prime}$. Neste modelo a normalização da seção de choque de dipolo é dada por $\sigma_{0}=$ $2 \pi R_{p}^{2}$, onde $R_{p}$ é o raio do próton. Além disso a dimensão anômala é definida por $\gamma=$ $1-\gamma_{s}$, e como usual no formalismo de BFKL temos $\bar{\alpha}_{s}=N_{c} \alpha_{s} / \pi$ e $\beta \simeq 28 \zeta(3)$. O fator de difusão quadrático no expoente é responsável pelo aumento da violação de scaling, que por sua vez é essencial para descrever os dados de HERA. Como a amplitude de espalhamento frontal $(3,4.38)$ não inclui uma extrapolação da região de scaling geométrico para a região de saturação, os autores da referência [38] propuseram uma parametrização para $\mathcal{N}(x, \boldsymbol{r})$ que interpola suavemente entre os limites de pequeno e grande dipolo, i.e., entre a solução da equação de BFKL para pequenos dipolos, $\boldsymbol{r} \ll 1 / Q_{s}(x)$, e a Lei de Levin-Tuchin (equação $(3,2.28)$ ) para grandes dipolos, $\boldsymbol{r} \gg$ $1 / Q_{s}(x)$. Assim a amplitude de espalhamento frontal dipolo-alvo foi parametrizada como:

$$
\mathcal{N}(x, \boldsymbol{r})= \begin{cases}\mathcal{N}_{0}\left(\frac{\boldsymbol{r} Q_{s}}{2}\right)^{2\left(\gamma_{s}+\frac{\ln \left(2 / \boldsymbol{r}_{\left.Q_{s}\right)}\right.}{\kappa \lambda Y}\right),} & \text { para } \boldsymbol{r} Q_{s}(x) \leq 2, \\ 1-\exp ^{-a \ln ^{2}\left(b \boldsymbol{r} Q_{s}\right)}, & \text { para } \boldsymbol{r} Q_{s}(x)>2,\end{cases}
$$

Os coeficientes $a$ e $b$ são determinados unicamente pela condição de que $\mathcal{N}(x, r)$ e sua derivada com relação a $r Q_{s}$ sejam contínuas em $r Q_{s}=2$. Chamaremos este modelo de CGC.

O modelo b-CGC, proposto na Ref. [46], é uma modificação do modelo CGC tal que a seção de choque diferencial dipolo-próton deste modelo passa a depender do 
parâmetro de impacto $\bar{b}$. Segundo este modelo temos:

$$
\sigma_{d i p}\left(x, r^{2}\right) \equiv \int d^{2} \bar{b} \frac{d \sigma_{d i p}}{d^{2} \bar{b}} \quad ; \quad \frac{d \sigma_{d i p}}{d^{2} \bar{b}}=2 \mathcal{N}(x, \boldsymbol{r}, \bar{b})
$$

onde

$$
\mathcal{N}(x, \boldsymbol{r}, \bar{b})= \begin{cases}\mathcal{N}_{0}\left(\frac{\boldsymbol{r} Q_{s, p}}{2}\right)^{2\left(\gamma_{s}+\frac{\ln \left(2 / \boldsymbol{r}_{Q s, p)}\right.}{\kappa \lambda Y}\right)}, & \text { para } \boldsymbol{r} Q_{s, p}(x) \leq 2, \\ 1-\exp ^{-a \ln ^{2}\left(b \boldsymbol{r} Q_{s, p}\right)}, & \text { para } \boldsymbol{r} Q_{s, p}(x)>2\end{cases}
$$

Veja que $\mathcal{N}(x, \boldsymbol{r}, \bar{b})$ é dado por uma expressão idêntica àquela de $\mathcal{N}(x, \boldsymbol{r})$ do modelo CGC (equação $(3,4.39)$ ), porém agora temos $Q_{s, p}(x, \bar{b})$ no lugar de $Q_{s}(x)$. No b-CGC a escala de saturação do próton $Q_{s, p}$ é dada por:

$$
Q_{s, p}(x, \bar{b})=\left(\frac{x_{0}}{x}\right)^{\frac{\lambda}{2}}\left[\exp \left(-\frac{\bar{b}^{2}}{2 B_{C G C}}\right)\right]^{\frac{1}{2 \gamma_{s}}}
$$

Os parâmetros do modelo b-CGC foram fitados recentemente para descrever os dados atuais de HERA [47]. Os fatores $\mathcal{N}_{0}$ e $\gamma_{s}$ foram considerados livres. Desta maneira uma boa descrição dos dados de $F_{2}$ foi obtida. O conjunto de parâmetros que utilizamos nesta dissertação é o da segunda linha da tabela II de [47]: $\gamma_{s}=0,46$, $B_{C G C}=7,5 \mathrm{GeV}^{-2}, N_{0}=0,558, x_{0}=1,84 \times 10^{-6}$ e $\lambda=0,119$. 


\section{Capítulo 4}

\section{Funções de estrutura e a distribuição nuclear de glúons}

Conforme mostra a figura 1.6, a distribuição nuclear de glúons ainda é bastante mal determinada. Na seção 1.7 vimos que os observáveis inclusivos $F_{L}$ e $F_{2}^{c}$ são fortemente dependentes da distribuição de glúons. Nosso objetivo é quantificar e determinar a região cinemática onde estes observáveis determinam diretamente o comportamento de $R_{g}$. Para obter conclusões independentes do modelo nós calculamos $R_{L}$ e $R_{C}$, dados respectivamente por $(1,8.59)$ e $(1,8.63)$, usando as quatro parametrizações mencionadas no capítulo 1 e comparamos com as previsões correspondentes para $R_{g}$. Aqui $R_{L}$ e $R_{C}$ são as razões similares a $R_{g}$ para $F_{L}$ e $F_{2}^{c}$, i.e., as razões respectivamente de $F_{L}$ e $F_{2}^{c}$ do nucleon ligado ao núcleo para o nucleon livre. Como a região de pequeno $x$ no eRHIC será sondada em pequeno $Q^{2}$, nós concentramos nossa análise em dois valores característicos de $Q^{2}: Q^{2}=2,5 \mathrm{GeV}^{2}$ e $10 \mathrm{GeV}^{2}$.

Nas figuras 4.1 e 4.2 , olhando para a região de pequeno $x, x<10^{-3}$, vemos que:

- $R_{L}$ praticamente coincide com $R_{g}$ para todas as parametrizações e para os dois valores de $Q^{2}$ considerados. Isto sugere que efeitos de shadowing na distribuição de glúons podem ser facilmente determinados no eRHIC através da medição de $F_{L}$. 


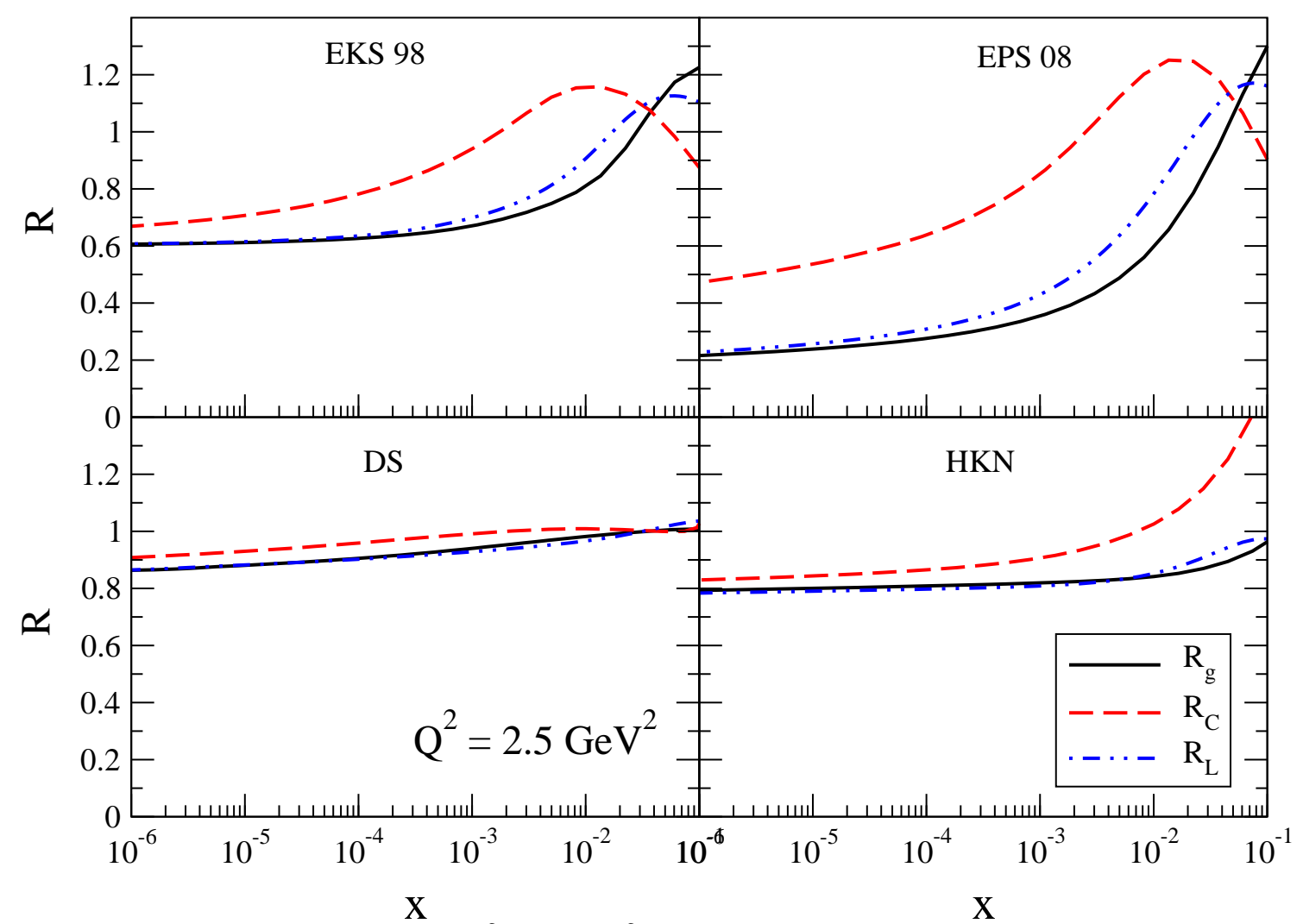

Figura 4.1: Razões $R_{g}, R_{C}$ e $R_{L}, \operatorname{com} Q^{2}=2,5 G e V^{2}$ e $\boldsymbol{A}=\mathbf{2 0 8}$, para as quatro parametrizações nucleares consideradas.

- A razão $R_{C}$ nos dá um limite superior para a magnitude dos efeitos de shadowing. Por exemplo: se for encontrado $R_{C} \approx 0,6$ para $x=10^{-4}$ e $Q^{2}=2,5 \mathrm{GeV}^{2}$, as distribuições nucleares de glúons das parametrizações DS e HKN são muito grandes e deveriam ser modificadas.

- Em $Q^{2}=10 \mathrm{GeV}^{2}$ o comportamento de $R_{C}$ é quase idêntico a $R_{g}$, o que implica que medindo $F_{2}^{c}$ nesta virtualidade nós também podemos determinar os efeitos de shadowing.

Olhando agora para a região $x>10^{-3}$ vemos que:

- Novamente o comportamento de $R_{L}$ é muito próximo do de $R_{g}$. 


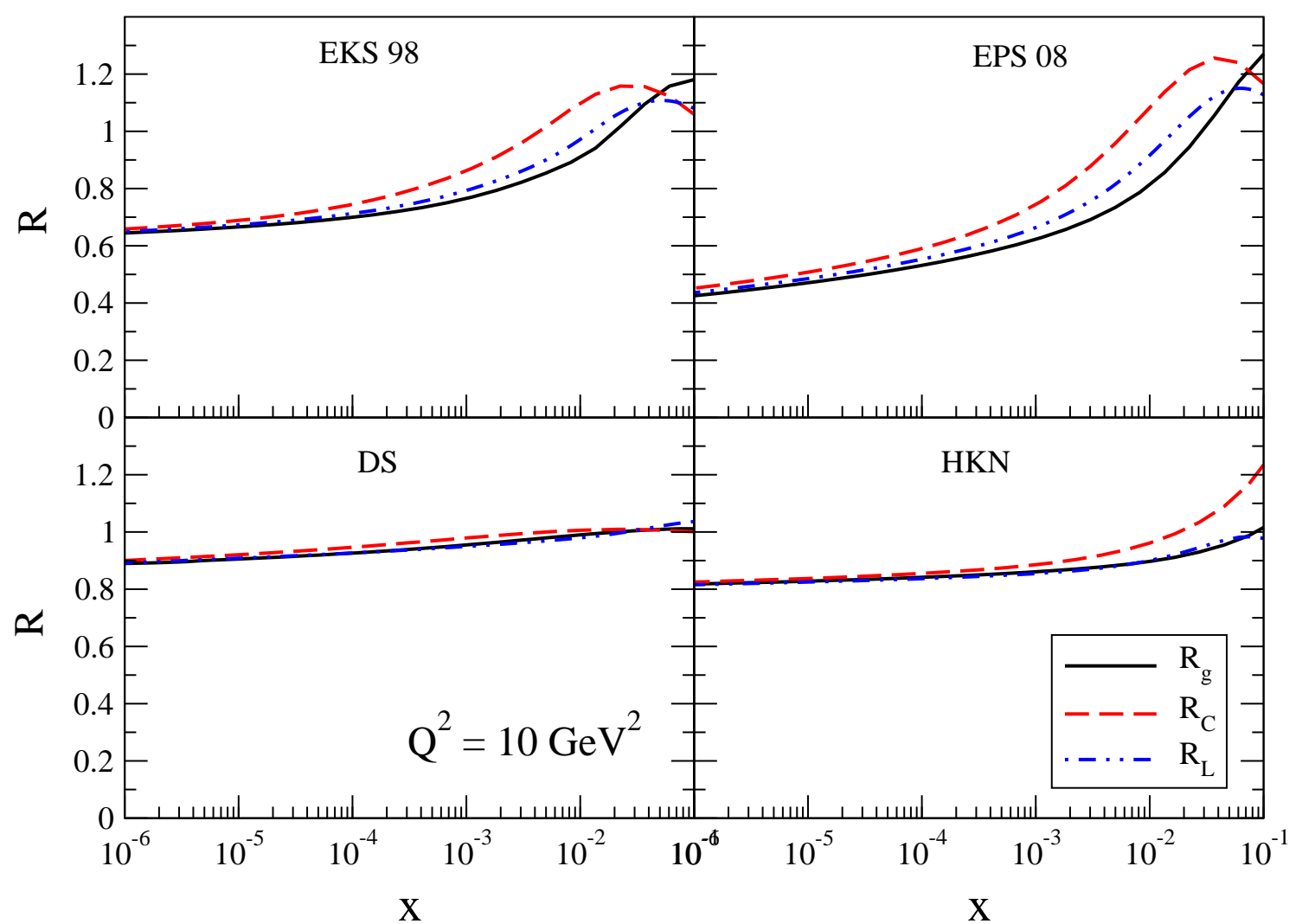

Figura 4.2: Razões $R_{g}, R_{C}$ e $R_{L}, \operatorname{com} Q^{2}=10 G e V^{2}$ e $\boldsymbol{A}=208$, para as quatro parametrizações nucleares consideradas.

- Em particular, a presença de antishadowing em $x g_{A}$, predita por EKS e EPS, implica diretamente num aumento em $F_{L}^{A}$. E a não existência de antishadowing em $x g_{A}$ para $x<10^{-1}$, predita por DS e HKN, implica que não haverá este efeito também em $F_{L}^{A}$.

- O comportamento de $R_{C}$ nesta região cinemática é diferente de $R_{g}$. Entretanto, o comportamento de $R_{C}$ em $x=10^{-2}$ é diretamente associado a $R_{g}$ em $x=10^{-1}$. Em outras palavras, o antishadowing é deslocado em $R_{C}$ por aproximadamente uma ordem de magnitude em $x$. Consequentemente, medindo-se $F_{2}^{c}$ é também possível vincular a existência e magnitude dos efeitos de antishadowing.

Até aqui consideramos apenas $A=208$. Nas figuras 4.3 e 4.4 são mostradas as 


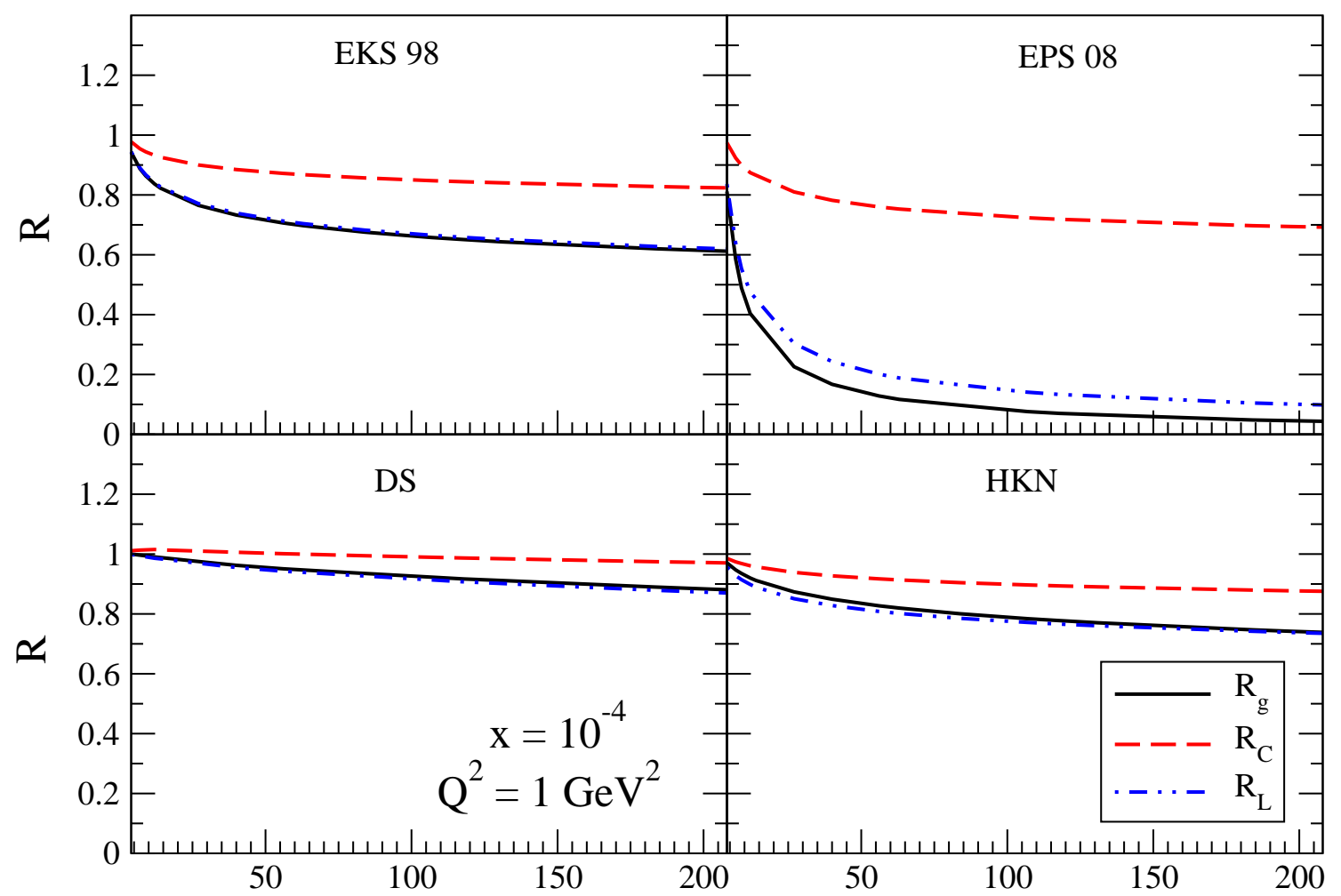

A

A

Figura 4.3: Razões $R_{g}, R_{C}$ e $R_{L}$, com $Q^{2}=1 G e V^{2}$ e $x=10^{-4}$, para as quatro parametrizações nucleares consideradas.

razões $R_{g}, R_{C}$ e $R_{L}$, como funções de $A$, para as quatro parametrizações com duas combinações típicas de $x$ e $Q^{2}$.

Nós observamos uma dependência em $A$ mais forte das razões para os menores valores de $x$ e $Q^{2}$ (Fig. 4.3). Isto está diretamente associado à magnitude e comportamento do efeito de shadowing, que é esperado ser maior nesta região cinemática. Uma característica importante destes gráficos é que eles realçam nossas conclusões anteriores de que em todos os domínios cinemáticos e para diferentes alvos $R_{g}$ aproximadamente coincide com $R_{L}$, que é uma quantidade mensurável.

Com base nestes resultados concluímos que o estudo dos observáveis inclusivos $F_{L}$ e $F_{2}^{c}$ em processos $e A$ no futuro eRHIC é ideal para determinar os efeitos nucleares presentes na distribuição nuclear de glúons, que por sua vez é crucial para 


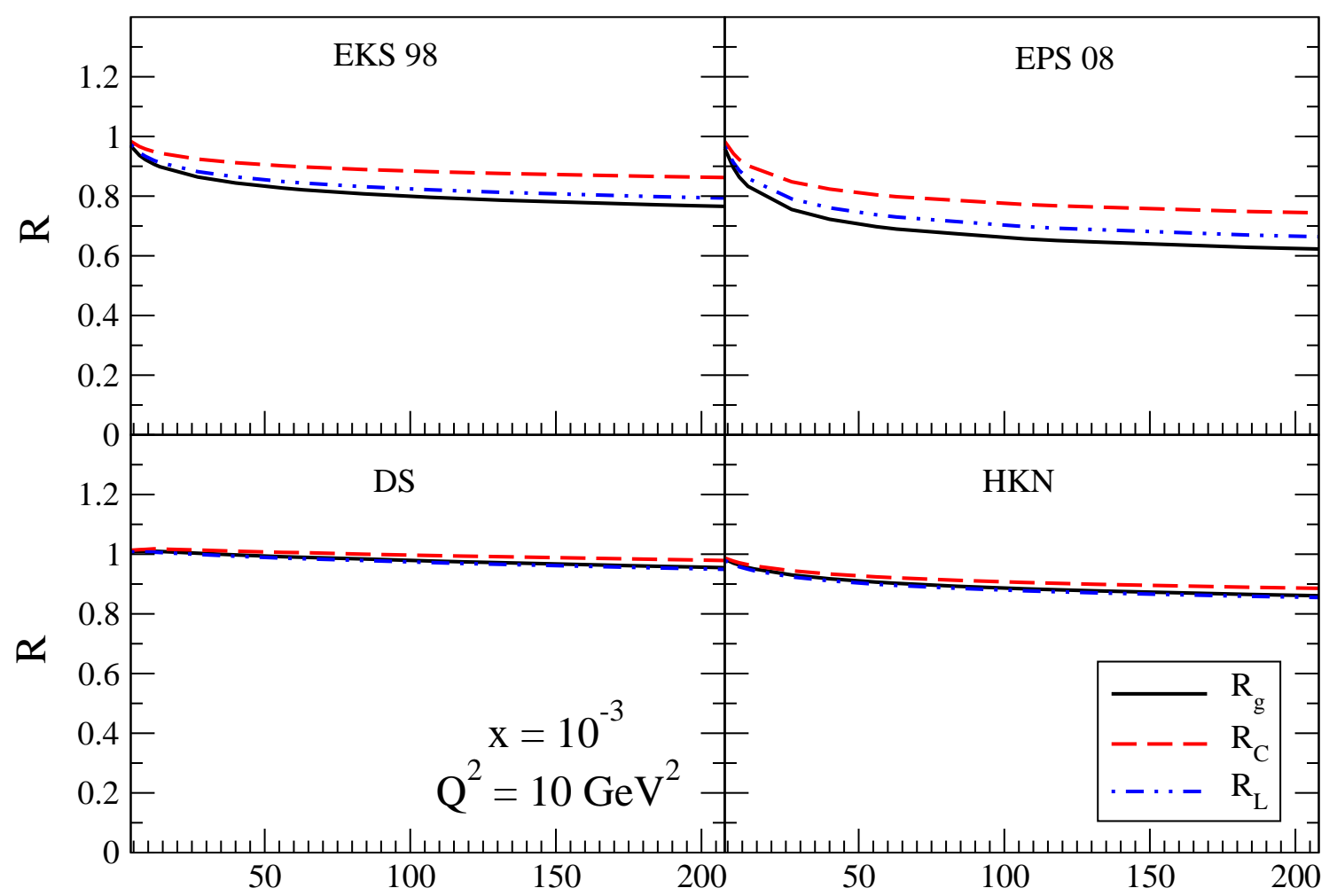

A

A

Figura 4.4: Razões $R_{g}, R_{C}$ e $R_{L}$, com $Q^{2}=10 G e V^{2}$ e $x=10^{-3}$, para as quatro parametrizações nucleares consideradas.

calcular as seções de choque dos processos que serão estudados nos futuros aceleradores. Basicamente nós vemos que medindo estes observáveis nós teremos um acesso direto à distribuição nuclear de glúons, o que permitirá discriminar entre as diferentes parametrizações. 


\section{Capítulo 5}

\section{Efeitos de saturação em colisores $e A$}

\subsection{A generalização nuclear da escala de saturação}

Conforme discutido no capítulo 3 , a escala de saturação $Q_{s}$ cresce com a energia (veja 3,2.16). Uma generalização simples de $Q_{s}$ para o caso de um núcleo de número de massa $A$ pode ser feita pela seguinte relação

$$
Q_{s}^{2}=Q_{s_{0}}^{2} A^{1 / 3}\left(\frac{1}{x}\right)^{\lambda}
$$

onde $\lambda=0,3$ é um parâmetro fixado pelo ajuste de dados ep do HERA, como foi feito na referência [35]. A figura 5.1 mostra um gráfico do plano $A^{1 / 3}$ versus $x$, onde as linhas representam escalas de saturação constantes. Como podemos ver na figura, o núcleo amplia a densidade de glúons e faz com que o regime de saturação seja atingido em energias mais baixas (maiores valores de $x$ ) do que em colisões contra um nucleon. Por isto, processos nos quais um núcleo esteja presente são ideais para testar a física de saturação. 


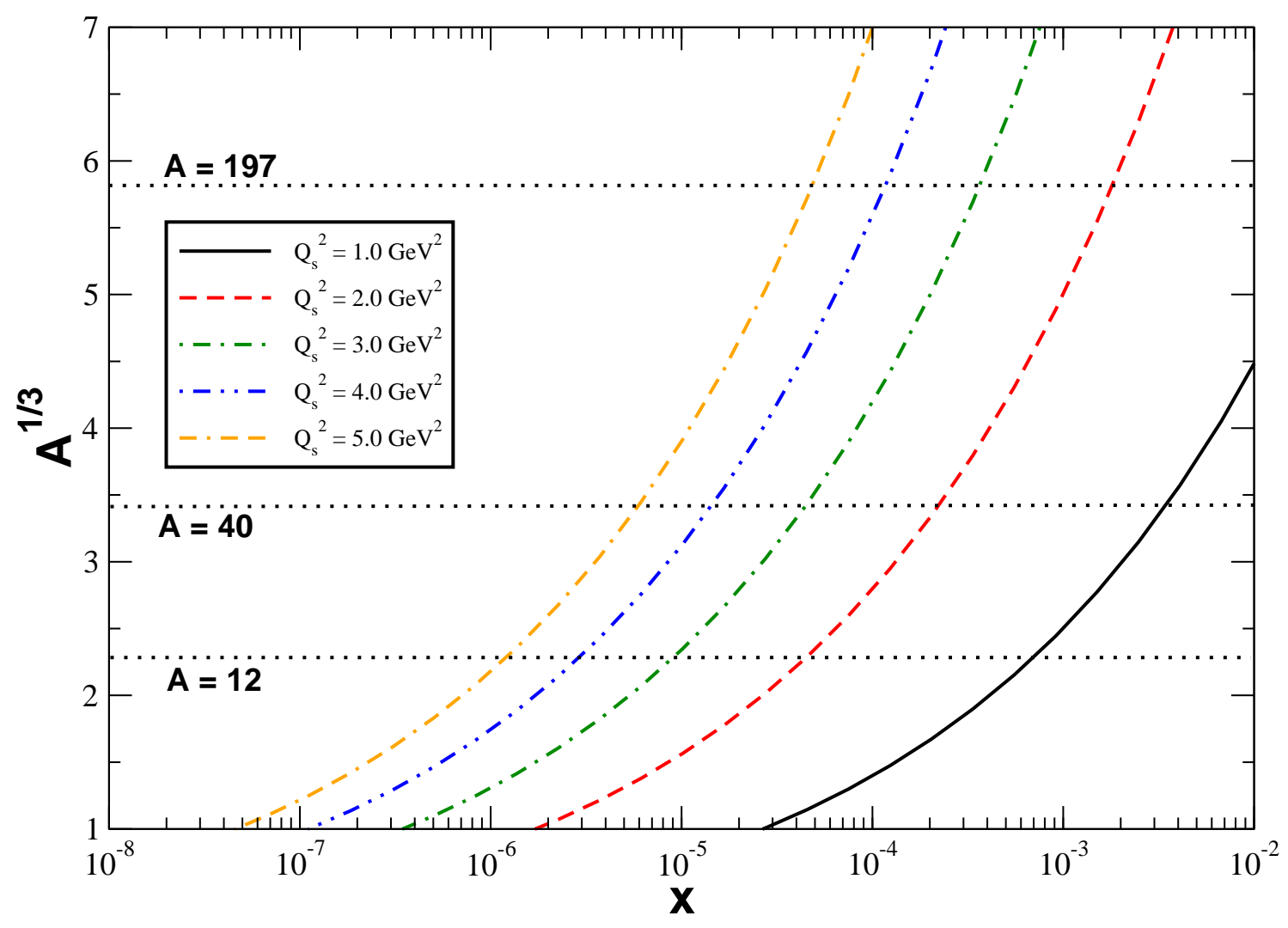

Figura 5.1: Escala de saturação para diferentes valores de $A$ e $x$.

\subsection{Observáveis inclusivos nucleares}

Generalizando os resultados do formalismo de dipolo de cor (discutidos no capítulo 2) para um núcleo $A$, temos que a função de estrutura nuclear $F_{2}^{A}$ é dada por

$$
F_{2}^{A}\left(x, Q^{2}\right)=\frac{Q^{2}}{4 \pi^{2} \alpha_{e m}} \sigma_{t o t}^{A}
$$

com

$$
\sigma_{\text {tot }}^{A}=\sigma_{L}^{A}+\sigma_{T}^{A} \quad ; \quad \sigma_{T, L}^{A}=\int d^{2} r d z\left|\Psi_{T, L}\left(r, z, Q^{2}\right)\right|^{2} \sigma_{d i p}^{A}(x, r)
$$

Agora temos $\sigma_{\text {dip }}^{A}$ (a seção de choque dipolo-núcleo) no lugar de $\sigma_{\text {dip }}$ (a seção de choque dipolo-nucleon). Da mesma forma a função de estrutura longitudinal nuclear é dada 
por:

$$
F_{L}^{A}\left(x, Q^{2}\right)=\frac{Q^{2}}{4 \pi^{2} \alpha_{e m}} \sigma_{L}^{A}
$$

com $\sigma_{L}^{A}$ obtido tomando-se a componente $L$ de $(5,2.3)$. A componente de charme $F_{2}^{c, A}\left(x, Q^{2}\right)$ da função de estrutura nuclear é obtida diretamente da equação $(5,2.2)$ isolando o sabor charme e considerando $m_{c}=1,5 \mathrm{GeV}$.

Vemos que para obtermos os observáveis inclusivos nucleares acima devemos generalizar $\sigma_{\text {dip }}$ para $\sigma_{\text {dip }}^{A}$. Na aproximação eikonal temos:

$$
\sigma_{d i p}^{A}(x, \boldsymbol{r})=2 \int d^{2} \boldsymbol{b} \mathcal{N}^{A}(x, \boldsymbol{r}, \boldsymbol{b})
$$

onde $\mathcal{N}^{A}$ é a amplitude de espalhamento para um dipolo com tamanho $\boldsymbol{r}$ e parâmetro de impacto $\boldsymbol{b}$.

Um modelo fenomenológico que descreve os escassos dados experimentais disponíveis da função de estrutura nuclear e que inclui a dependência no parâmetro de impacto na seção de choque dipolo-núcleo foi proposto há alguns anos na Ref. [48]. Neste modelo a amplitude dipolo-núcleo foi parametrizada como:

$$
\mathcal{N}^{A}(x, r, b)=1-\exp \left[-\frac{1}{2} A T_{A}(b) \sigma_{\text {dip }}\left(x, r^{2}\right)\right]
$$

onde $T_{A}(b)$ é a função de perfil nuclear, que é obtida de uma distribuição de Fermi de 3 parâmetros para a densidade nuclear normalizada a 1 [49]. Em [48] o autor assumiu $\sigma_{\text {dip }}$ como sendo dada pelo modelo de GBW (veja seção 3.5), i.e.,

$$
\sigma_{d i p}(x, r)=\sigma_{0}\left[1-\exp \left(-\frac{\left(Q_{\mathrm{s}}(x) \boldsymbol{r}\right)^{2}}{4}\right)\right]
$$

com

$$
Q_{s}^{2}(x)=Q_{0}^{2}\left(\frac{x_{0}}{\tilde{x}}\right)^{\lambda} \quad ; \quad \tilde{x}=x\left(1+\frac{4 m_{f}^{2}}{Q^{2}}\right)
$$

onde $Q_{0}^{2}=1 \mathrm{GeV}^{2}, m_{u} \approx m_{d} \approx m_{s} \approx 0,14 \mathrm{GeV}, \sigma_{0}=23,03 \mathrm{mb}, \lambda=0,288 \mathrm{e}$ 


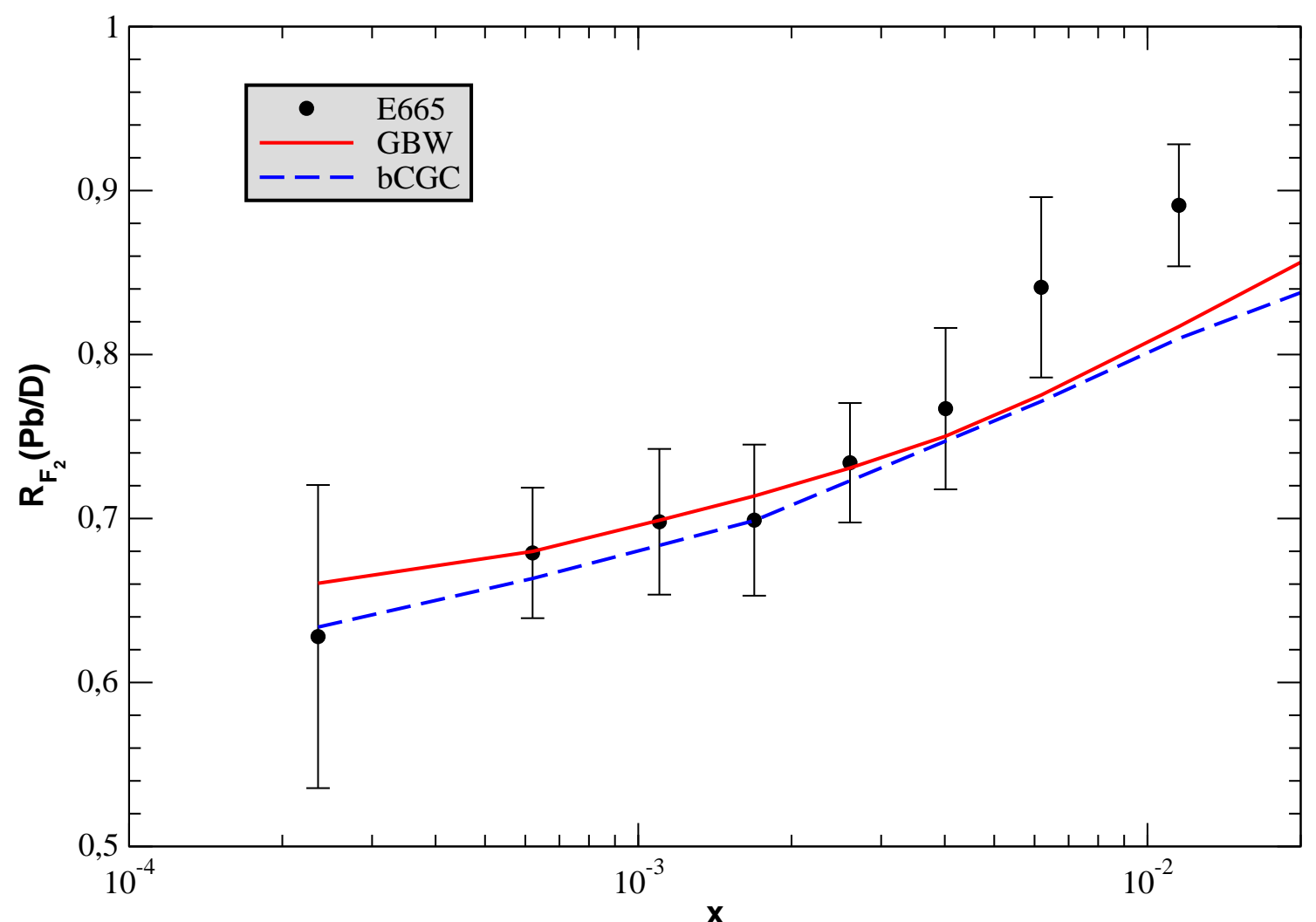

Figura 5.2: Razão $R_{F_{2}} \equiv 2 F_{2}^{A} / A F_{2}^{D}$ para $\boldsymbol{A}=\boldsymbol{P b}$. Embora unidos por uma linha os resultados dos modelos de saturação foram calculados para $\left.\left(<x>,<Q^{2}\right\rangle\right)$ dos dados da colaboração E665 [50].

$x_{0}=3,04 \times 10^{-4}$.

Em nossos cálculos nós utilizamos os dois modelos apresentados na seção 3.5, o modelo de GBW e o b-CGC. Os resultados são apresentados na próxima seção.

\subsection{Previsões lineares e não-lineares}

\subsubsection{Funções de estrutura inclusivas}

Nas figuras 5.2 e 5.3 nós comparamos com os dados da colaboração E665 [50] nossos resultados para a razão $R_{F_{2}} \equiv 2 F_{2}^{A} / A F_{2}^{D}$ obtidas usando o modelo b-CGC como entrada em nossos cálculos. Os dados de E665 mostrados nas figuras 5.2 e 5.3 envolvem não apenas valores diferentes de $x$ como também valores diferentes de $Q^{2} . F_{2}^{D}$ é a 


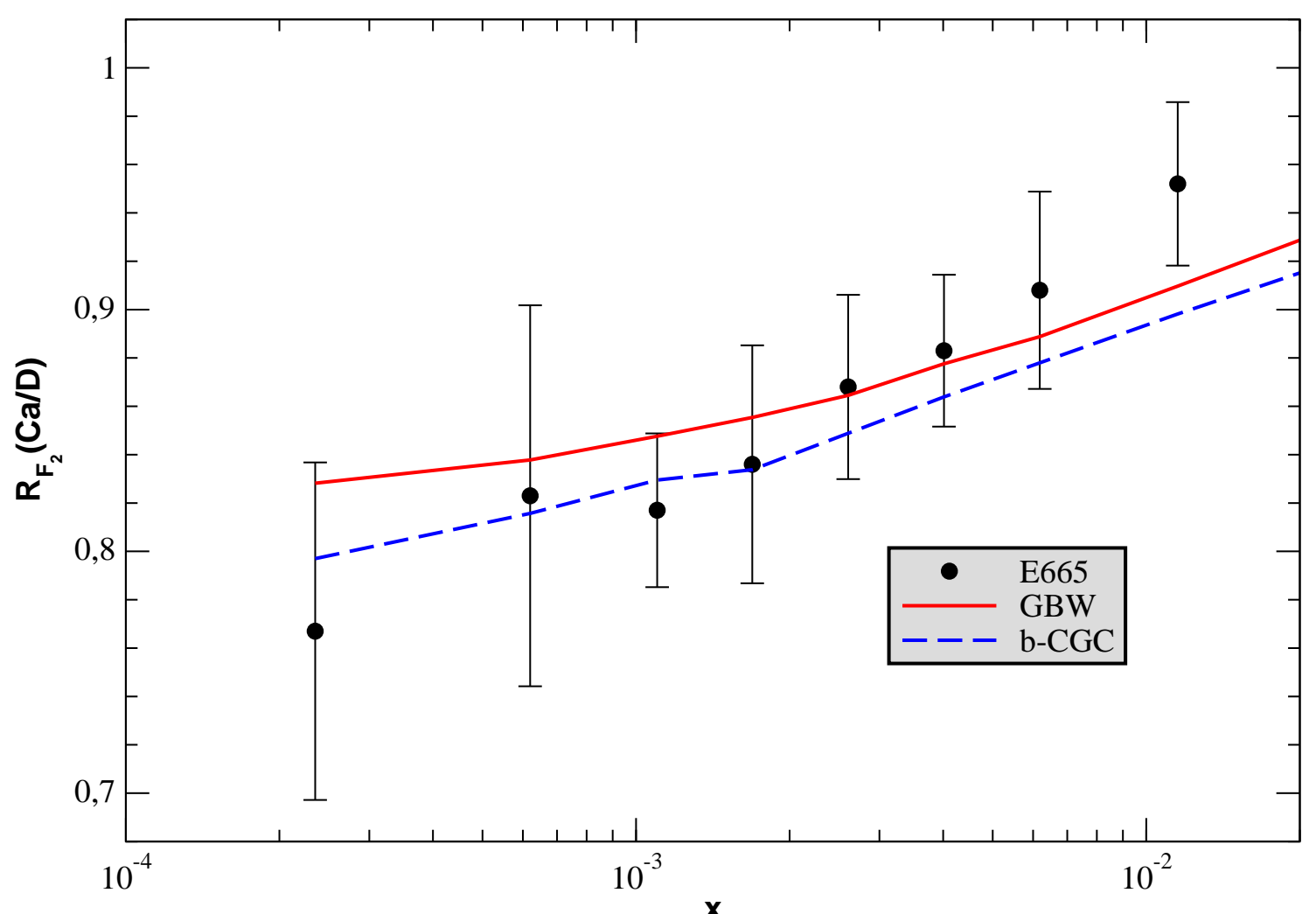

Figura 5.3: Razão $R_{F_{2}} \equiv 2 F_{2}^{A} / A F_{2}^{D}$ para $\boldsymbol{A}=$ Ca. Embora unidos por uma linha os resultados dos modelos de saturação foram calculados para $\left(<x>,<Q^{2}>\right)$ dos dados da colaboração E665 [50].

função de estrutura do dêuteron, que possui $A=2$. O chumbo $(\mathrm{Pb})$ e o Cálcio $(\mathrm{Ca})$ possuem respectivamente $A=208$ e $A=40$. Para comparação nós também mostramos as previsões obtidas usando o modelo de GBW. Ambos os modelos descrevem bem os dados experimentais na região de pequenos valores de $x\left(x \leq 10^{-2}\right)$, que é a região cinemática na qual estamos interessados. A diferença básica entre os resultados está na normalização da razão, com o modelo b-CGC apresentando um efeito nuclear maior em pequeno $x$. É importante enfatizar que depois da escolha do modelo para a seção de choque dipolo-próton nossos resultados são livres de parâmetros.

Visto que a nossa extensão (do modelo b-CGC) para o caso nuclear, através da Eq. $(5,2.6)$, descreve razoavelmente bem os dados experimentais da função de estrutura nuclear, nós podemos comparar suas previsões com aquelas obtidas usando a fatoriza- 


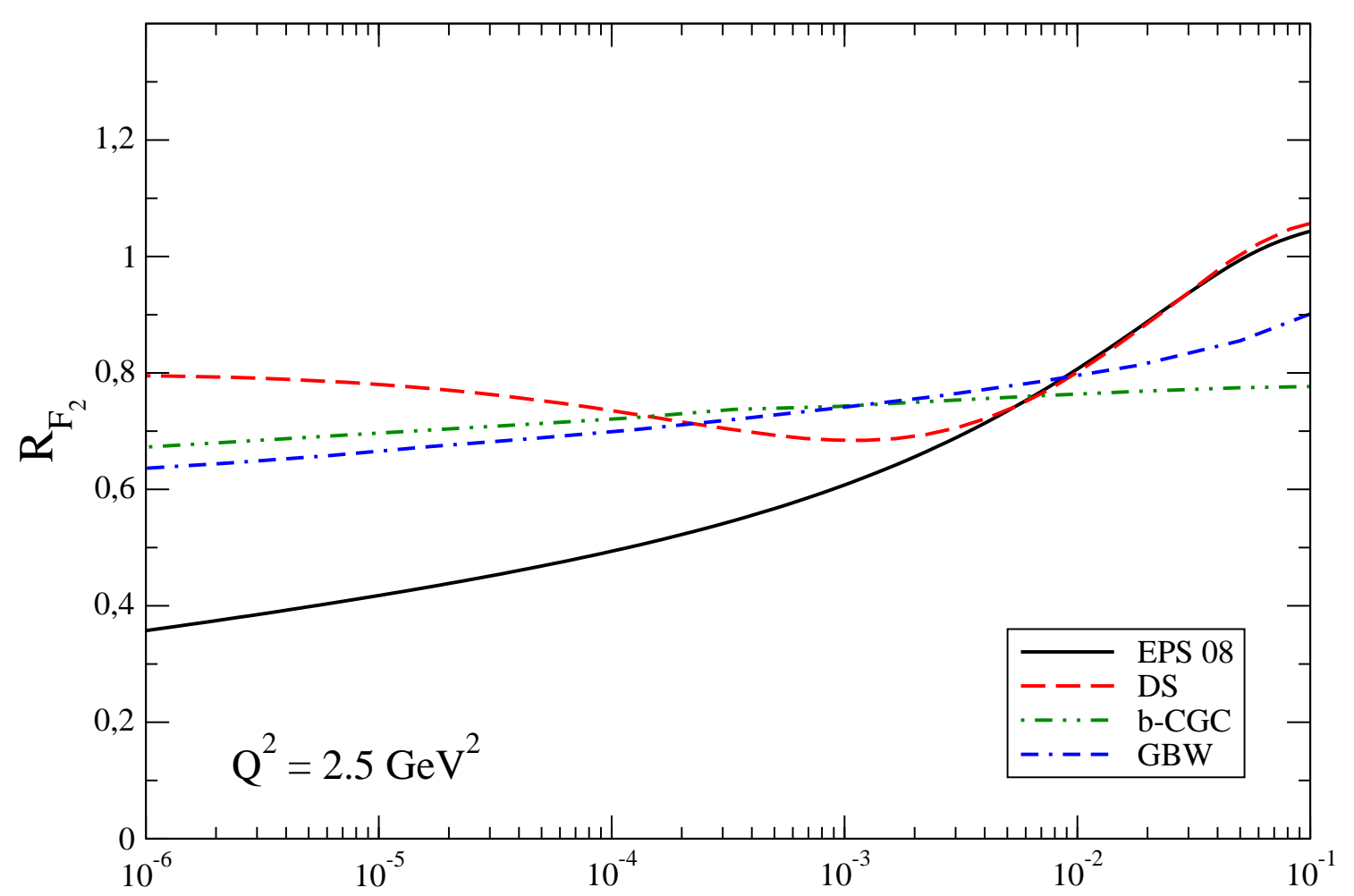

Figura 5.4: Comparação entre as previsões para $R_{F_{2}}$ dos modelos de saturação (b-CGC e GBW) e dos modelos de fatorização colinear (DS e EPS) para $Q^{2}=2,5 \mathrm{GeV}^{2}$ e $A=P b$.

ção colinear e também usá-la para calcular as funções de estrutura longitudinal e de charme.

Nas figuras 5.4 e 5.5 nós mostramos nossos resultados para a dependência em $x$ da razão $R_{F_{2}}$ para dois diferentes valores da virtualidade do fóton $Q^{2}$. Os dois modelos de saturação prevêem um comportamento semelhante na região de pequeno $x$, dando valores de $\approx 0,7$ para $x=10^{-4}$ e $Q^{2}=2,5 G e V^{2}$. Este valor cresce para $\approx 0,8$ para $Q^{2}=10 \mathrm{GeV}^{2}$. Para comparação nós também mostramos as previsões obtidas usando fatorização colinear com distribuições nucleares de pártons resultantes da análise global dos dados experimentais feita pelos grupos DS e EPS (veja seção 1.6). O motivo de escolhermos DS e EPS dentre as quatro parametrizações disponíveis é que, como podemos ver nos gráficos do capítulo 4, as curvas correspondentes representam 


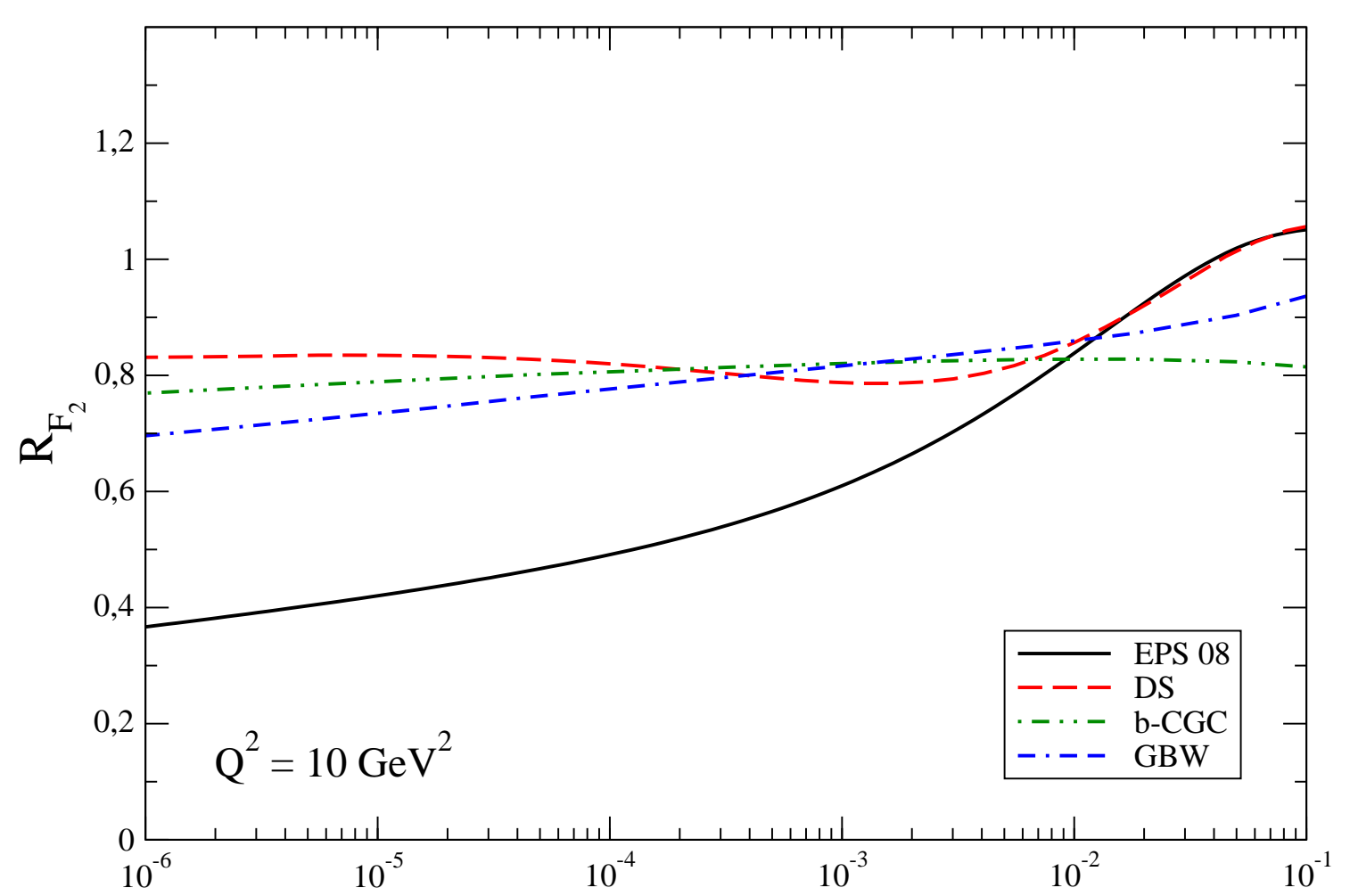

Figura 5.5: Comparação entre as previsões para $R_{F_{2}}$ dos modelos de saturação (b-CGC e GBW) e dos modelos de fatorização colinear (DS e EPS) para $Q^{2}=10 \mathrm{GeV}^{2}$ e $A=P b$.

respectivamente limites inferior e superior para a magnitude dos efeitos nucleares.

Como as previsões baseadas na fatorização colinear não incluem efeitos de saturação (elas são baseadas na dinâmica linear DGLAP), a comparação entre as previsões de saturação e colinear poderiam, em princípio, nos dizer se o observável considerado pode ser usado para discriminar entre dinâmica linear e não-linear.

Os resultados mostrados nas figuras 5.4 e 5.5 demonstram que este não é o caso da função de estrutura nuclear. Embora as previsões dos dois modelos colineares sejam semelhantes para $x$ grande $\left(\geq 10^{-2}\right)$, onde existem dados experimentais, o comportamento previsto em pequeno $x$ é muito diferente. Esta diferença é uma consequência da escolha de dados experimentais usados na análise global e das suposições relacionadas ao comportamento da densidade nuclear de glúons [1]. A diferença entre as duas 


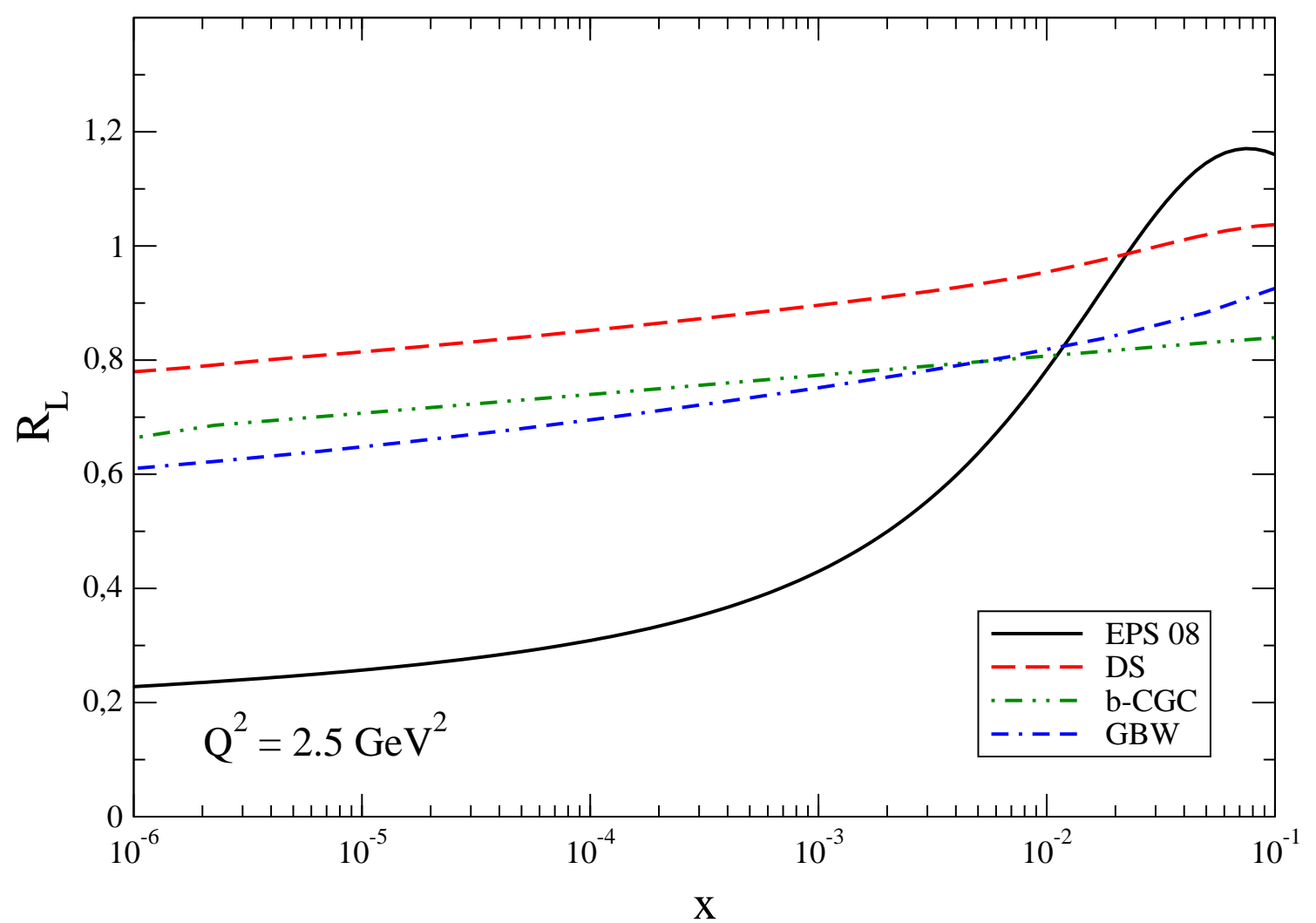

Figura 5.6: Comparação entre as previsões para $R_{L}=2 F_{L}^{A} / A F_{L}^{D}$ dos modelos de saturação (b-CGC e GBW ) e dos modelos de fatorização colinear (DS e EPS) para $Q^{2}=2,5 \mathrm{GeV}^{2}$ e $A=P b$.

previsões colineares é tão grande em pequeno $x$ que não é possível extrair qualquer informação sobre a manifestação de novos efeitos dinâmicos da QCD no estudo da função de estrutura nuclear.

Consideraremos agora o comportamento da função de estrutura longitudinal nuclear. Acredita-se que este observável pode ser usado para determinar a dinâmica da QCD em pequeno $x$ em colisões ep no HERA [51]. Um dos nossos objetivos é verificar se esta suposição é também válida em colisões $e A$.

Como podemos ver nas figuras 5.6 e 5.7, assim como no caso de $R_{F_{2}}$, as previsões para $R_{L}$ dos modelos de saturação estão contidas dentro do intervalo de incerteza dos modelos colineares. Por outro lado, a diferença entre os dois modelos colineares é maior e, neste caso (de $R_{L}$ ), os resultados para grande $x$ são também diferentes. Isto 


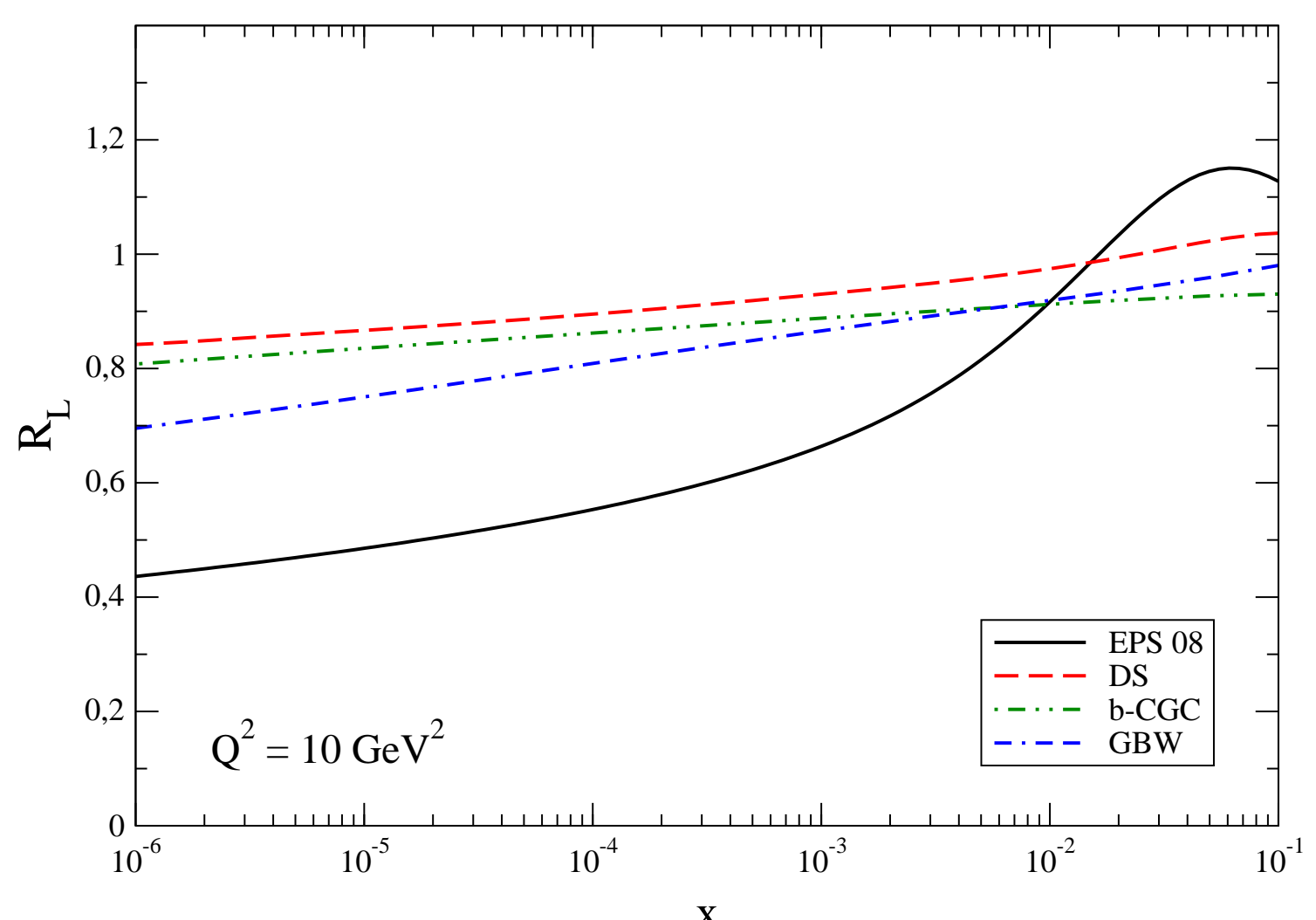

Figura 5.7: Comparação entre as previsões para $R_{L}=2 F_{L}^{A} / A F_{L}^{D}$ dos modelos de saturação (b-CGC e GBW) e dos modelos de fatorização colinear (DS e EPS) para $Q^{2}=10 \mathrm{GeV}^{2}$ e $A=P b$.

é devido à forte dependência de $F_{L}^{A}$ na distribuição nuclear de glúons, que é muito diferente nos dois modelos considerados (veja figura 1.6).

Nós consideramos também a componente de charme da função de estrutura nuclear. Nas figuras 5.8 e 5.9 vemos as previsões para $R_{C}$ dos modelos considerados. Neste caso vemos que as previsões dos modelos de saturação e do colinear DS são similares, enquanto o EPS é muito diferente. Assim como no caso de $R_{L}$ este comportamento está associado à grande magnitude dos efeitos nucleares presentes na distribuição nuclear de glúons prevista pela parametrização EPS, que tem o mais forte efeito de shadowing.

Nós não incluímos nos gráficos mostrados até aqui as previsões da parametrização EKS para $R_{F_{2}}, R_{L}$ e $R_{C}$, pois em todos os casos a curva aparece entre as curvas de GBW e EPS. Portanto podemos afirmar que, mesmo desprezando a parametrização 


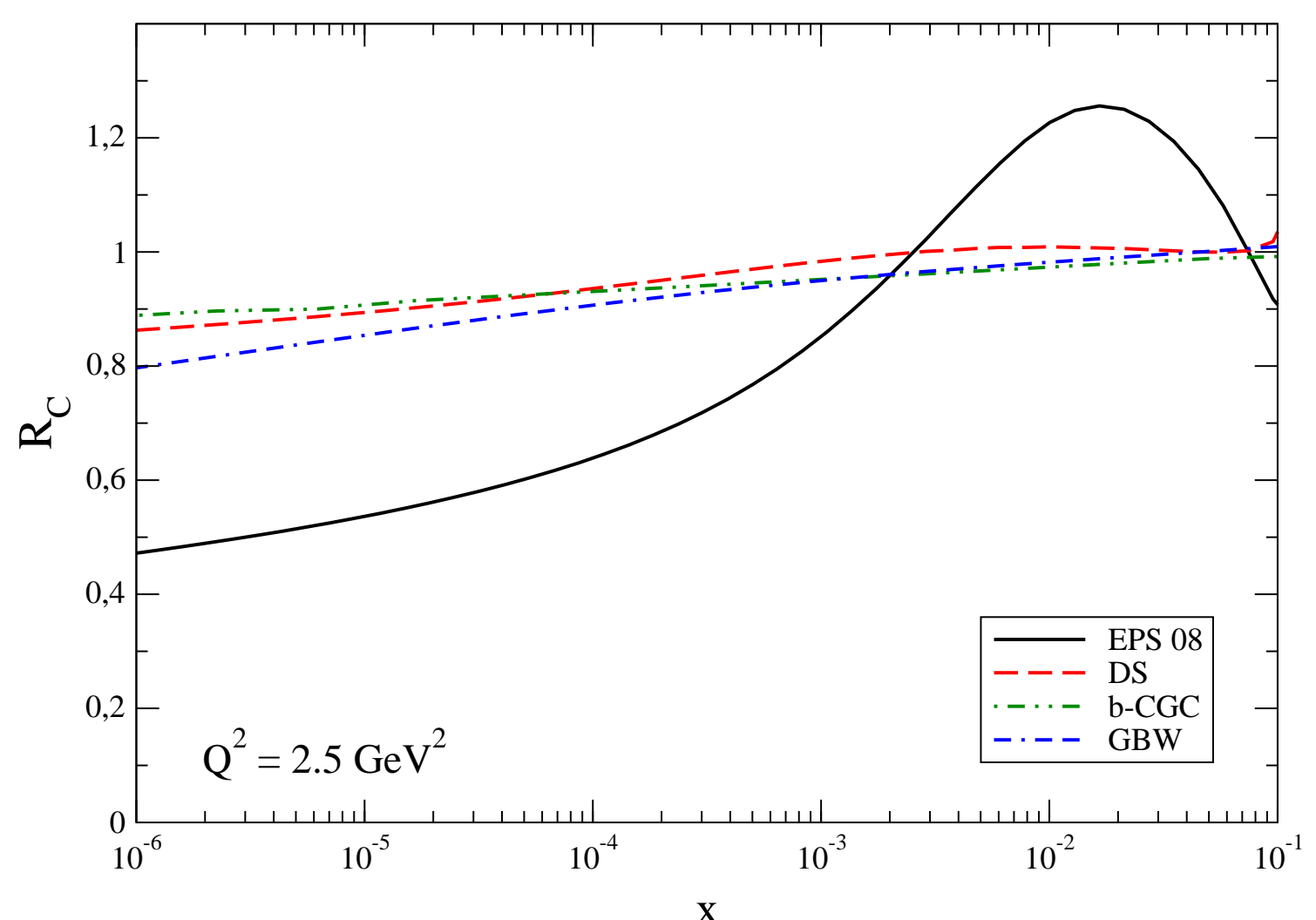

Figura 5.8: Comparação entre as previsões para $R_{C}=2 F_{2}^{c, A} / A F_{2}^{c, D}$ dos modelos de saturação (b-CGC e GBW) e dos modelos de fatorização colinear (DS e EPS) para $Q^{2}=2,5 G e V^{2}$ e $A=P b$.

EPS (que é a mais extrema), as previsões dos modelos de saturação praticamente coincidem com aquelas dos modelos colineares.

\subsubsection{Seção de choque difrativa}

A conclusão que podemos tirar dos resultados apresentados acima é que, devido à grande incerteza presente nas previsões colineares em pequeno $x$, as funções de estrutura $F_{2}, F_{2}^{c}$ e $F_{L}$ não são adequadas para a observação dos efeitos não-lineares da QCD. Para este fim, uma alternativa pode ser o estudo dos observáveis medidos em espalhamento inelástico profundo difrativo (diffractive deep inelastic scattering DDIS), visto que a seção de choque total difrativa é muito mais sensível a dipolos de grande tamanho do que os observáveis inclusivos [35, 52]. 


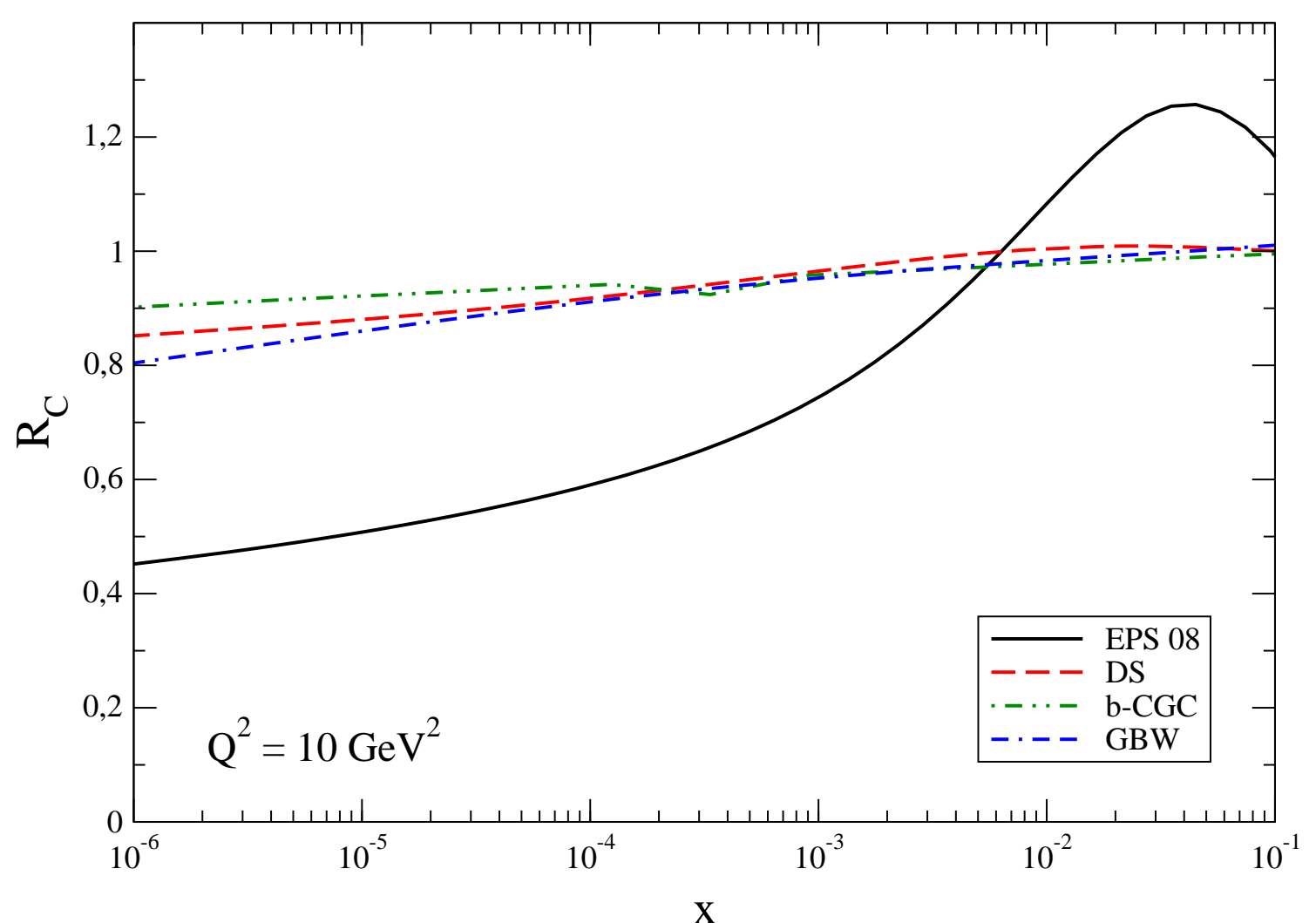

Figura 5.9: Comparação entre as previsões para $R_{C}=2 F_{2}^{c, A} / A F_{2}^{c, D}$ dos modelos de saturação (b-CGC e GBW) e dos modelos de fatorização colinear (DS e EPS) para $Q^{2}=10 \mathrm{GeV}^{2}$ e $A=P b$.

Na Ref. [52] foram calculadas quantidades observáveis como $R_{\sigma}=\sigma_{\text {diff }} / \sigma_{\text {tot }}$ (razão entre as seções de choque difrativa e total) e $F_{2}^{D(3)}$ (função de estrutura difrativa) no formalismo de dipolo usando um modelo simples de saturação. Um dos principais resultados foi o crescimento de $R_{\sigma}$ com o número de massa do alvo, especialmente na região de pequenos $x$ e $Q^{2}$, onde a razão poderia chegar a $0,3-0,4$. Uma análise detalhada dos observáveis difrativos usando o modelo b-CGC será deixada para trabalhos futuros, mas é importante verificar se a contribuição difrativa para a seção de choque total ainda é grande quando estimada usando um modelo de saturação mais realista.

Seguindo [53] nós estimamos a seção de choque difrativa total como segue: 


\section{b-CGC}

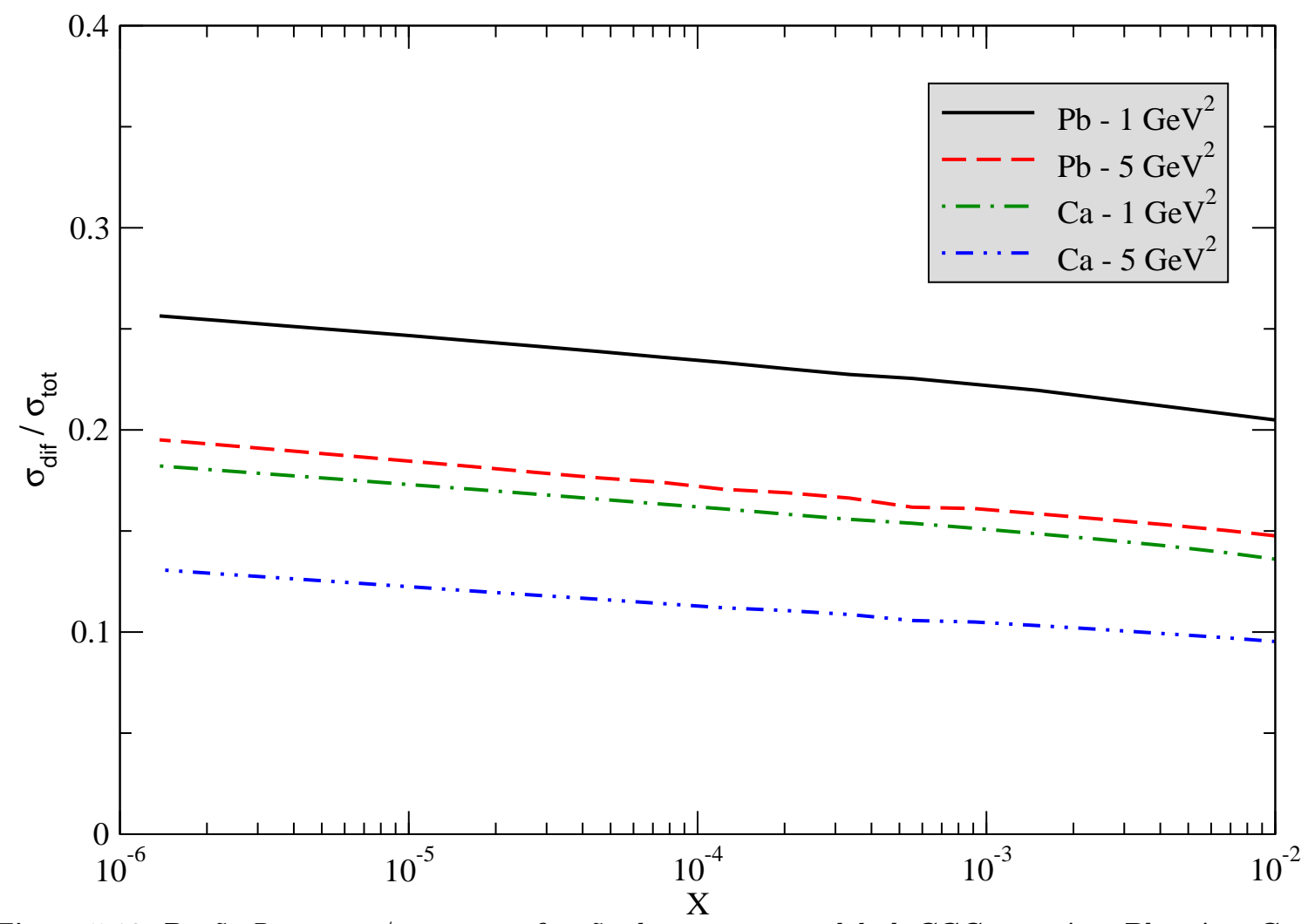

Figura 5.10: Razão $R_{\sigma}=\sigma_{\text {diff }} / \sigma_{t o t}$, como função de $x$, com o modelo b-CGC para $\boldsymbol{A}=\boldsymbol{P b}$ e $\boldsymbol{A}=\boldsymbol{C a}$ e para $Q^{2}=5 \mathrm{GeV}^{2}$ e $Q^{2}=1 \mathrm{GeV}^{2}$.

$$
\sigma_{d i f f}=\sigma_{L}^{D}+\sigma_{T}^{D} \quad ; \quad \sigma_{T, L}^{D}=\frac{1}{4} \int d z \int d^{2} r\left|\Psi_{T, L}(r, z)\right|^{2} \int d^{2} b\left(\frac{d \sigma_{d i p}^{A}}{d^{2} b}\right)^{2}
$$

onde $d \sigma_{d i p}^{A} / d^{2} b=2 \mathcal{N}^{A}(x, r, b)$, com $\mathcal{N}^{A}$ dado pela Eq. $(5,2.6)$. A expressão acima é muito semelhante à $(2,2.28)$. A diferença é que, ao invés de fazer hipóteses sobre o comportamento em $t$, a integral em $t$ é transformada numa integral no parâmetro de impacto.

Nas figuras 5.10 e 5.11 nós mostramos nossas previsões para a razão $R_{\sigma}$ como função de $x$ para dois valores de $Q^{2}$ e de $A$ considerando os modelos b-CGC e GBW. Em 


\section{GBW}

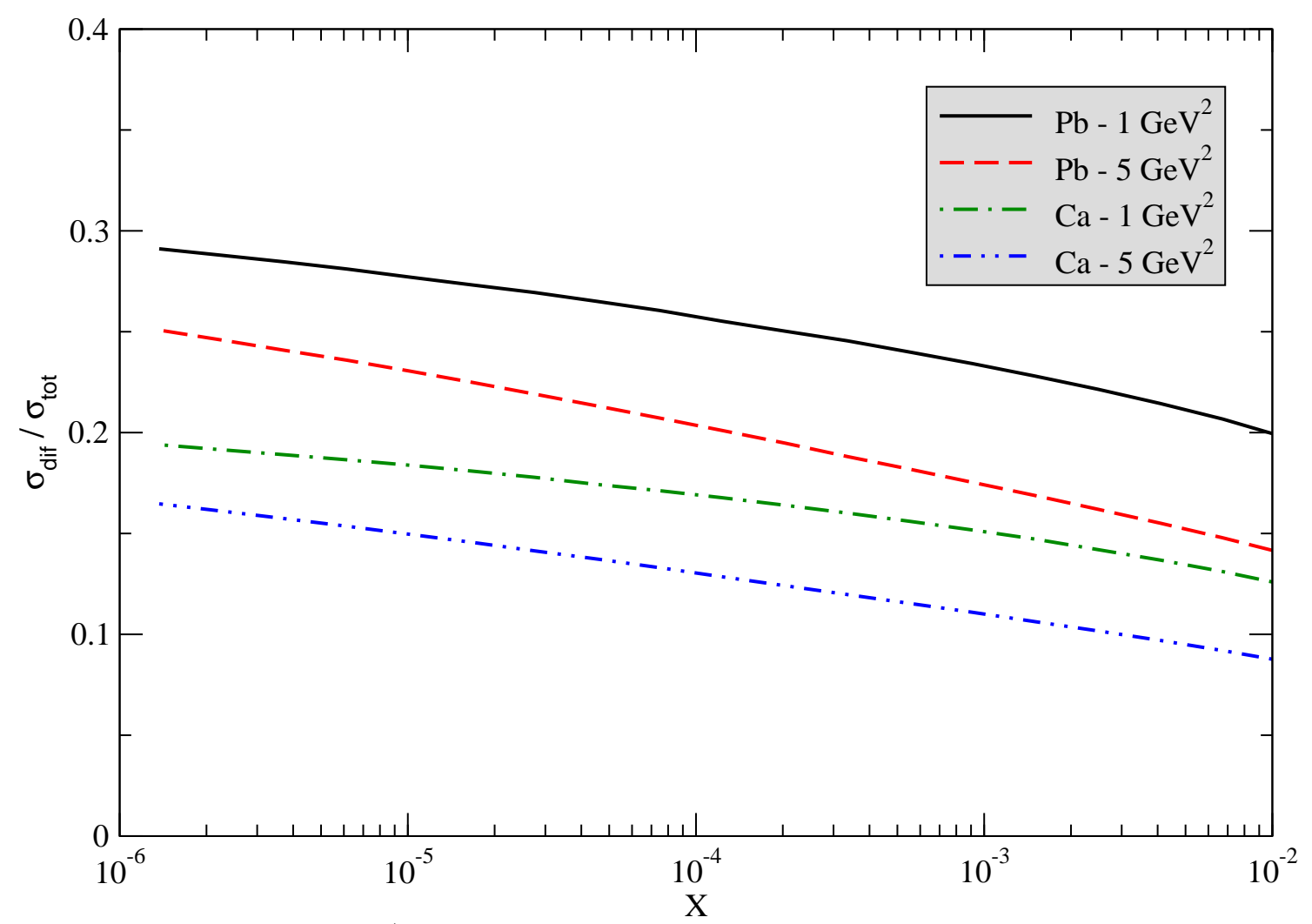

Figura 5.11: Razão $R_{\sigma}=\sigma_{d i f f} / \sigma_{t o t}$, como função de $x$, com o modelo $\boldsymbol{G B W}$ para $\boldsymbol{A}=\boldsymbol{P b}$ e $\boldsymbol{A}=\boldsymbol{C a}$ e para $Q^{2}=5 \mathrm{GeV}^{2}$ e $Q^{2}=1 \mathrm{GeV}^{2}$.

comparação com os resultados de [52], a inclusão do parâmetro de impacto no modelo de saturação reduziu a razão em cerca de $30 \%$. Entretanto, os valores previstos ainda são grandes, especialmente para pequeno $Q^{2}$ e grande $A$. Nossas previsões implicam que a razão $R_{\sigma}$ para colisões $e P b$ é um fator 2 maior do que a mesma razão observada em colisões ep no HERA. Consequentemente, o estudo de processos difrativos deverá ser uma tarefa simples em um colisor $e A$, permitindo uma análise detalhada da dinâmica da QCD.

Visto que o estudo de observáveis inclusivos não é muito promissor, o estudo de processos difrativos torna-se fundamental para determinar a dinâmica da QCD em altas energias. 


\section{Capítulo 6}

\section{Conclusão}

Nos três primeiros capítulos procuramos apresentar todos os conceitos teóricos envolvidos no desenvolvimento dos estudos que realizamos.

No capítulo 4 apresentamos nossos cálculos dos observáveis nucleares inclusivos, no formalismo da fatorização colinear, usando quatro parametrizações das distribuições nucleares de pártons disponíveis na literatura. Nós mostramos que é possível estabelecer limites para a distribuição nuclear de glúons usando estes observáveis. Isto facilitará a determinação das parametrizações que mais se aproximam da distribuição real de glúons no núcleo quando surgirem novos dados experimentais de colisões $e A$.

No capítulo 5 apresentamos nossos cálculos dos observáveis nucleares inclusivos usando modelos que levam em conta o efeito de saturação de pártons, no formalismo de dipolo de cor. Nós comparamos estes resultados com aqueles obtidos usando o formalismo de fatorização colinear e verificamos que os observáveis inclusivos não serão muito úteis para determinar se ocorre ou não a saturação. Esta conclusão foi tirada observando que os resultados obtidos usando modelos de saturação ficaram na região intermediária dos resultados obtidos com a fatorização colinear. Nós calculamos também a fração dos eventos que serão do tipo difrativo em colisões $e A$ e verificamos que ela chega a aumentar por um fator 2 em relação às colisões $e p$. Isto indica que o estudo dos eventos difrativos será uma tarefa mais fácil em um colisor $e A$, e que devemos nos concentrar nos seus observáveis para podermos verificar sinais de saturação. 


\section{Apêndice A}

\section{Detalhes da determinação da solução da equação de BFKL}

Na seção 3.3 foram apresentadas a equação de BFKL (3,3.29) e a sua solução $(3,3.30)$. Para obtermos esta solução é conveniente usarmos a transformada de Mellin com respeito à coordenada transversal [3]:

$$
N\left(r_{\perp}\right)=\int \frac{d \gamma}{2 \pi i}\left(\frac{r_{\perp}^{2}}{l^{2}}\right)^{\gamma} N(\gamma)
$$

onde $l^{2}=1 / \Lambda^{2}$ e $r_{\perp}^{2} \ll l^{2}$. Uma vez que a equação de BFKL é invariante por transformações de escala, a equação que resulta para $N(\gamma)$ é local em $\gamma$ :

$$
\frac{\partial N(\gamma)}{\partial Y}=\bar{\alpha}_{s} \chi(\gamma) N(\gamma)
$$

onde

$$
\chi(\gamma)=2 \Psi(1)-\Psi(\gamma)-\Psi(1-\gamma),
$$

sendo $\Psi$ a função di-gamma. A solução de $(0,0.2)$ é:

$$
N(\gamma)=e^{\bar{\alpha}_{s} \chi(\gamma) Y} N_{0}(\gamma)
$$


Para retornar às coordenadas espaciais, devemos calcular a integral:

$$
N=\int_{C} \frac{d \gamma}{2 \pi i} e^{\gamma r} e^{\bar{\alpha}_{s} \chi(\gamma) Y} N_{0}(\gamma)=\int_{C} \frac{d \gamma}{2 \pi i} e^{H(\gamma, r, Y)}
$$

onde $r=\ln \left(r_{\perp}^{2} / l^{2}\right)$ é negativo, pois $r_{\perp}^{2} \ll l^{2}$, e definimos $H(\gamma, r, Y) \equiv r \gamma+\bar{\alpha}_{s} \chi(\gamma) Y$. Esta integral pode ser calculada na aproximação de ponto de sela. A condição inicial $N_{0}(\gamma)$ será considerada como um valor constante, uma vez que sua contribuição para a função $H(\gamma, r, Y)$ no expoente não fica mais importante do que $r$ ou $\bar{\alpha}_{s} Y$.

O ponto de sela $\gamma_{0}$ satisfaz a equação:

$$
\left.\frac{\partial H(\gamma, r, Y)}{\partial \gamma}\right|_{\gamma_{0}}=0, \quad \gamma_{0}=\gamma_{0}(r, Y)
$$

Note que nesta equação o ponto de sela é, na verdade, função de uma única variável, ou seja, $(0,0.6)$ implica que:

$$
\begin{aligned}
& r+\left.\bar{\alpha}_{s} Y\left[-\Psi^{\prime}(\gamma)-\Psi^{\prime}(1-\gamma)\right]\right|_{\gamma_{0}}=0 \\
& \left.\frac{\partial}{\partial \gamma}[\Psi(\gamma)+\Psi(1-\gamma)]\right|_{\gamma_{0}}=\frac{r}{\bar{\alpha}_{s} Y} \equiv-\mathcal{R},
\end{aligned}
$$

$\log 0$

$$
\gamma_{0}(r, Y)=\gamma_{0}(\mathcal{R})
$$

Se estimamos a integral de Mellin apenas pelo ponto de sela, obtemos

$$
N\left(r_{\perp}\right) \simeq e^{\bar{\alpha}_{s} Y F\left(\gamma_{0}(\mathcal{R}), \mathcal{R}\right)},
$$

onde definimos $F=H /\left(\bar{\alpha}_{s} Y\right)$. O momento de saturação pode ser determinado à partir de $(0,0.8)$ usando a condição:

$$
N\left(r_{\perp}=1 / Q_{s}(Y)\right)=1
$$


o que implica que:

$$
F\left(\gamma_{0}(\mathcal{R}), \mathcal{R}\right)=0
$$

para

$$
\mathcal{R}=\mathcal{R}_{s}=-\frac{r_{s}}{\bar{\alpha}_{s} Y}=\frac{1}{\bar{\alpha}_{s} Y} \ln \frac{1}{r_{\perp, s}^{2} \Lambda^{2}}=\frac{1}{\bar{\alpha}_{s} Y} \ln \frac{Q_{s}^{2}}{\Lambda^{2}}
$$

que é uma equação para $\mathcal{R}_{s}$ e finalmente para $Q_{s}(Y)$. A solução $\mathcal{R}_{s}$ para $F\left(\gamma_{0}(\mathcal{R}), \mathcal{R}\right)=$ 0 é um número puro, $\mathcal{R}_{s} \equiv c$, e não uma função de $Y$ (veja no apêndice B).

Agora vamos reescrever a equação $(0,0.8)$ para $\mathcal{R}$ um pouco acima de $\mathcal{R}_{s}$, ou seja, para distâncias $r_{\perp}$ que, ainda sendo muito mais curtas do que o comprimento de saturação $1 / Q_{s}(Y)$, estão próximas em unidades logarítmicas. Precisamos que

$$
0<\mathcal{R}-\mathcal{R}_{s} \ll \mathcal{R}_{s}, \text { ou } 1<\ln \frac{Q^{2}}{Q_{s}^{2}(Y)} \ll \ln \frac{Q_{s}^{2}(Y)}{\Lambda^{2}}
$$

A condição $\mathcal{R} \gg \mathcal{R}_{s}$ (ou $Q^{2} \gg Q_{s}^{2}(Y)$ ) assegura que estamos no regime linear. A condição $\mathcal{R}-\mathcal{R}_{s} \ll \mathcal{R}_{s}$ permite-nos estudar a aproximação de $N\left(r_{\perp}\right)$ na direção da saturação numa expansão limitada em potências de $\mathcal{R}-\mathcal{R}_{s}$. Em ordem linear temos para essa expansão

$$
\begin{aligned}
F\left(\gamma_{0}(\mathcal{R}), \mathcal{R}\right) & \simeq F\left(\gamma_{0}\left(\mathcal{R}_{s}\right), \mathcal{R}_{s}\right)+\left.\frac{d}{d \mathcal{R}} F\left(\gamma_{0}(\mathcal{R}), \mathcal{R}\right)\right|_{\mathcal{R}=\mathcal{R}_{s}}\left(\mathcal{R}-\mathcal{R}_{s}\right)+\ldots \\
& =-\gamma_{s}\left(\mathcal{R}-\mathcal{R}_{s}\right)+\ldots
\end{aligned}
$$

Portanto, acima da escala de saturação, a amplitude de espalhamento dipolo-hádron é

$$
N\left(r_{\perp}\right) \simeq \kappa e^{-\bar{\alpha}_{s} Y \gamma_{s}\left(\mathcal{R}-\mathcal{R}_{s}\right)}
$$

Uma vez que

$$
\bar{\alpha}_{s} Y\left(\mathcal{R}-\mathcal{R}_{s}\right)=\ln \frac{1}{r_{\perp}^{2} Q_{s}^{2}(Y)}
$$

podemos escrever

$$
N\left(r_{\perp}\right) \simeq \kappa\left(r_{\perp}^{2} Q_{s}^{2}(Y)\right)^{\gamma_{s}}
$$


para $\kappa<1$. Vemos que esta equação mostra o scaling geométrico com a dimensão anômala

$$
\gamma=1-\gamma_{s}
$$

determinada pelo ponto de sela $\gamma_{s} \equiv \gamma_{0}\left(\mathcal{R}_{s}\right)$ na saturação. Portanto, dentro da região $Q_{s}^{2} \leq Q^{2} \leq Q_{s}^{4} / \Lambda^{2}$, a equação BFKL tem uma solução que prediz o scaling geométrico.

Incluindo termos de segunda ordem na expansão $(0,0.9)$, a equação para $N\left(r_{\perp}\right)$ fica [42]:

$$
N\left(r_{\perp}\right) \simeq \kappa\left(r_{\perp}^{2} Q_{s}^{2}(Y)\right)^{\gamma_{s}} \exp \left[-\frac{\gamma_{s}^{\prime}}{2 \bar{\alpha}_{s} Y}\left(\ln \frac{1}{r_{\perp}^{2} Q_{s}^{2}(Y)}\right)^{2}\right]
$$

onde definimos $\gamma_{s}^{\prime} \equiv \frac{d \gamma_{0}(\mathcal{R})}{d \mathcal{R}} \mathcal{R}_{s}$ 


\section{Apêndice B}

\section{Determinação do ponto de sela na saturação}

Apresentaremos aqui uma maneira de determinar o valor do ponto de sela na saturação tal qual discutido no Apêndice A.

Considere a equação $(0,0.7)$ satisfeita pelo ponto de sela $\gamma_{0}$. Dela podemos deduzir uma equação para um valor particular $\gamma_{s} \equiv \gamma_{0}\left(\mathcal{R}_{s}\right)$ tal que o ponto de sela na saturação fica [3]:

$$
\left.\frac{\partial}{\partial \gamma}[\Psi(\gamma)+\Psi(1-\gamma)]\right|_{\gamma_{s}}=-\mathcal{R}_{s}
$$

Multiplicando ambos os lados da equação acima por $\gamma_{s}$ temos:

$$
-\left.\gamma_{s} \frac{\partial}{\partial \gamma}[\Psi(\gamma)+\Psi(1-\gamma)]\right|_{\gamma_{s}}=\gamma_{s} \mathcal{R}_{s}
$$

onde

$$
\gamma_{s} \mathcal{R}_{s}=\gamma_{s}\left(-\frac{r_{s}}{\bar{\alpha}_{s} Y}\right)
$$

Como $\left.H\left(\gamma_{0}(\mathcal{R}), \mathcal{R}\right)\right|_{\mathcal{R}=\mathcal{R}_{s}}=\left.F\left(\gamma_{0}(\mathcal{R}), \mathcal{R}\right)\right|_{\mathcal{R}=\mathcal{R}_{s}}=0$, temos:

$$
r_{s} \gamma_{s}+\bar{\alpha}_{s} Y \chi\left(\gamma_{s}\right)=0
$$

e portanto

$$
\chi\left(\gamma_{s}\right)=-\frac{r_{s} \gamma_{s}}{\bar{\alpha}_{s} Y}
$$


Temos também

$$
\gamma_{s} \mathcal{R}_{s}=\chi\left(\gamma_{s}\right)=2 \Psi(1)-\Psi\left(\gamma_{s}\right)-\Psi\left(1-\gamma_{s}\right)
$$

e portanto:

$$
-\left.\gamma_{s} \frac{\partial}{\partial \gamma}[\Psi(\gamma)+\Psi(1-\gamma)]\right|_{\gamma_{s}}=\chi\left(\gamma_{s}\right)
$$

ou seja,

$$
\chi^{\prime}\left(\gamma_{s}\right)=\frac{\chi\left(\gamma_{s}\right)}{\gamma_{s}}
$$

Resolvendo numericamente essa equação, encontra-se [42]: $\mathcal{R}_{s} \simeq 4.88$ e

$$
\gamma_{s} \simeq 0.63
$$




\section{Referências Bibliográficas}

[1] E. R. Cazaroto, F. Carvalho, V. P. Gonçalves and F. S. Navarra, Phys. Lett B 669 (2008) 331.

[2] E. R. Cazaroto, F. Carvalho, V. P. Gonçalves and F. S. Navarra, Phys. Lett B 671 (2009) 233.

[3] M. S. Kugeratski Souza, Dipolos de cor e interações a altas energias. São Paulo: Instituto de Física da USP, 2007. Tese de Doutorado.

[4] V. P. Gonçalves, A Cromodinâmica Quântica para sistemas densos. Porto Alegre: Instituto de Física da UFRGS, 2000. Tese de Doutorado.

[5] V. Barone and E.Predazzi, High-Energy Particle Diffraction, Springer-Verlag, Berlin Heidelberg (2002).

[6] F. Halzen and A. D. Martin, Quarks and Leptons: An Introductory Course in Modern Particle Physics, John Wiley \& Sons (1984), página 184.

[7] J.D. Bjorken, Phys. Rev. 179 (1969) 1547.

[8] R.P. Feynman, Phys. Rev. Lett. 23 (1969) 1415.

[9] R.P. Feynman, Photon Hadron Interactions, W.A. Benjamin, New York, 1972.

[10] J.D. Bjorken and E.A. Paschos, Phys. Rev. 185 (1969) 1975. 
[11] E. Iancu e R. Venugopalan, hep-ph/0303204. Publicado em QGP3, Eds. R.C. Hwa and X.N.Wang, World Scientific; E. Iancu, A. Leonidov and L. McLerran, The Color Glass Condensate: An introduction, hep-ph/0202270, publicado em QCD Perspectives on Hot and Dense Matter, Eds. J. -P. Blaizot and E. Iancu, NATO Science Series, Kluwer, 2002; E. Iancu and R, Venugopalan, The Color Glass Condensate and High Energy Scattering in QCD, hep-ph/0303104; J. JalilianMarian,J. Phys. G30, 751 (2004);

[12] M. Glück, E. Reya e A. Vogt, Eur. Phys. J. C 5 (1998), 461-470.

[13] V. Guzey, arXiv:hep-ph/09074125; Abhay Deshpande, Richard Milner, Raju Venugopalan, andWerner Vogelsang, Annu. Rev. Nucl. Part. Sci. 55 (2005) 165.

[14] P. Amaudruz et al. [New Muon Collaboration], Nucl. Phys. B 441 (1995) 3; M. Arneodo et al. [New Muon Collaboration], Nucl. Phys. B 481 (1996) 3; Nucl. Phys. B 481 (1996) 23; M.R. Adams et al. [E665 Collaboration], Z. Phys. C67 (1995) 403; Phys. Rev. Lett. 68 (1992) 3266.

[15] N. Armesto, J. Phys. G: Nucl. Part. Phys. 32 (2006) R367-R393.

[16] K.J. Eskola, V.J. Kolhinen and C.A. Salgado, Eur. Phys. J. C 9 (1999) 61.

[17] D. de Florian and R. Sassot, Phys. Rev. D 69 (2004) 074028.

[18] M. Hirai, S. Kumano and T.H. Nagai, Phys. Rev. C 76 (2007) 065207.

[19] K.J. Eskola, H. Paukkunen and C.A. Salgado, arXiv:0802.0139 [hep-ph].

[20] N. Armesto, J. Phys. G 32 (2006) R367.

[21] K.J. Eskola, H. Honkanen, V.J. Kolhinen, C.A. Salgado, Phys. Lett. B 532 (2002) 222.

[22] A. Zee, F. Wilczek and S. B. Treiman, Phys. Rev. D 10, 2881 (1974).

[23] G. Altarelli, G. Martinelli. Phys. Lett B76 (1978) 89. 
[24] A.M. Cooper-Sarkar et al. Z. Phys. C39 (1988) 281.

[25] M. Gluck, E. Reya, M. Stratmann. Nucl. Phys. B422 (1994) 37.

[26] M. Gluck, E. Reya and A. Vogt. Z. Phys. C67 (1995) 433.

[27] F. E. Low, Phys. Rev. D12 (1975) 163.

[28] S. Nussinov, Phys. Rev. Lett. 34 (1975) 1286.

[29] N. N. Nikolaev and B. G. Zakharov, Z. Phys. C49, 607 (1991); Z. Phys. C53, 331 (1992); A. H. Mueller, Nucl. Phys. B415, 373 (1994); A. H. Mueller and B. Patel, Nucl. Phys. B425, 471 (1994).

[30] L.V. Gribov, E.M. Levin and M.G. Ryskin, Phys. Rep. 100, 1 (1983).

[31] J. Jalilian-Marian, A. Kovner, A. Leonidov and H. Weigert, Nucl. Phys. B504, 415 (1997); Phys. Rev. D59, 014014 (1999); J. Jalilian-Marian, A. Kovner and H. Weigert, Phys. Rev. D59, 014015 (1999); H. Weigert, Nucl. Phys. A703, 823 (2002).

[32] A. L. Ayala Filho, Formalismo de Glauber para interações partônicas. Porto Alegre: Instituto de Física da UFGRS, 1998. Tese de Doutorado.

[33] A. L. Ayala Filho, M. B. Gay Ducati and E. M. Levin, Nucl. Phys. B493, 305 (1997), ibid. B511, 355 (1998).

[34] A. L. V. Ramos dos Reis, Saturação de gluons e perda de energia em colisões hadrônicas. São Paulo, USP, 2006. Dissertação de Mestrado.

[35] K. Golec-Biernat and M. Wüsthoff, Phys. Rev. D 59, 014017 (1999), ibid. D60 114023 (1999).

[36] L. McLerran, arXiv:hep-ph/0402137. 
[37] R. C. Hwa, C. B. Yang and R. J. Fries, Phys. Rev. C71, 024902 (2005); J. w. Qiu and I. Vitev, Phys. Lett. B632, 507 (2006); B. Z. Kopeliovich, J. Nemchik, I. K. Potashnikova, M. B. Johnson and I. Schmidt, Phys.Rev. C72, 054606 (2005).

[38] E. Iancu, K. Itakura, S. Munier, Phys. Lett. B590, 199 (2004).

[39] I. I. Balitsky, Nucl. Phys. B463 (1996) 99; Y.V. Kovchegov, Phys. Rev. D 60 (1999) 034008.

[40] A. M. Stasto, Acta Phys. Polon. B 35, 3069 (2004).

[41] E. Levin and K. Tuchin, Nucl. Phys. B 573, 833 (2000).

[42] E. Iancu, K. Itakura and L. McLerran, Nucl. Phys A708, 327 (2002).

[43] I. I. Balitsky, Nucl. Phys. B463, 99 (1996), Phys. Rev. Lett. 81, 2024 (1998), Phys. Rev. D 60, 014020 (1999), Phys. Lett. B 518, 235 (2001); I.I. Balitsky and A.V. Belitsky, Nucl. Phys. B629, 290 (2002).

[44] E. Iancu, A. Leonidov and L. McLerran, Nucl.Phys. A692 (2001) 583; E. Ferreiro, E. Iancu, A. Leonidov and L. McLerran, Nucl. Phys. A701, 489 (2002).

[45] J. Jalilian-Marian, A. Kovner, L. McLerran and H. Weigert, Phys. Rev. D 55, 5414 (1997); J. Jalilian-Marian, A. Kovner and H. Weigert, Phys. Rev. D 59, 014014 (1999), ibid. 59, 014015 (1999), ibid. 59034007 (1999); A. Kovner, J. Guilherme Milhano and H. Weigert, Phys. Rev. D 62, 114005 (2000); H. Weigert, Nucl. Phys. A703, 823 (2002).

[46] H. Kowalski, L. Motyka and G. Watt, Phys. Rev. D 74, 074016 (2006).

[47] G. Watt, arXiv:0712.2670 [hep-ph].

[48] N. Armesto, Eur. Phys. J. C26, 35 (2002).

[49] V. P. Gonçalves and M. V. T. Machado, Eur. Phys. J. C 30, 387 (2003). 
[50] E665 Collaboration: M.R. Adams et al. Z. Phys. C 67, 403 (1995).

[51] V. P. Gonçalves and M. V. T. Machado, Eur. Phys. J. C 37, 299 (2004); M. V. T. Machado, Eur. Phys. J. C 47, 365 (2006).

[52] M. S. Kugeratski, V. P. Gonçalves and F. S. Navarra, Eur. Phys. J. C 46, 413 (2006).

[53] H. Kowalski, T. Lappi and R. Venugopalan, Phys. Rev. Lett. 100, 022303 (2008). 